Bull. Soc. math. France

129 (3), 2001, p. $379-448$

\title{
INVARIANT JETS OF A SMOOTH DYNAMICAL SYSTEM
}

\author{
BY SOPHIE LEMAIRE
}

\begin{abstract}
The local deformations of a submanifold under the effect of a smooth dynamical system are studied with the help of Oseledets' multiplicative ergodic theorem. Equivalence classes of submanifolds, called jets, are introduced in order to describe these local deformations. They identify submanifolds having the same approximations up to some order at a given point. For every order $k$, a condition on the Lyapunov exponents of the dynamical system is established insuring the convergence of the $k$-jet of a submanifold evolving under the action of the dynamical system. This condition can be satisfied even by stable dynamical systems. The limit is a $k$-jet which is invariant by the dynamical system.

RÉSUmÉ (Les jets invariants d'un système dynamique). - Nous étudions les déformations locales d'une sous-variété évoluant sous l'action d'un système dynamique régulier. Afin de décrire ces déformations, nous introduisons des classes d'équivalence de sous-variétés, appelées jets, qui identifient les sous-variétés ayant les mêmes approximations en un point jusqu'à un certain ordre. Pour tout entier $k$, nous obtenons une condition sur les exposants de Lyapounov du système dynamique assurant la convergence des jets d'ordre $k$ des images d'une sous-variété par le système. Cette condition n'exclut pas les systèmes dynamiques stables. La limite obtenue est un jet d'ordre $k$ invariant par le système dynamique.
\end{abstract}

Texte reçu le 15 mars 2000, accepté le 7 mars 2001

Sophie Lemaire, Laboratoire de Mathématique, Bâtiment 425, Université Paris XI, 91405 Orsay • E-mail : Sophie.Lemaire@math.u-psud.fr

2000 Mathematics Subject Classification. — 37D25, 37H15.

Key words and phrases. — Random dynamical systems, Lyapunov exponents, multiplicative ergodic theory, jets, Pesin theory. 


\section{Introduction}

One of the basic results for smooth dynamical systems is Oseledets' multiplicative ergodic theorem [11] which describes the asymptotic behaviour of a linear system. To obtain information on a smooth nonlinear dynamical system, $\left(\phi_{t}\right)_{t \in \mathbb{T}}(\mathbb{T}=\mathbb{Z}$ or $\mathbb{R})$, defined on a smooth manifold $M$, one may apply Oseledets' theorem to a linearization of the system. Under some assumptions, the theorem asserts that there exist reals $\lambda_{1}>\cdots>\lambda_{r}$ and, for almost every point $x \in M$, a splitting of the tangent space $T_{x} M$ into measurable subspaces

$$
T_{x} M=E_{1}(x) \oplus \cdots \oplus E_{r}(x),
$$

such that the nonzero vectors of a subspace $E_{i}(x)$ are expanded exponentially fast by time evolution, with $\lambda_{i}$ as asymptotic rate. The real $\lambda_{i}$ is called the $i$-th Lyapunov exponent of the dynamical system.

Pesin theory gives a nonlinear extension of Oseledets' theorem by defining a nonlinear analogue of the subspace

$$
V_{i}=E_{1} \oplus \cdots \oplus E_{i}
$$

for $i \in\{1, \ldots, r\}$ such that $\lambda_{i}>0$. It is called the unstable manifold associated with $\lambda_{i}$ and consists of the points $y$ satisfying

$$
\varlimsup_{t \rightarrow+\infty} \frac{1}{t} \log \left(d\left(\phi_{-t}(x), \phi_{-t}(y)\right)\right) \leq-\lambda_{i} .
$$

Another extension of Oseledets' theorem can be obtained by noting that this theorem gives information on the asymptotic behaviour of the tangent space of a submanifold under the effect of a dynamical system. For instance, assume that $\left(\phi_{n}\right)_{n \in \mathbb{Z}}$ is a smooth, reversible and ergodic dynamical system on $\mathbb{R}^{N}$, satisfying Oseledets' theorem. If $V$ is a subspace whose dimension equals that of $V_{s}$ for some $s \in\{1, \ldots, r\}$ and such that $V \cap \bigoplus_{i=s+1}^{r} E_{i}=\{0\}$ almost surely, then the tangent space at $x$ of the submanifold

$$
M_{n}(x)=\phi_{n}\left(V+\phi_{-n}(x)\right)
$$

converges in probability to $V_{s}(x)$, as $n$ tends to infinity.

It is therefore natural to look for the asymptotic behaviour of higher-order approximations. M. Cranston and Y. Le Jan have studied the second order approximation for isotropic Brownian flows in $\mathbb{R}^{N}$ [8], [3] and for random walks on diffeomorphisms of $\mathbb{R}^{N}[9],[2]$. Notably, they have established in [2] that the condition, $\lambda_{s+1}-2 \lambda_{s}<0$, implies the convergence in probability of the second fundamental form of $M_{n}(x)$ at $x$. Thus, the second fundamental form of $M_{n}(x)$ at $x$ may converge even if $\lambda_{s}<0$, that is when no unstable manifold is associated with $\lambda_{s}$. For example, the Lyapunov exponents of isotropic Brownian flows in $\mathbb{R}^{d}$ always satisfy the condition $\lambda_{2}-2 \lambda_{1}<0$. The aim of this paper is to extend Oseledets' theorem for higher-order approximations. By analogy to jets of maps, equivalence classes of submanifolds, also called jets,

TOME $129-2001-\mathrm{N}^{\mathrm{O}} 3$ 
are introduced in order to describe any order approximations of a submanifold at a given point. A convergence result is proved for any order jets of a submanifold evolving under the effect of the dynamical system $\left(\phi_{n}\right)_{n \in \mathbb{Z}}$. This result for the 2-jets corresponds to the one M. Cranston and Y. Le Jan have established for the second fundamental form. However, the study of the third approximation reveals the specificity of the second fundamental form. Indeed, the second fundamental form of $M_{n}$ can be written as the partial sum of a "geometric series" that converges when $\lambda_{s+1}-2 \lambda_{s}<0$. In contrast, formulas which characterize approximations of orders greater than two cannot be used directly; a convergence result can be proved only after reorganizing these formulas. The limits of the jets are in a way invariant by the dynamical system and vary continuously on sets defined by Y.B. Pesin [12] as it is the case for Oseledets' spaces $V_{i}, i=1, \ldots, r$.

These results will be proved for random dynamical systems (RDS) as they are defined by L. Arnold in [1]. RDS cover deterministic dynamical systems as well as important classes of random processes (random walks in a group of transformations [5], flows generated by a random or a stochastic differential equation [7], etc.).

The paper is organized as follows. The first Section presents the setting and the results for any order approximations: the generalization will be proved for a local RDS in $\mathbb{R}^{N}$ with a fixed point and the result for a general RDS on a manifold will follow as a consequence. The first three approximations are studied successively in Section 2, presenting the ideas and the inequalities on which the proof of the asymptotic behaviour of any order approximations is based. Finally, Section 3 gives the iterative process allowing to describe the approximations for orders greater than two and to prove the convergence results with the help of the inequalities established in Section 2.

\section{A nonlinear extension of the Oseledets' theorem}

1.1. The local random dynamical system. - Let $(\Omega, \mathcal{A}, \mathbb{P})$ be a probability space and let $\theta: \Omega \rightarrow \Omega$ be an invertible transformation such that $\mathbb{P}$ is $\theta$-ergodic. Throughout the paper, one considers a $C^{\infty}$ local $\operatorname{RDS}$ on $\mathbb{R}^{N}$ (where $N$ is an integer greater than one) over $\theta$ which fixes 0 . Such a system can be generated by a measurable mapping $\phi: D \rightarrow \mathbb{R}^{N}$ where $D$ is a measurable subset of $\Omega \times \mathbb{R}^{N}$ with the following properties: for all $\omega \in \Omega$,

(i) $D(\omega):=\left\{x \in \mathbb{R}^{N}\right.$ such that $\left.(\omega, x) \in D\right\}$ is an open neighbourhood of 0 in $\mathbb{R}^{N}$,

(ii) $\phi(\omega): D(\omega) \rightarrow \mathbb{R}^{N}$ is a $C^{\infty}$ diffeomorphism onto its image, which fixes 0 .

The set of such applications will be denoted by $\mathcal{C}$.

BULLETIN DE LA SOCIÉtÉ MATHÉMATIQUE DE FRANCE 
Applications $\phi_{n}$ for $n \in \mathbb{Z}$ are then defined by

$$
\phi_{n}= \begin{cases}\phi\left(\theta^{n-1}\right) \circ \cdots \circ \phi & \text { if } n \geq 1, \\ \operatorname{Id} & \text { if } n=0, \\ \phi^{-1}\left(\theta^{n}\right) \circ \cdots \circ \phi^{-1}\left(\theta^{-1}\right) & \text { if } n \leq-1 .\end{cases}
$$

Each map $\phi_{n}$ is well-defined on a neighbourhood of 0 in $\mathbb{R}^{N}$ and is a $C^{\infty}$ diffeomorphism from a neighbourhood $U_{n}$ of 0 onto another one.

The first derivative of $\phi(\omega)$ at 0 , denoted by $A(\omega)$, generates a linear RDS $\left(A_{n}\right)_{n \in \mathbb{Z}}$ over $\theta$, where $A_{n}(\omega)$ is the first derivative of $\phi_{n}(\omega)$ at 0 :

$$
A_{n}= \begin{cases}A\left(\theta^{n-1}\right) \cdots A & \text { if } n \geq 1 \\ \operatorname{Id} & \text { if } n=0, \\ A^{-1}\left(\theta^{n}\right) \cdots A^{-1}\left(\theta^{-1}\right)=A_{-n}\left(\theta^{n}\right)^{-1} & \text { if } n \leq-1 .\end{cases}
$$

Oseledets' theorem can be applied under the following integrability condition:

$$
\log ^{+}\left\|A^{ \pm 1}\right\| \in L^{1}(\Omega, \mathbb{P}) .
$$

It gives information about the growth rate of $\left\|A_{n} v\right\|$ for each $v \in \mathbb{R}^{N}$ :

ThEOREM 1.1 (V.I. Oseledets [11], [10]). — There exist a $\theta$-invariant set $\tilde{\Omega} \in \mathcal{A}$ of full measure, reals $\lambda_{1}>\cdots>\lambda_{r}$ and positive integers $d_{1}, \ldots, d_{r}$ with $d_{1}+\cdots+d_{r}=N$, such that for all $\omega \in \tilde{\Omega}$, there is a measurable splitting of $\mathbb{R}^{N}$ into $\mathbb{R}^{N}=E_{1}(\omega) \oplus \cdots \oplus E_{r}(\omega)$, satisfying the following properties: for all $i \in\{1, \ldots, r\}$

- $\operatorname{dim}\left(E_{i}(\omega)\right)=d_{i}$,

- $A(\omega) E_{i}(\omega)=E_{i}(\theta(\omega))$,

- $n^{-1} \log \left\|A_{n}(\omega) v\right\|$ converges to $\lambda_{i}$ uniformly in $v \in E_{i}(\omega) \cap S^{N-1}$ as $n$ tends to $+\infty$ or $-\infty$

For $i \in\{1, \ldots, r\}$, the integer $d_{i}$ is called the multiplicity of the $i$-th Lyapunov exponent $\lambda_{i}$.

1.2. A random family of submanifolds. - Fix $s \in\{1, \ldots, r\}$. Denote the subspace $\bigoplus_{i=1}^{s} E_{i}$ by $E^{s}$ and its dimension by $d$, i.e. $d=\sum_{i=1}^{s} d_{i}$. The restriction of an application $\xi \in \mathcal{C}$ to $\mathbb{R}^{d}$ defines a parametrization of a random family of $d$-dimensional submanifolds of $\mathbb{R}^{N}$ passing through 0 : for all $\omega \in \Omega$, there exists an open neighbourhood, $W(\omega)$, of 0 in $\mathbb{R}^{N}$, such that

$$
V_{n}(\omega)=\xi(\omega)\left(U_{n}(\omega) \cap W(\omega) \cap \mathbb{R}^{d}\right)
$$

is a $d$-dimensional submanifold of $\mathbb{R}^{N}$.

By applying the $\operatorname{RDS}\left(\phi_{n}\right)$ between times $-n$ and 0 , one defines a random family of $d$-dimensional submanifolds of $\mathbb{R}^{N}$ passing through 0 , denoted by $\mathcal{V}_{n}$ :

$$
\mathcal{V}_{n}:=\phi_{n}\left(\theta^{-n}\right)\left(V_{n}\left(\theta^{-n}\right)\right) \text {. }
$$

TOME $129-2001-\mathrm{N}^{\mathrm{O}} 3$ 
The aim of this paper is to study the asymptotic behaviour of the approximations of the sequence $\left(\mathcal{V}_{n}\right)_{n}$ at 0 .

\subsection{Description of any order approximations of a submanifold. -}

With the help of local parametrizations, equivalence classes of submanifolds will be introduced in order to describe all submanifolds having the same contacts up to some order at 0 (proofs of the statements are developed in the Appendix A).

1.3.1. Jets of maps. - For two Euclidean spaces $E$ and $F$, let $C_{0}^{\infty}(E, F)$ denote the class of $C^{\infty}$ maps defined on a neighbourhood of 0 in $E$ with values in $F$ and let $C_{0,0}^{\infty}(E, F)$ denote the subclass of maps in $C_{0}^{\infty}(E, F)$ that fix 0 .

Definition 1.2. - Let $k \in \mathbb{N}^{*}$. Two maps $f, g \in C_{0}^{\infty}(E, F)$ are said to "have a contact of order at least $k$ at 0 " if $f(0)=g(0)$ and if for all $j \in\{1, \ldots, k\}$, $D^{j} f(0)=D^{j} g(0)$.

This relation is an equivalence relation on $C_{0}^{\infty}(E, F)$. The equivalence class of a function $f$ at 0 is denoted by $j_{0}^{k}(f)$ and called the " $k$-jet of $f$ at 0 ". For a subclass $\mathcal{F}$ of $C_{0}^{\infty}(E, F), J_{0}^{k}(\mathcal{F})$ will be the set of $k$-jets at 0 of applications of $\mathcal{F}$. The space $J_{0}^{k}(\mathcal{F})$ is endowed with the distance $d_{k}$ defined by

$$
d_{k}\left(j_{0}^{k}(f), j_{0}^{k}(g)\right)=\max \left(\|f(0)-g(0)\|,\left\|D^{i} f(0)-D^{i} g(0)\right\|, i \in\{1, \ldots, k\}\right)
$$

for every $f, g \in \mathcal{F}$.

\subsubsection{Parametrizations}

Definition 1.3. - Let $S$ be a $d$-dimensional $C^{\infty}$ submanifold of $\mathbb{R}^{N}$, let $x$ in $\mathbb{R}^{N}$ and let $E$ be a $d$-dimensional subspace of $\mathbb{R}^{N}$. A map $f \in C_{0}^{\infty}\left(E, \mathbb{R}^{N}\right)$ is called a "parametrization of $S$ at $x$ " if

(i) $f$ is an homeomorphism from a neighbourhood $U$ of 0 in $E$ onto its image;

(ii) $\operatorname{Df}(0)$ is injective;

(iii) $f(0)=x$;

(iv) $f(U)=V \cap S$ where $V$ is a neighbourhood of 0 in $\mathbb{R}^{N}$.

The set of maps in $C_{0}^{\infty}\left(E, \mathbb{R}^{N}\right)$ satisfying properties (i) and (ii) are called embeddings and thus will be denoted by $\operatorname{Emb}_{0}^{\infty}\left(E, \mathbb{R}^{N}\right)$ and the subset of maps which also satisfy (iii) will be denoted by $\operatorname{Emb}_{0, x}^{\infty}\left(E, \mathbb{R}^{N}\right)$.

REMARK 1.4. - To shorthand the notations, $\mathbb{R}^{d}$ will denote the vector subspace $\mathbb{R}^{d} \times\{0\}^{N-d}$ of $\mathbb{R}^{N}$ for every $d \in\{1, \ldots, N\}$.

1.3.3. Contacts of a submanifold at 0 . - Using jets of parametrizations, one may define jets for smooth submanifolds of $\mathbb{R}^{N}$ :

Definition 1.5. - Let $k$ be a positive integer. Two $C^{\infty}$ submanifolds $S_{1}$ and $S_{2}$ of $\mathbb{R}^{N}$ passing through 0 have a "contact of order at least $k$ at 0 " if 
there exist $d \in\{1, \ldots, N\}$ and two parametrizations $f_{1}, f_{2} \in \operatorname{Emb}_{0,0}^{\infty}\left(\mathbb{R}^{d}, \mathbb{R}^{N}\right)$ of $S_{1}$ and $S_{2}$ at 0 such that $j_{0}^{k}\left(f_{1}\right)=j_{0}^{k}\left(f_{2}\right)$.

This relation defines an equivalence relation on the set of $C^{\infty}$ submanifolds of $\mathbb{R}^{N}$ passing through 0 (see Lemma A.5). The equivalence class of a $C^{\infty}$ submanifold $S$ will be denoted by $j_{0}^{k}(S)$ and called the " $k$-jet of $S$ at 0 ". The set of all $k$-jets at 0 of $C^{\infty}$ submanifolds of $\mathbb{R}^{N}$ will be denoted by $\mathcal{J}_{0}^{k}\left(\mathbb{R}^{N}\right)$. If $S$ is a $C^{\infty}$ submanifold of $\mathbb{R}^{N}$ passing through 0 and $h \in \operatorname{Emb}_{0,0}^{\infty}\left(\mathbb{R}^{N}, \mathbb{R}^{N}\right)$, then the $k$-jet of $h(S)$ at 0 depends uniquely on $j_{0}^{k}(h)$ and $j_{0}^{k}(S)$. Thus one can define an operator $*$ on $J_{0}^{k}\left(\operatorname{Emb}_{0,0}^{\infty}\left(\mathbb{R}^{N}, \mathbb{R}^{N}\right)\right) \times \mathcal{J}_{0}^{k}\left(\mathbb{R}^{N}\right)$ by setting

$$
j_{0}^{k}(f)_{*} j_{0}^{k}(S)=j_{0}^{k}(f(S))
$$

for any $f \in \operatorname{Emb}_{0,0}^{\infty}\left(\mathbb{R}^{N}, \mathbb{R}^{N}\right)$ and for any $C^{\infty}$ submanifold $S$ passing through 0 .

Consider now a sequence $\left(S_{n}\right)_{n}$ of $d$-dimensional $C^{\infty}$ submanifolds of $\mathbb{R}^{N}$ passing through 0 .

Definition 1.6. - The sequence of $k$-jets of $S_{n}$ at 0 is said to converge to the $k$-jet of a submanifold $S$ at 0 , if there exist a sequence of parametrizations $f_{n} \in \operatorname{Emb}_{0,0}^{\infty}\left(\mathbb{R}^{d}, \mathbb{R}^{N}\right)$ of $S_{n}$ at 0 and a parametrization $f \in \operatorname{Emb}_{0,0}^{\infty}\left(\mathbb{R}^{d}, \mathbb{R}^{N}\right)$ of $S$ at 0 such that $\left(j_{0}^{k}\left(f_{n}\right)\right)_{n}$ converges to $j_{0}^{k}(f)$.

In Section A.3, it is shown that this definition is consistent and is equivalent to the convergence in a complete metric space.

\subsection{Statements of results}

1.4.1. The asymptotic behaviour of the jets of the submanifolds $\mathcal{V}_{n}$ at 0 . The description of the asymptotic behaviour of $\left(j_{0}^{k}\left(\mathcal{V}_{n}\right)\right)_{n}$ will use an auxiliary sequence of submanifolds $\left(\mathcal{U}_{n}\right)_{n}$ tangent to $E^{s}$ at 0 and defined as follows:

$$
\forall n \in \mathbb{N}, \quad \mathcal{U}_{n}:=\phi_{n}\left(\theta^{-n}\right)\left(U_{n}\left(\theta^{-n}\right) \cap E^{s}\left(\theta^{-n}\right)\right)
$$

ThEOREM 1.7. - Let $k$ be an integer greater than one. Assume that $\log ^{+}\left\|A^{ \pm 1}\right\|$ and $\log ^{+}\left\|D^{j} \phi(0)\right\|$ for all $j \in\{2, \ldots, k\}$, lie in $L^{1}(\Omega, \mathbb{P})$.

- If $\lambda_{s+1}-k \lambda_{s}<0$, then the sequence $\left(j_{0}^{k}\left(\mathcal{U}_{n}\right)\right)_{n}$ converges $\mathbb{P}$-almost surely. The limit is a $k$-jet denoted by $\mathcal{S}^{(k)}$, which is "invariant by the $R D S$ " in the sense that $j_{0}^{k}(\phi)_{*} \mathcal{S}^{(k)}=\mathcal{S}^{(k)}(\theta) \mathbb{P}$-almost surely. A description of the limits $\mathcal{S}^{(j)}$ for $j \in\{1, \ldots, k\}$ can be obtained iteratively (see Proposition 3.8).

- If $\lambda_{s+1}-k \lambda_{s}<0$ and $D \xi(0)\left(\mathbb{R}^{d}\right) \cap \bigoplus_{i=s+1}^{r} E_{i}=\{0\} \mathbb{P}$-almost surely, then the sequence $\left(j_{0}^{k}\left(\mathcal{V}_{n}\right)\right)_{n}$ converges to $\mathcal{S}^{(k)}$ in probability.

REMARK 1.8. - When $k=1$, the assumption $\lambda_{s+1}-k \lambda_{s}<0$ is satisfied. Theorem 1.7 asserts that the tangent space $T_{0} \mathcal{V}_{n}$ converges in probability to $E^{s}$. As a consequence of Oseledets' theorem, one can get a more precise statement:

TOME $129-2001-\mathrm{N}^{\mathrm{O}} 3$ 
let $\Pi$ and $\Pi_{n}$ for every $n \in \mathbb{N}$ be the orthogonal projection onto $E^{s}$ and $T_{0} \mathcal{V}_{n}$ respectively.

- If $D \xi(0)\left(\mathbb{R}^{d}\right) \cap \bigoplus_{i=s+1}^{r} E_{i}=\{0\} \mathbb{P}$-almost surely, then for all $\epsilon>0$, the sequence $\left(\mathrm{e}^{-n\left(\lambda_{s+1}-\lambda_{s}+\epsilon\right)}\left\|\Pi_{n}-\Pi\right\|\right)_{n}$ converges to 0 in probability.

A proof of this last statement will be presented in Section 2.1. Let us note that $V \cap \bigoplus_{i=s+1}^{r} E_{i}=\{0\} \mathbb{P}$-almost surely for almost every subspace $V$ in the $d$-Grassmannian $G_{d}\left(\mathbb{R}^{N}\right)$, with respect to any diffuse probability measure on $G_{d}\left(\mathbb{R}^{N}\right)$.

REMARK 1.9. - For $k=2$, Theorem 1.7 gives the same statement as the one proposed by M. Cranston and Y. Le Jan [2]. The limit $\mathcal{S}^{(2)}$ is the set of $C^{\infty}$ submanifolds passing through 0 , whose tangent space at 0 is $E^{s}$ and whose second fundamental form at 0 is defined by the following series:

$$
\sum_{j=1}^{\infty}(\mathrm{I}-\Pi) A_{j-1}\left(\theta^{-(j-1)}\right) D^{2} \phi\left(\theta^{-j}\right)(0)\left(A_{-j} \Pi, A_{-j} \Pi\right) .
$$

REMARK 1.10. - The proof of Theorem 1.7 for any order $k$ uses the following fact: to establish the convergence of the $k$-jets of a sequence $\left(S_{n}\right)$ of submanifolds at 0 , it suffices to prove that the derivatives up to order $k$ of a particular parametrization at 0 of these submanifolds, called "the orthogonal parametrization", converge (definition and properties of orthogonal parametrizations are presented in the appendix A, Sections A.2 and A.3). The proof of the convergence of the derivatives of an orthogonal parametrization of $\mathcal{U}_{n}$ and $\mathcal{V}_{n}$ at 0 is based on an expansion of the derivatives with the help of diagrams (see Section 3). The aim of the development is to express the derivatives as a combination of terms that converge under the assumptions of Theorem 1.7. The study of the third derivative shows that such a development is not simply given by derivation, but that the terms obtained by derivation have to be rearranged.

REMARK 1.11. - The limits $\mathcal{S}^{(j)}$ for $j \in\{1, \ldots, k\}$, are invariant by the RDS since $\mathcal{U}_{n+1}=\phi\left(\theta^{-1}\right)\left(\mathcal{U}_{n}\left(\theta^{-1}\right)\right)$ for all $n \in \mathbb{N}$ and the composition of two $k$-jets defines a continuous map (Lemma A.1).

As $\theta$ preserves the measure $\mathbb{P}$, information on the asymptotic jets at 0 of the submanifolds $\mathcal{V}_{n}\left(\theta^{n}\right)=\phi_{n}\left(V_{n}\right)$ can be deduced from Theorem 1.7:

Corollary 1.12. - Let $k$ be an integer greater than one. Assume that $\log ^{+}\left\|A^{ \pm 1}\right\| \in L^{1}(\Omega, \mathbb{P})$ and that $\log ^{+}\left\|D^{j} \phi(0)\right\| \in L^{1}(\Omega, \mathbb{P})$ for all $j \in\{2, \ldots, k\}$. If $\lambda_{s+1}-k \lambda_{s}<0$ and $D \xi(0)\left(\mathbb{R}^{d}\right) \cap \bigoplus_{i=s+1}^{r} E_{i}=\{0\} \mathbb{P}$-almost surely, then the $k$-jet of $\phi_{n}\left(V_{n}\right)$ at 0 converges in law to $\mathcal{S}^{(k)}$.

BULletin DE LA SOCiÉtÉ MATHÉmATiQUe DE FRANCE 
1.4.2. Jets of the unstable manifolds. - When $\lambda_{s} \geq 0$, the assumption on the Lyapunov exponents, $\lambda_{s+1}-k \lambda_{s}<0$, is satisfied for every positive integer $k$. With the help of Pesin theory [12], [13], one may give a geometric interpretation of the limits $\mathcal{S}^{(k)}$ :

Corollary 1.13. - Assume that for all $k \in \mathbb{N}^{*}, \log ^{+}\left\|D^{k} \phi^{ \pm 1}(0)\right\|$ belongs to $L^{1}(\Omega, \mathbb{P})$. If $\lambda_{s}>0$, then $\mathcal{S}^{(k)}$ is $\mathbb{P}$-almost surely the $k$-jet at 0 of the local unstable manifolds tangent to $E^{s}$ at 0 .

Proof. - The construction of the local stable and unstable manifolds for smooth random dynamical systems, follows from Theorem 5.1 of [14]. For the unstable manifolds at 0 , the statement is the following:

Theorem 1.14 (D. Ruelle, 1979). - Assume that for all $k \in \mathbb{N}$,

$$
\log ^{+}\left\|D^{k} \phi^{ \pm 1}(0)\right\| \in L^{1}(\Omega, \mathbb{P}) .
$$

There exist a $\theta$-invariant set $\Omega^{\prime \prime} \in \mathcal{A}$ of full measure, measurable functions $\beta>\alpha>0$ and $\gamma>1$ on $\Omega^{\prime \prime}$ and a sequence $\left(\ell_{p}\right)_{p}$ of positive integers that converges to infinity such that:

(i) if $\lambda$ is a positive real distinct from the Lyapunov exponents, then for all $\omega \in \Omega^{\prime \prime}$

$\mathcal{V}^{u, \lambda}(\omega, \alpha)=\left\{y \in \bar{B}(0, \alpha(\omega)), \forall n \in \mathbb{N},\left\|\phi_{-n}(\omega)(y)\right\| \leq \beta(\omega) \mathrm{e}^{-n \lambda}\right\}$

is a $C^{\infty}$ submanifold of $\bar{B}(0, \alpha(\omega))$, tangent at 0 to the subspace

$$
V^{u, \lambda}(\omega)=\left\{v \in \mathbb{R}^{N}, \varlimsup_{n \rightarrow+\infty} \frac{1}{n} \log \left\|D_{0} \phi_{-n}(\omega) v\right\| \leq-\lambda\right\} ;
$$

(ii) if $y, z \in \mathcal{V}^{u, \lambda}(\omega, \alpha)$ then for all $n \in \mathbb{N}$,

$$
\left\|\phi_{-n}(\omega)(y)-\phi_{-n}(\omega)(z)\right\| \leq \gamma(\omega)\|y-z\| ;
$$

(iii) for all $p \in \mathbb{N}, \mathcal{V}^{u, \lambda}(\omega, \alpha) \subset \phi_{\ell_{p}}\left(\theta^{-\ell_{p}} \omega\right)\left(\mathcal{V}^{u, \lambda}\left(\theta^{-\ell_{p}}(\omega), \alpha\right)\right)$.

Let $\lambda \in] \lambda_{s+1}, \lambda_{s}$ [ be a positive real. By property (iii), the $k$-jets at 0 of the local unstable manifold $\mathcal{V}^{u, \lambda}(\omega, \alpha)$ and of the submanifolds $\phi_{\ell_{p}}\left(\theta^{-\ell_{p}} \omega\right)\left(\mathcal{V}^{u, \lambda}\left(\theta^{-\ell_{p}} \omega, \alpha\right)\right)$ coincide for every $p \in \mathbb{N}$ and $k \in \mathbb{N}^{*}$. By Theorem 1.7 , for every $k \in \mathbb{N}^{*}$, the $k$-jet of $\phi_{\ell_{p}}\left(\theta^{-\ell_{p}} \omega\right)\left(\mathcal{V}^{u, \lambda}\left(\theta^{-\ell_{p}} \omega, \alpha\right)\right)$ at 0 converges in probability to $\mathcal{S}^{(k)}(\omega)$, as $p$ tends to infinity. Therefore, for every $k \in \mathbb{N}^{*}$ the $k$-jet of $\mathcal{V}^{u, \lambda}(\omega, \alpha)$ at 0 is $\mathbb{P}$-almost surely equal to $\mathcal{S}^{(k)}(\omega)$.

1.4.3. The case of a random dynamical system on a manifold. - Let $\left(M,\langle\cdot, \cdot\rangle_{x}, x \in M\right)$ be a $C^{\infty}$ Riemannian manifold of dimension $N$. One may rewrite Theorem 1.7 in order to describe the asymptotic local deformations of a random family of submanifolds of $M$ under the action of a smooth RDS. Let $\left(\varphi_{n}\right)_{n \in \mathbb{Z}}$ be a smooth ergodic $\operatorname{RDS}$ on $M$. It is equivalent to consider a 
probability space $(\Omega, \mathcal{A}, \mathbb{P})$, an invertible transformation $\theta: \Omega \rightarrow \Omega$ such that $\mathbb{P}$ is $\theta$-ergodic, a $C^{\infty}$ diffeomorphism of $M$ denoted by $\varphi$ and to set

$$
\varphi_{n}= \begin{cases}\varphi\left(\theta^{n-1}\right) \circ \cdots \circ \varphi & \text { if } n \geq 1, \\ \operatorname{Id} & \text { if } n=0, \\ \varphi^{-1}\left(\theta^{n}\right) \circ \cdots \circ \varphi^{-1}\left(\theta^{-1}\right) & \text { if } n \leq-1\end{cases}
$$

One will assume that the $\operatorname{RDS}\left(\varphi_{n}\right)_{n \in \mathbb{Z}}$, has the following properties:

- there exists a probability measure $\mu$, on $\Omega \times M$ with marginal $\mathbb{P}$ on $\Omega$, which is invariant and ergodic for the skew product $\tilde{\theta}$ : $(\omega, x) \mapsto(\theta \omega, \varphi(\omega)(x)) ;$

- $\log ^{+}\left\|T_{x} \varphi(\omega)^{ \pm 1}\right\|_{x}$ belongs to $L^{1}(\Omega \times M, \mu)$.

Thus by Oseledets' theorem, there exist a $\tilde{\theta}$-invariant set $\tilde{\Omega}$ of full $\mu$-measure, reals $\lambda_{1}>\cdots>\lambda_{r}$ and positive integers $d_{1}, \ldots, d_{r}$ with $d_{1}+\cdots+d_{r}=N$, such that for all $(\omega, x) \in \tilde{\Omega}$, there is a measurable splitting of $T_{x} M$ into $T_{x} M=$ $E_{1}(\omega, x) \oplus \cdots \oplus E_{r}(\omega, x)$, satisfying the following properties: for all $i \in\{1, \ldots, r\}$

- $\operatorname{dim}\left(E_{i}(\omega, x)\right)=d_{i}$;

- $T_{x} \varphi(\omega) E_{i}(\omega, x)=E_{i}(\tilde{\theta}(\omega, x))$;

- $\left(n^{-1} \log \left\|T_{x} \varphi_{n}(\omega) v\right\|_{x}\right)$ converges to $\lambda_{i}$ uniformly for $v$ in $E_{i}(\omega, x)$ such that $\|v\|_{x}=1$, as $n$ tends to $+\infty$ or $-\infty$

1.4.3.1. Contacts of a submanifold of $M$. - The jets of maps between two $C^{\infty}$ manifolds $\Sigma$ and $\tilde{\Sigma}$ are defined by taking local charts:

Definition 1.15. - Let $f_{1}, f_{2}$ be $C^{\infty}$ applications mapping $\Sigma$ into $\tilde{\Sigma}$, let $x$ be a point of $\Sigma$ and let $k \in \mathbb{N}^{*}$. Assume that $f_{1}(x)=f_{2}(x)$. " $f_{1}$ and $f_{2}$ have a contact of order at least $k$ at $x$ " if for any chart $(\zeta, U)$ of $\Sigma$ at $x$ and for any $\operatorname{chart}(\tilde{\zeta}, \tilde{U})$ of $\tilde{\Sigma}$ at $f_{1}(x), j_{0}^{k}\left(\tilde{\zeta} \circ f_{1} \circ \zeta^{-1}\right)=j_{0}^{k}\left(\tilde{\zeta} \circ f_{2} \circ \zeta^{-1}\right)$.

The equivalence classes for this equivalence relation are also called the $k$-jets at $x$. One proceeds similarily to define jets of a $C^{\infty}$ submanifold of $M$ :

Definition 1.16. - Let $k \in \mathbb{N}^{*}$ and let $S$ and $\tilde{S}$ be two $C^{\infty}$ submanifolds of $M$ passing through a point $x$. " $S$ and $\tilde{S}$ have a contact of order at least $k$ at $x$ " if for every chart $(\zeta, U)$ of $M$ at $x, \zeta(S \cap U)$ and $\zeta(\tilde{S} \cap U)$ have a contact of order at least $k$ at 0 .

The relation "having a contact of order at least $k$ at $x$ " is an equivalence relation on the set of $C^{\infty}$ submanifolds of $M$ passing through $x$. The class of a submanifold $S$ of $M$ for this relation will be called "the $k$-jet at $x$ of $S$ " and denoted by $j_{x}^{k}(S)$. The set of $k$-jets of all $C^{\infty}$ submanifolds of $M$ will be denoted by $\mathcal{J}^{k}(M)$ and the set of $k$-jets at $x$ by $\mathcal{J}_{x}^{k}(M)$.

BULletin DE LA SOCiÉtÉ MATHÉmATiQUe DE FRANCE 
Actually, if there exists a chart $(\zeta, U)$ of $M$ at $x$ such that $\zeta(S \cap U)$ and $\zeta(\tilde{S} \cap U)$ have a contact of order at least $k$ at 0 , then it is also true for any other chart of $M$ at $x$ (see Lemma A.10).

One can define an operator $*$ on $J^{k}\left(\operatorname{Diff}^{\infty}(M)\right) \times \mathcal{J}^{k}(M)$ by setting

$$
j_{x}^{k}(f)_{*} j_{x}^{k}(S)=j_{f(x)}^{k}(f(S))
$$

if $f \in \operatorname{Diff}^{\infty}(M), x \in M$ and $S$ is a $C^{\infty}$ submanifold of $M$ passing through $x$.

It remains to define the convergence in $\mathcal{J}^{k}(M)$. In the case $M=\mathbb{R}^{N}$, the natural way to define the convergence is to use parametrizations:

Definition 1.17. - For every $n \in \mathbb{N}$, let $S_{n}$ be a $d$-dimensional submanifold of $\mathbb{R}^{N}$ passing through a point $x_{n}$. "The sequence $\left(j_{x_{n}}^{k}\left(S_{n}\right)\right)_{n}$ converges to the $k$ jet of a submanifold $S$ at a point $x$ " if there exists a sequence of parametrizations $f_{n} \in \operatorname{Emb}_{0, x_{n}}^{\infty}\left(\mathbb{R}^{d}, \mathbb{R}^{N}\right)$ of $S_{n}$ at $x_{n}$ and a parametrization $f \in \operatorname{Emb}_{0, x}^{\infty}\left(\mathbb{R}^{d}, \mathbb{R}^{N}\right)$ of $S$ at $x$ such that $\left(j_{0}^{k}\left(f_{n}\right)\right)_{n}$ converges to $j_{0}^{k}(f)$.

As usual this definition can be extended in the case of any manifold $M$ by introducing charts:

Definition 1.18. - Let $\left(x_{n}\right)$ be a sequence of points of $M$, let $x \in M$ and let $S_{n}$, for $n \in \mathbb{N}$, denote a $C^{\infty}$ submanifold of $M$ passing through $x_{n}$. "The $k$-jet of $S_{n}$ at $x_{n}$ is said to converge to the $k$-jet of a submanifold $S$ at $x$ as $n$ tends to $+\infty$ " if $\left(x_{n}\right)_{n}$ converges to $x$ and if, for every chart $(\zeta, U)$ of $M$ at $x$, the sequence $\left(j_{\zeta\left(x_{n}\right)}^{k}\left(\zeta\left(S_{n} \cap U\right)\right)\right)_{n}$ converges to $j_{0}^{k}(\zeta(S \cap U))$.

Actually, it suffices to verify the criteria for one chart to prove the convergence (see Lemma A.13).

1.4.3.2. Existence of the invariant jets. - Let $\mathcal{C}(M)$ denote the set of random parametrizations of $M$, that is the set of measurable maps $\zeta: \tilde{D} \rightarrow M$ where $\tilde{D}$ is a measurable subset of $\Omega \times M \times \mathbb{R}^{N}$ such that, for all $(\omega, x) \in \Omega \times M$ :

- $\tilde{D}(\omega, x):=\left\{z \in \mathbb{R}^{N},(\omega, x, z) \in \tilde{D}\right\}$ is an open neighbourhood of 0 in $\mathbb{R}^{N}$;

- $\zeta(\omega, x): \tilde{D}(\omega, x) \mapsto M$ is a $C^{\infty}$ diffeomorphism onto its image satisfying $\zeta(\omega, x)(0)=x$.

Without loss of generality, one may consider a random parametrization $\zeta \in \mathcal{C}(M)$ of $M$ such that for all $(\omega, x) \in \Omega \times M, D \zeta(\omega, x)(0)$ is an isometry from the Euclidean space $\left(\mathbb{R}^{N},\langle\cdot, \cdot\rangle\right)$, onto $\left(T_{x} M,\langle\cdot, \cdot\rangle_{x}\right)$. The subset of $\mathcal{C}(M)$, which consists of applications having this property, will be denoted by $\mathcal{C}_{1}(M)$.

Let $\xi \in \mathcal{C}(M)$. For every $n \in \mathbb{N}^{*}$, there exists a neighbourhood $U_{n}$ of 0 in $\mathbb{R}^{N}$, such that $\varphi_{n} \circ \xi$ is a $C^{\infty}$ diffeomorphism from $U_{n}$ onto its image. For $(\omega, x) \in \Omega \times M, \xi(\omega, x)\left(U_{n}(\omega, x) \cap \mathbb{R}^{d}\right)$ is a $C^{\infty} d$-dimensional submanifold of 
$M$ passing through $x$. Applying the $\operatorname{RDS}\left(\varphi_{n}\right)$ between times $-n$ and 0 , gives a $d$-dimensional submanifold of $M$ passing through $x$, denoted by $\mathcal{V}_{n, \xi}(\omega, x)$ :

$$
\mathcal{V}_{n, \xi}(\omega, x):=\varphi_{n}\left(\theta^{-n} \omega\right) \circ \xi\left(\tilde{\theta}^{-n}(\omega, x)\right)\left(U_{n}\left(\tilde{\theta}^{-n}(\omega, x)\right) \cap \mathbb{R}^{d}\right) .
$$

For $\zeta \in \mathcal{C}(M)$, the map $\Phi_{\zeta}$, defined by $\zeta^{-1}(\tilde{\theta}) \circ \varphi \circ \zeta$, belongs to $\mathcal{C}$ and generates a local $\operatorname{RDS}\left(\Phi_{n, \zeta}\right)_{n \in \mathbb{Z}}$ over $\tilde{\theta}$. Its Lyapunov exponents are $\lambda_{1}, \ldots, \lambda_{r}$ and Oseledets' spaces denoted by $E_{\zeta, i}, i \in\{1, \ldots, r\}$ satisfy the equalities $E_{\zeta, i}=D_{0} \zeta\left(E_{i}\right)$ for all $i \in\{1, \ldots, r\}$. Applying Theorem 1.7 to the probability space $(\Omega \times M, \mathcal{A} \otimes \mathcal{B}(M), \mu)$, endowed with the transformation $\tilde{\theta}$, and to the local $\operatorname{RDS}\left(\Phi_{n, \zeta}\right)_{n \in \mathbb{Z}}$, gives asymptotic results for the jets of the submanifolds $\mathcal{V}_{n, \xi}(\omega, x)$ at $x:$

Corollary 1.19. - Let $k \in \mathbb{N}^{*}$ and let $\xi \in \mathcal{C}(M)$. Assume that

$$
\log ^{+}\left\|T_{x} \varphi(\omega)^{ \pm 1}\right\| \in L^{1}(\Omega \times M, \mathcal{A} \otimes \mathcal{B}(M), \mu)
$$

and that there exists $\zeta \in \mathcal{C}_{1}(M)$ such that $\log ^{+}\left\|D^{j} \Phi_{\zeta}(0)\right\| \in L^{1}(\Omega \times M, \mu)$ for all $j \in\{2, \ldots, k\}$.

If $\lambda_{s+1}-k \lambda_{s}<0$ and $D \xi(0)\left(\mathbb{R}^{d}\right) \cap \bigoplus_{i=s+1}^{r} E_{i}=\{0\} \mu$-almost surely, then the sequence $\left(j_{x}^{k}\left(\mathcal{V}_{n, \xi}(\omega, x)\right)\right)_{n}$ converges in probability. The limit denoted by $\mathcal{S}^{(k)}$ does not depend on $\xi$ and is invariant by the RDS i.e. for $\mu$-almost every $(\omega, x) \in \Omega \times M$,

$$
j_{x}^{k}(\varphi(\omega)) * \mathcal{S}^{(k)}(\omega, x)=\mathcal{S}^{(k)}(\theta(\omega), \varphi(\omega)(x)) .
$$

1.4.3.3. Continuity of the invariant jets. - Let us introduce the subsets defined by Pesin [12] on which the Oseledets' subspaces are continuous. For $\epsilon>0$, $R>0$ and $\omega \in \Omega$, let $\Lambda_{R, \epsilon}(\omega)$ denote the set of points $x$ in $M$ satisfying the following properties:

- $(\omega, x) \in \tilde{\Omega}$;

- for every $n \in \mathbb{Z}, m \in \mathbb{Z}, i \in\{1, \ldots, r\}$ and $v \in E_{i}\left(\tilde{\theta}^{m}(\omega, x)\right)$;

$$
\begin{aligned}
& \left\|T \varphi_{n}\left(\tilde{\theta}^{m}(\omega, x)\right) v\right\|_{\varphi_{m+n}(\omega)(x)} \leq R \mathrm{e}^{n \lambda_{i}+(|n|+|m|) \epsilon}\|v\|_{\varphi_{m}(\omega)(x)} ; \\
& \left\|T \varphi_{n}\left(\tilde{\theta}^{m}(\omega, x)\right) v\right\|_{\varphi_{m+n}(\omega)(x)} \geq R^{-1} \mathrm{e}^{n \lambda_{i}-(|n|+|m|) \epsilon}\|v\|_{\varphi_{m}(\omega)(x)} ;
\end{aligned}
$$

- if $I$ and $J$ are two nonempty disjoint subsets of $\{1, \ldots, r\}$ then, for every $m \in \mathbb{Z}$, the sinus of the angle $\gamma\left(E_{I}\left(\tilde{\theta}^{m}(\omega, x)\right), E_{J}\left(\tilde{\theta}^{m}(\omega, x)\right)\right)$ between the vector spaces

$$
E_{I}\left(\tilde{\theta}^{m}(\omega, x)\right)=\bigoplus_{i \in I} E_{i}\left(\tilde{\theta}^{m}(\omega, x)\right) \text { and } E_{J}\left(\tilde{\theta}^{m}(\omega, x)\right)=\bigoplus_{i \in J} E_{i}\left(\tilde{\theta}^{m}(\omega, x)\right)
$$

is greater than $R^{-1} \mathrm{e}^{-|m| \epsilon}$. 
Let us note that for $\mu$-almost every $(\omega, x) \in \Omega \times M$, there exists $R \in \mathbb{N}^{*}$ such that $x \in \Lambda_{R, \epsilon}(\omega)$. These subsets are not invariant by the $\operatorname{RDS}\left(\varphi_{n}\right)_{n}$ but have the following properties:

Proposition 1.20. - Let $0<\epsilon<\frac{1}{4} \min \left(\lambda_{i}-\lambda_{i+1}, i \in\{1, \ldots, r\}\right)$ and $R>0$. For every $\omega \in \Omega$,

- $\Lambda_{R, \epsilon}(\omega)$ is a closed subset of $M$;

- there exists a real $T>R$ such that $\varphi(\omega)\left(\Lambda_{R, \epsilon}(\omega)\right) \subset \Lambda_{T, \epsilon}(\theta(\omega))$;

- The union $\Lambda_{\epsilon}(\omega)=\bigcup_{R>0} \Lambda_{R, \epsilon}(\omega)$ is invariant by the RDS, i.e. $\varphi(\omega)\left(\Lambda_{\epsilon}(\omega)\right)=\Lambda_{\epsilon}(\theta(\omega)) ;$

- $x \mapsto E_{i}(\omega, x)$ is continuous in the subset $\Lambda_{R, \epsilon}(\omega)$.

The proof of Proposition 1.20 is the same as Pesin's proof of Theorem 1.3.1 in [12]. A continuity result for the invariant $k$-jet $\mathcal{S}^{(k)}(\omega,$.$) on \Lambda_{R, \epsilon}(\omega)$ can be deduced by taking a particular parametrization $\zeta \in \mathcal{C}_{1}(M)$; $\zeta$ will be the composition of the $C^{\infty}$ exponential map associated to the $C^{\infty}$ Riemannian metric of $M$ (denoted by Exp) and a global trivialization of the tangent bundle $T M$ which is isometric on the fibers. Before stating the continuity result, let us introduce a notation: $\Phi$ will denote the map $\varphi$ lifted by the exponential map to $T M$; for $(\omega, x) \in \Omega \times M$,

$$
\Phi(\omega, x):=\operatorname{Exp}_{\varphi(\omega)(x)}^{-1} \circ \varphi(\omega) \circ \operatorname{Exp}_{x}
$$

(there exists a measurable positive function $\delta$ such that, for each $(\omega, x) \in \Omega \times M$, $\Phi(\omega, x)$ is well-defined on the $\delta(\omega, x)$-open ball of $T_{x} M$ centered at 0 and is a $C^{\infty}$ diffeomorphism onto its image).

COROllary 1.21. - Let us assume that

$\int_{\Omega \times M} \log ^{+}\left\|\left(T_{x} \varphi(\omega)\right)^{ \pm 1}\right\| \mathrm{d} \mu(\omega, x)$ and $\int_{\Omega} \log ^{+} \sup _{x \in M}\left\|D^{j} \Phi(\omega, x)(0)\right\| \mathrm{d} \mathbb{P}(\omega)$, are finite for every $j \in\{2, \ldots, k\}$. Let $s \in\{1, \ldots, r\}$ and let $d$ denote the dimension of the subspace $E^{s}:=\bigoplus_{i=1}^{s} E_{i}$.

If $\lambda_{s+1}-k \lambda_{s}<0$, then there exists a random $k$-jet of $d$-dimensional submanifolds of $M$ at $x$ denoted by $\mathcal{S}^{(k)}(\omega, x)$ such that:

- $\mathcal{S}^{(k)}$ is invariant by the $\operatorname{RDS}\left(\varphi_{n}\right)_{n \in \mathbb{Z}}$;

- For $\mathbb{P}$-almost every $\omega \in \Omega, x \mapsto \mathcal{S}^{(k)}(\omega, x)$ is continuous in $\Lambda_{R, \epsilon}(\omega), R$ and $\epsilon$ being positive numbers such that

$$
\epsilon< \begin{cases}\min \left(\frac{1}{20} \lambda_{s}, \frac{1}{4}\left(\lambda_{i}-\lambda_{i+1}\right), i \in\{1, \ldots, r-1\}\right) & \text { if } \lambda_{s}>0 \\ \min \left(\frac{1}{20 k}\left(k \lambda_{s}-\lambda_{s+1}\right), \frac{1}{4}\left(\lambda_{i}-\lambda_{i+1}\right), i \in\{1, \ldots, r-1\}\right) & \text { if } \lambda_{s} \leq 0\end{cases}
$$

The proof of Corollary 1.21 is postponed until Appendix B because it will take up some details of the proof of Theorem 1.7.

TOME $129-2001-\mathrm{N}^{\mathrm{O}} 3$ 


\section{The first three approximations}

2.1. Convergence of the tangent space of $\mathcal{V}_{\boldsymbol{n}}$ at $\mathbf{0}$ toward $\boldsymbol{E}^{\boldsymbol{s}}$. - Let $\Pi$ and $\Pi_{n}$ denote the orthogonal projections onto $E^{s}$ and $T_{0} \mathcal{V}_{n}$ respectively. The purpose of the subsection is to establish a lower bound for the convergence speed of $\Pi_{n}$ to $\Pi$ as $n$ tends to $+\infty$ (Proposition 2.1). This lower bound will be useful in the study of the asymptotic behaviour of any order approximations of $\mathcal{V}_{n}$ at 0 .

Proposition 2.1. - Let $\epsilon>0$. If $D \xi(0)\left(\mathbb{R}^{d}\right) \cap \bigoplus_{i=s+1}^{r} E_{i}=\{0\} \mathbb{P}$-almost surely, then the sequence

$$
\left(\max _{k, \ell \in\{0, \ldots, n\}} \mathrm{e}^{-n\left(\lambda_{s+1}-\lambda_{s}+\epsilon\right)-k\left(\lambda_{s}+\epsilon\right)-\ell\left(-\lambda_{s+1}+\epsilon\right)}\left\|A_{-\ell}\left(\Pi_{n}-\Pi\right) A_{k}\left(\theta^{-k}\right)\right\|\right)_{n}
$$

converges in probability to zero.

The proof of Proposition 2.1 uses two consequences of Oseledets' theorem. First:

Lemma 2.2. - Let $i \in\{1, \ldots, r\}$. The sequence $\left(n^{-1} \log \left\|A_{n}\left(\theta^{-n}\right)_{\mid E_{i}\left(\theta^{-n}\right)}\right\|\right)_{n}$ converges $\mathbb{P}$-almost surely to $\lambda_{i}$.

Proof. - The sequence $\left(\log \left\|A_{n}\left(\theta^{-n}\right)_{\mid E_{i}\left(\theta^{-n}\right)}\right\|\right)_{n}$ is subadditive since $A_{n}\left(\theta^{-n}\right) E_{i}\left(\theta^{-n}\right)=E_{i}$ for all $i \in\{1, \ldots, r\}$ and $n \in \mathbb{N}$. Thus, one may apply Kingman's subadditive ergodic Theorem [6]:

Theorem 2.3 (J.F. Kingman, 1968). — Let $(X, \mathcal{A}, m)$ be a probability space, let $T: X \rightarrow X$ be a measurable transformation such that $m$ is $T$-invariant and let $\left(f_{n}\right)_{n \geq 1}$ be a sequence of measurable functions from $X$ to $\mathbb{R} \cup\{-\infty\}$ such that

(i) $f_{1}^{+} \in L^{1}(X, \mathcal{A}, m)$;

(ii) $\left(f_{n}\right)_{n \geq 1}$ is subadditive, i.e. for all $n, m \geq 1, f_{n+m} \leq f_{n}+f_{m}\left(T^{n}\right)$. fying:

There exists a measurable function, $f: X \rightarrow \mathbb{R} \cup\{-\infty\}$, T-invariant satis-

- $f^{+} \in L^{1}(X, m)$;

- $\lim _{n \rightarrow+\infty} n^{-1} f_{n}=f$ m-almost surely;

- $\lim _{n \rightarrow+\infty} n^{-1} \int f_{n}(x) \mathrm{d} m(x)=\inf _{n} n^{-1} \int f_{n}(x) \mathrm{d} m(x)=\int f(x) \mathrm{d} m(x)$.

The sequence $\left(n^{-1} \log \left\|A_{n}\left(\theta^{-n}\right)_{\mid E_{i}\left(\theta^{-n}\right)}\right\|\right)_{n \geq 1}$ has a $\mathbb{P}$-almost surely limit as $n$ tends to $+\infty$. Since $\mathbb{P}$ is $\theta$-invariant, its limit is almost surely equal to the limit of the sequence $\left(n^{-1} \log \left\|A_{n \mid E_{i}}\right\|\right)$, i.e. $\lambda_{i}$.

The second result required states that the angle between two subspaces $E_{i}$ and $E_{j}$ in Oseledets' splitting does not decrease exponentially fast to zero (see for example, [1] for a proof):

BULletin DE LA SOCIÉtÉ MATHÉMATIQUE DE FRANCE 
Lemma 2.4. - For every $i \in\{1, \ldots, r\}$, let $\pi_{i}$ denote the projection onto $E_{i}$ along $\bigoplus_{j \neq i} E_{j}$. Then

$$
\lim _{n \rightarrow+\infty} \frac{1}{n} \log \left\|\pi_{i}\left(\theta^{-n}\right)\right\|=0 \quad \mathbb{P} \text {-almost surely. }
$$

Let us now return to the convergence of the tangent space of $\mathcal{V}_{n}$ at 0 to $E^{s}$ and prove a preliminary result:

Lemma 2.5. - Let $\epsilon>0$.

1) Let $k \in\{1, \ldots, s\}$. The sequence $\left(\mathrm{e}^{-n\left(\lambda_{s+1}-\lambda_{k}+\epsilon\right)}\left\|\left(\Pi_{n}-\Pi\right)_{\mid E_{k}}\right\|\right)_{n}$ converges to zero in probability.

2) The sequence $\left(\mathrm{e}^{-n\left(\lambda_{s+1}-\lambda_{s}+\epsilon\right)}\left\|\Pi_{n}-\Pi\right\|\right)_{n}$ converges to zero in probability.

3) Let $\ell \in\{s+1, \ldots, d\}$ and $k \in\{1, \ldots, d\}$. The sequence

$$
\left(\mathrm{e}^{-n\left(\lambda_{\ell}-\max \left(\lambda_{k}, \lambda_{s}\right)+\epsilon\right)}\left\|\pi_{\ell}\left(\Pi_{n}-\Pi\right)_{\mid E_{k}}\right\|\right)_{n}
$$

converges to zero in probability.

Proof. — To prove Lemma 2.5, fix a random basis $\left(u_{1}, \ldots, u_{d}\right)$ of $D \xi(0)\left(\mathbb{R}^{d}\right)$. The following notations will be used:

- for a random vector $v$ and $n \in \mathbb{N}$, set $v^{(n)}=A_{n}\left(\theta^{-n}\right) v\left(\theta^{-n}\right)$;

- for $d$ random vectors $\left(v_{1}, \ldots, v_{d}\right)$, for any $i \in\{1, \ldots, d\}$ set

$$
\underline{v}=v_{1} \wedge \cdots \wedge v_{d} \quad \text { and } \quad \underline{v}^{\wedge i}=v_{1} \wedge \cdots \wedge v_{i-1} \wedge v_{i+1} \wedge \cdots \wedge v_{d} .
$$

1) Let $k \in\{1, \ldots, s\}$ and let $e(\omega)$ be a unit vector of $\mathrm{E}_{k}(\omega)$. It follows from the equality $\left(\Pi-\Pi_{n}\right) e=\left(\mathrm{I}-\Pi_{n}\right) e$ that

$$
\left\|e \wedge \underline{u}^{(n)}\right\|=\left\|\left(\mathrm{I}-\Pi_{n}\right) e \wedge \underline{u}^{(n)}\right\|=\left\|\left(\mathrm{I}-\Pi_{n}\right) e\right\| \cdot\left\|\underline{u}^{(n)}\right\| .
$$

First let us bound $\left\|e \wedge \underline{u}^{(n)}\right\|$. For a family of indexes $\left(i_{1}, \ldots, i_{d}\right) \in\{1, \ldots, r\}^{d}$, the $p$-vector $e \wedge\left(\pi_{i_{1}} \wedge \cdots \wedge \pi_{i_{d}}\right)\left(\underline{u}^{(n)}\right)$ is equal to zero except when, for every $j \in\{1, \ldots, r\}$, the number $q_{j}$ of projections in a subspace $E_{j}$ is smaller than $d_{j}$ (the dimension of $E_{j}$ ) and when the number of projections on the subspace $E_{k}$ is smaller than $d_{k}-1$. If these two conditions are satisfied, then

$$
\sum_{j=1}^{r} q_{j} \lambda_{j} \leq \sum_{i=1}^{s} d_{i} \lambda_{i}+\lambda_{s+1}-\lambda_{k}
$$

and

$$
\begin{aligned}
\| e & \wedge\left(\pi_{i_{1}} \wedge \cdots \wedge \pi_{i_{d}}\right)\left(\underline{u}^{(n)}\right) \| \\
& =\left\|e \wedge \wedge^{d} A_{n}\left(\theta^{-n}\right)\left(\pi_{i_{1}}\left(\theta^{-n}\right) \wedge \cdots \wedge \pi_{i_{d}}\left(\theta^{-n}\right)\right)\left(\underline{u}\left(\theta^{-n}\right)\right)\right\| \\
& \leq\left\|\wedge^{d} A_{n}\left(\theta^{-n}\right)_{\mid\left(E_{i_{1}} \wedge \cdots \wedge E_{i_{d}}\right)\left(\theta^{-n}\right)}\right\| \cdot\left\|\pi_{i_{1}}\left(\theta^{-n}\right)\right\| \cdots\left\|\pi_{i_{d}}\left(\theta^{-n}\right)\right\| .
\end{aligned}
$$

TOME $129-2001-\mathrm{N}^{\mathrm{O}} 3$ 
By Oseledets' theorem, for all $\epsilon>0$, there exists a $\mathbb{P}$-almost surely finite random variable $C_{\epsilon}$, such that for all $n \in \mathbb{N}$,

$$
\left\|\wedge^{d} A_{n}\left(\theta^{-n}\right)_{\mid\left(E_{i_{1}} \wedge \cdots \wedge E_{i_{d}}\right)\left(\theta^{-n}\right)}\right\| \leq C_{\epsilon} \mathrm{e}^{n\left(\sum_{j=1}^{r} q_{j} \lambda_{j}+\epsilon\right)} .
$$

Moreover, for all $i \in\{1, \ldots, r\}$,

$$
\lim _{n \rightarrow+\infty} \frac{1}{n} \log \left(\left\|\pi_{i}\left(\theta^{-n}\right)\right\|\right)=0
$$

$\mathbb{P}$-almost surely. Thus, for all $\epsilon>0$, there exists a $\mathbb{P}$-almost surely finite random variable $C_{\epsilon}$, such that for all $n \in \mathbb{N}$,

$$
\left\|e \wedge \underline{u}^{(n)}\right\| \leq C_{\epsilon} \exp \left(n\left(\sum_{j=1}^{s} d_{j} \lambda_{j}+\lambda_{s+1}-\lambda_{k}+\epsilon\right)\right) .
$$

Consider now $\left\|\underline{u}^{(n)}\right\|^{-1}$. Let $\pi^{s}$ denote the projection onto $E^{s}$ along $\bigoplus_{i=s+1}^{r} E_{i}$. As the dimension of $\wedge^{d} E^{s}$ is equal to 1 ,

$$
\begin{aligned}
\left\|\underline{u}^{(n)}\right\|^{-1} & =\left\|\wedge^{d} A_{n}\left(\theta^{-n}\right) \underline{u}\left(\theta^{-n}\right)\right\|^{-1} \\
& \leq\left\|\wedge^{d} \pi^{s}\right\| \cdot\left\|\wedge^{d} A_{n}\left(\theta^{-n}\right) \wedge^{d} \pi^{s}\left(\theta^{-n}\right)\left(\underline{u}\left(\theta^{-n}\right)\right)\right\|^{-1} \\
& \leq\left\|\wedge^{d} \pi^{s}\right\| \cdot\left\|\wedge^{d} A_{n}\left(\theta^{-n}\right) \mid \wedge^{d} E^{s}\left(\theta^{-n}\right)\right\|^{-1} \cdot\left\|\wedge^{d} \pi^{s}\left(\theta^{-n}\right)\left(\underline{u}\left(\theta^{-n}\right)\right)\right\|^{-1} .
\end{aligned}
$$

By Oseledets' theorem,

$$
\lim _{n \rightarrow+\infty} \frac{1}{n} \log \left\|\wedge^{d} A_{n}\left(\theta^{-n}\right)_{\mid \wedge^{d} E^{s}\left(\theta^{-n}\right)}\right\|=\sum_{j=1}^{s} d_{j} \lambda_{j}
$$

$\mathbb{P}$-almost surely. Thus, for all $\epsilon>0$, there exists a $\mathbb{P}$-almost surely finite random variable $C_{\epsilon}$ such that for all $n \in \mathbb{N}$,

$$
\left\|\underline{u}^{(n)}\right\|^{-1} \leq C_{\epsilon} \mathrm{e}^{n\left(-\sum_{i=1}^{s} \lambda_{i} d_{i}+\epsilon\right)} \cdot\left\|\wedge^{d} \pi^{s}\left(\theta^{-n}\right) \underline{u}\left(\theta^{-n}\right)\right\|^{-1} .
$$

By assumption, $D \xi(0)\left(\mathbb{R}^{d}\right) \cap \bigoplus_{i=s+1}^{r} E_{i}=\{0\} \mathbb{P}$-almost surely. Thus $\wedge^{d} \pi^{s}(\underline{u}) \neq 0 \mathbb{P}$-almost surely and the sequence $\left(\mathrm{e}^{-n \epsilon}\left\|\wedge^{d} \pi^{s}\left(\theta^{-n}\right) \underline{u}\left(\theta^{-n}\right)\right\|^{-1}\right)$ converges to zero in probability. Therefore for all $\epsilon>0$, there exists a sequence of random variables $\left(C_{\epsilon, n}\right)_{n}$ that converges to zero in probability, such that for all $n \in \mathbb{N}$,

$$
\left\|\underline{u}^{(n)}\right\|^{-1} \leq C_{\epsilon, n} \mathrm{e}^{n\left(-\sum_{i=1}^{s} \lambda_{i} d_{i}+\epsilon\right)} .
$$

In conclusion, for all $\epsilon>0$ there exists a sequence of random variables $\left(C_{\epsilon, n}\right)_{n}$ that converges to zero in probability, such that for all $n \in \mathbb{N}$,

$$
\left\|\left(\Pi-\Pi_{n}\right)_{\mid E_{k}}\right\| \leq C_{\epsilon, n} \mathrm{e}^{n\left(\lambda_{s+1}-\lambda_{k}+3 \epsilon\right)} .
$$

2) Fix $0<\epsilon<\lambda_{s}-\lambda_{s+1}$ and $0<\delta<1$. For $n \in \mathbb{N}$, set

$$
\mathcal{A}_{n, \delta}=\left\{\omega \in \Omega \text { such that } \mathrm{e}^{-n\left(\lambda_{s+1}-\lambda_{s}+\epsilon\right)}\left\|\left(\mathrm{I}-\Pi_{n}\right) \Pi\right\|>\delta\right\} .
$$

BULLETIN DE LA SOCIÉTÉ MATHÉMATIQUE DE FRANCE 
The first point of Lemma 2.5 states that $\mathbb{P}\left(\mathcal{A}_{n, \delta}\right)$ converges to zero as $n$ tends to $+\infty$. On the other hand, Theorem A.4 established by T. Kato implies that if $\omega \notin \mathcal{A}_{n, \delta}$, then

$$
\left\|\Pi_{n}(\omega)-\Pi(\omega)\right\|=\left\|\left(\mathrm{I}-\Pi_{n}(\omega)\right) \Pi(\omega)\right\| \leq \delta \mathrm{e}^{n\left(\lambda_{s+1}-\lambda_{s}+\epsilon\right)} .
$$

Thus, the sequence $\left(\left\|\Pi_{n}-\Pi\right\| \mathrm{e}^{-n\left(\lambda_{s+1}-\lambda_{s}+\epsilon\right)}\right)_{n}$ converges to zero in probability.

3) Let $\ell \in\{s+1, \ldots, d\}, k \in\{1, \ldots, d\}$ and let $e$ be a unit vector of $E_{k}$. Then $\pi_{\ell}\left(\Pi_{n}-\Pi\right)(e)$ is equal to $\pi_{\ell} \Pi_{n}(e)$. The decomposition of the vector $\Pi_{n}(e)$ on the basis $\left(u_{i}^{(n)}\right)_{1 \leq i \leq d}$ gives:

$$
\Pi_{n}(e)=\sum_{i=1}^{d}(-1)^{i+1} u_{i}^{(n)}\left\langle e \wedge \underline{u}^{(n) \wedge i}, \underline{u}^{(n)}\right\rangle \cdot\left\|\underline{u}^{(n)}\right\|^{-2} .
$$

By Oseledets' theorem, for every $\epsilon>0$, there exists a $\mathbb{P}$-almost surely finite random variable $C_{\epsilon}$, such that for all $n \in \mathbb{N}$,

$$
\left\|\pi_{\ell}\left(u_{i}^{(n)}\right)\right\|=\left\|A_{n}\left(\theta^{-n}\right) \pi_{\ell}\left(\theta^{-n}\right)\left(u_{i}\left(\theta^{-n}\right)\right)\right\| \leq C_{\epsilon} \mathrm{e}^{n\left(\lambda_{\ell}+\epsilon\right)} .
$$

A proof similar to the one used for upper bound (3) shows that

$$
\left\|e \wedge \underline{u}^{(n) \wedge i}\right\| \leq C_{\epsilon} \exp \left(n\left(\sum_{j=1}^{s} \lambda_{j} d_{j}-\max \left(\lambda_{k}, \lambda_{s}\right)+\epsilon\right)\right) .
$$

It follows from inequalities (4) and (5) that for all $\epsilon>0$, there exists a sequence of random variables $\left(C_{\epsilon, n}\right)_{n}$ which converges in probability to zero, such that for all $n \in \mathbb{N},\left\|\pi_{\ell} \Pi_{n}(e)\right\| \leq C_{\epsilon, n} \mathrm{e}^{n\left(\lambda_{\ell}-\max \left(\lambda_{k}, \lambda_{s}\right)+\epsilon\right)}$.

Let us derive Proposition 2.1 from Lemma 2.5:

Proof of Proposition 2.1. - Let $k, \ell \in\{1, \ldots, n\}$. It suffices to decompose $A_{-\ell}\left(\Pi_{n}-\Pi\right) A_{k}\left(\theta^{-k}\right)$ using the projectors $\pi_{1}, \ldots, \pi_{r}$ and to apply Lemmas 2.4 , 2.5 and Oseledets' theorem to each term of the decomposition:

- If $i, j \in\{1, \ldots, s\}$, then

$$
\begin{aligned}
& \left\|A_{-\ell} \pi_{i}\left(\Pi_{n}-\Pi\right) \pi_{j} A_{k}\left(\theta^{-k}\right)\right\| \\
& \quad \leq\left\|A_{-\ell \mid E_{i}}\right\| \cdot\left\|\pi_{i}\right\| \cdot\left\|\left(\Pi_{n}-\Pi\right)_{\mid E_{j}}\right\| \cdot\left\|A_{k}\left(\theta^{-k}\right)_{\mid E_{j}\left(\theta^{-k}\right)}\right\| \cdot\left\|\pi_{j}\left(\theta^{-k}\right)\right\| \\
& \quad \leq C_{\epsilon, n} \mathrm{e}^{\ell\left(-\lambda_{i}+\epsilon\right)+n\left(\lambda_{s+1}-\lambda_{j}+\epsilon\right)+k\left(\lambda_{j}+\epsilon\right)}
\end{aligned}
$$

It follows from $i, j \leq s$ and $k \leq n$ that $\lambda_{j}(k-n) \leq \lambda_{s}(k-n)$ and $-\lambda_{i} \leq-\lambda_{s}$. Thus

$$
\left\|A_{-\ell} \pi_{i}\left(\Pi_{n}-\Pi\right) \pi_{j} A_{k}\left(\theta^{-k}\right)\right\| \leq C_{\epsilon, n} \mathrm{e}^{\ell\left(-\lambda_{s}+\epsilon\right)+n\left(\lambda_{s+1}-\lambda_{s}+\epsilon\right)+k\left(\lambda_{s}+\epsilon\right)} .
$$

TOME $129-2001-\mathrm{N}^{\mathrm{O}} 3$ 
- If $i \in\{1, \ldots, s\}$ and $j \in\{s+1, \ldots, r\}$, then

$$
\begin{aligned}
& \left\|A_{-\ell} \pi_{i}\left(\Pi_{n}-\Pi\right) \pi_{j} A_{k}\left(\theta^{-k}\right)\right\| \\
& \quad \leq\left\|A_{-\ell \mid E_{i}}\right\| \cdot\left\|\pi_{i}\right\| \cdot\left\|\Pi_{n}-\Pi\right\| \cdot\left\|A_{k}\left(\theta^{-k}\right)_{\mid E_{j}\left(\theta^{-k}\right)}\right\| \cdot\left\|\pi_{j}\left(\theta^{-k}\right)\right\| \\
& \quad \leq C_{\epsilon, n} \mathrm{e}^{\ell\left(-\lambda_{i}+\epsilon\right)+n\left(\lambda_{s+1}-\lambda_{s}+\epsilon\right)+k\left(\lambda_{j}+\epsilon\right)} \\
& \quad \leq C_{\epsilon, n} \mathrm{e}^{\ell\left(-\lambda_{s}+\epsilon\right)+n\left(\lambda_{s+1}-\lambda_{s}+\epsilon\right)+k\left(\lambda_{s+1}+\epsilon\right)} .
\end{aligned}
$$

- If $i \in\{s+1, \ldots, r\}$ and $j \in\{1, \ldots, r\}$, then

$$
\begin{aligned}
& \left\|A_{-\ell} \pi_{i}\left(\Pi_{n}-\Pi\right) \pi_{j} A_{k}\left(\theta^{-k}\right)\right\| \\
& \quad \leq\left\|A_{-\ell \mid E_{i}}\right\| \cdot\left\|\pi_{i}\left(\Pi_{n}-\Pi\right)_{\mid E_{j}}\right\| \cdot\left\|A_{k}\left(\theta^{-k}\right)_{\mid E_{j}\left(\theta^{-k}\right)}\right\| \cdot\left\|\pi_{j}\left(\theta^{-k}\right)\right\| \\
& \quad \leq C_{\epsilon, n} \mathrm{e}^{\ell\left(-\lambda_{i}+\epsilon\right)+n\left(\lambda_{i}-\max \left(\lambda_{j}, \lambda_{s}\right)+\epsilon\right)+k\left(\lambda_{j}+\epsilon\right)} .
\end{aligned}
$$

As $\ell, k \leq n,(n-\ell) \lambda_{i} \leq(n-\ell) \lambda_{s+1}$ and

$\triangleright$ if $j \leq s$, then $-n \max \left(\lambda_{j}, \lambda_{s}\right)+\lambda_{j} k=(k-n) \lambda_{j} \leq(k-n) \lambda_{s}$.

$\triangleright$ if $j \geq s+1$, then $-n \max \left(\lambda_{j}, \lambda_{s}\right)+\lambda_{j} k \leq-n \lambda_{s}+k \lambda_{s+1}$.

Thus

$$
\left\|A_{-\ell} \pi_{i}\left(\Pi_{n}-\Pi\right) \pi_{j} A_{k}\left(\theta^{-k}\right)\right\| \leq C_{\epsilon, n} \mathrm{e}^{\ell\left(-\lambda_{s+1}+\epsilon\right)+n\left(\lambda_{s+1}-\lambda_{s}+\epsilon\right)+k\left(\lambda_{s}+\epsilon\right)} .
$$

Therefore, for all $n \in \mathbb{N}$ and $k, \ell \in\{0, \ldots, n\}$,

$$
\left\|A_{-\ell}\left(\Pi_{n}-\Pi\right) A_{k}\left(\theta^{-k}\right)\right\| \leq C_{\epsilon, n} \mathrm{e}^{\ell\left(-\lambda_{s+1}+\epsilon\right)+n\left(\lambda_{s+1}-\lambda_{s}+\epsilon\right)+k\left(\lambda_{s}+\epsilon\right)} .
$$

2.2. Asymptotic behaviour of the second order approximation. This section presents a proof of the announced results for the second order approximation of $\mathcal{V}_{n}$ at 0 . Let

- $v_{n}$ denote the restriction of $\phi_{n}\left(\theta^{-n}\right)$ to $E^{s}\left(\theta^{-n}\right) \cap U_{n}\left(\theta^{-n}\right)$ and

- $\gamma_{n}$ denote the restriction of $\phi_{n}\left(\theta^{-n}\right) \circ \xi\left(\theta^{-n}\right)$ to $W\left(\theta^{-n}\right) \cap U_{n}\left(\theta^{-n}\right)$.

The orthogonal normalizations of $v_{n}$ and $\gamma_{n}$ at 0 designate the parametrizations of $\mathcal{U}_{n}$ and $\mathcal{V}_{n}$ at 0 defined in a neighbourhood of 0 by

$$
\Upsilon_{n}:=v_{n} \circ\left(\Pi \circ v_{n}\right)^{-1} \circ \Pi \text { and } \Gamma_{n}:=\gamma_{n} \circ\left(\Pi_{n} \circ \gamma_{n}\right)^{-1} \circ \Pi_{n} .
$$

According to Lemma A.9, a sufficient condition for the $k$-jets of $\mathcal{U}_{n}\left(\mathcal{V}_{n}\right.$ resp.) to converge at 0 is that the sequence $\left(D^{j} \Upsilon_{n}(0)\right)_{n}\left(\left(D^{j} \Gamma_{n}(0)\right)_{n}\right.$ resp. $)$ converges for all $j \in\{1, \ldots, k\}$. Thus, to prove the convergence results for the 2 -jets of $\mathcal{U}_{n}$ and $\mathcal{V}_{n}$ at 0 , it remains to establish the following proposition:

Proposition 2.6. - Assume that $\log ^{+}\left(\left\|A^{ \pm 1}\right\|\right)$ and $\log ^{+}\left(\left\|D^{2} \phi(0)\right\|\right)$ belong to $L^{1}(\Omega, \mathcal{A}, \mathbb{P})$.

- If $\lambda_{s+1}-2 \lambda_{s}<0$, then $\left(D^{2} \Upsilon_{n}(0)\right)_{n}$ converges $\mathbb{P}$-almost surely to

$$
S^{(2)}=\sum_{k=1}^{\infty}(\mathrm{I}-\Pi) A_{k-1}\left(\theta^{-(k-1)}\right) D^{2} \phi\left(\theta^{-k}\right)\left(A_{-k} \Pi, A_{-k} \Pi\right) .
$$

BULletin DE LA SOCIÉtÉ MATHÉmATIQUE DE FRANCE 
- If $\lambda_{s+1}-2 \lambda_{s}<0$ and $D \xi(0)\left(\mathbb{R}^{d}\right) \cap \bigoplus_{i=s+1}^{r} E_{i}=\{0\} \mathbb{P}$-almost surely, then $\left(D^{2} \Gamma_{n}(0)\right)_{n}$ converges in probability to $S^{(2)}$.

The following proof of Proposition 2.6 is an adaptation of M. Cranston and Y. Le Jan's proof [2] to the general case of random dynamical systems. It proceeds in three steps:

- computation of an expression of the second order derivatives of $\Upsilon_{n}$ and $\Gamma_{n}$;

- proof of the convergence of $\left(D^{2} \Upsilon_{n}(0)\right)_{n}$, when $\lambda_{s+1}-2 \lambda_{s}<0$;

- proof of the convergence to zero in probability of $\left(D^{2} \Upsilon_{n}(0)-D^{2} \Gamma_{n}(0)\right)_{n}$, when $\lambda_{s+1}-2 \lambda_{s}<0$ and $D \xi(0)\left(\mathbb{R}^{d}\right) \cap \bigoplus_{i=s+1}^{r} E_{i}=\{0\} \mathbb{P}$-almost surely.

2.2.1. Expression of the second order derivative. - More generally, given an integer $n \in \mathbb{N}^{*}$, applications $\varphi_{i}: \Omega \rightarrow \operatorname{Diff}_{0}^{\infty}\left(\mathbb{R}^{N}\right)$ for $i \in\{1, \ldots, n\}$ and a $d$-dimensional subspace $W_{n}$ of $\mathbb{R}^{N}$, one may consider the restriction $\psi_{n}$ of $\varphi_{1} \circ \cdots \circ \varphi_{n}$ to a neighbourhood $\tilde{W}_{n}$ of 0 in $W_{n}$ and its orthogonal normalization

$$
\Psi_{n}:=\psi_{n} \circ\left(p_{n} \circ \psi_{n}\right)^{-1} \circ p_{n},
$$

defined on a neighbourhood $\tilde{U}_{n}$ of 0 in $\mathbb{R}^{N}$, where $p_{n}$ designates the orthogonal projection of $\mathbb{R}^{N}$ onto $\operatorname{Im}\left(D \psi_{n}(0)\right)$.

These maps will be used in two cases:

Case $1: \varphi_{i}=\phi\left(\theta^{-i}\right)$ for $i \in\{1, \ldots, n\}$ and $W_{n}=E^{s}\left(\theta^{-n}\right)$. In this case, $\Psi_{n}=\Upsilon_{n}$.

Case $2: \varphi_{i}=\phi\left(\theta^{-i}\right)$ for $i \in\{1, \ldots, n-1\}, \varphi_{n}=\xi\left(\theta^{-(n-1)}\right)$ and $W_{n}=\mathbb{R}^{d}$. Then $\Psi_{n}=\Gamma_{n-1}$.

To shorthand the notations, set:

- $\varphi_{j, k}= \begin{cases}\varphi_{j} \circ \cdots \circ \varphi_{k} & \text { if } 0 \leq j \leq k \leq n-1, \\ \text { Id } & \text { if } j=k+1\end{cases}$

- $\psi_{n, \|}=p_{n} \circ \psi_{n}$;

- $B^{(k)}=D^{k} \phi(0)$ and $\xi^{(k)}=D^{k} \xi(0)\left(D \xi^{-1}(0)\right)^{\otimes k}$ for every $k \in \mathbb{N} \backslash\{0,1\}$.

For $z \in \tilde{U}_{n}$

$$
\begin{aligned}
& D \Psi_{n}(z)=D \psi_{n}\left(\psi_{n, \|}^{-1} \circ p_{n}(z)\right) D \psi_{n, \|}^{-1}\left(p_{n}(z)\right) p_{n}, \\
& \begin{aligned}
D^{2} \Psi_{n}(z)=D^{2} \psi_{n}\left(\psi_{n, \|}^{-1} \circ\right. & \left.p_{n}(z)\right)\left(D \psi_{n, \|}^{-1}\left(p_{n}(z)\right) p_{n}, D \psi_{n, \|}^{-1}\left(p_{n}(z)\right) p_{n}\right) \\
& +D \psi_{n}\left(\psi_{n, \|}^{-1} \circ p_{n}(z)\right) D^{2} \psi_{n, \|}^{-1}\left(p_{n}(z)\right)\left(p_{n}, p_{n}\right) .
\end{aligned}
\end{aligned}
$$

The formula

$$
D^{2} \psi_{n, \|}^{-1}(z)=-D \psi_{n, \|}^{-1}(z) D^{2} \psi_{n, \|}\left(\psi_{n, \|}^{-1}(z)\right)\left(D \psi_{n, \|}^{-1}(z), D \psi_{n, \|}^{-1}(z)\right)
$$

implies that

(6) $D^{2} \Psi_{n}(z)=\kappa_{n}(z) D^{2} \psi_{n}\left(\psi_{n, \|}^{-1} \circ p_{n}(z)\right)\left(D \psi_{n, \|}^{-1}\left(p_{n}(z)\right) p_{n}, D \psi_{n, \|}^{-1}\left(p_{n}(z)\right) p_{n}\right)$, 
where $\kappa_{n}(z)=I-D \psi_{n}\left(\psi_{n, \|}^{-1} \circ p_{n}(z)\right) D \psi_{n, \|}^{-1}\left(p_{n}(z)\right) p_{n}$. For $z \in \tilde{W}_{n}$, the expression of $D^{2} \psi_{n}(z)$ in terms of the derivatives of the applications $\varphi_{j}$ is

$$
D^{2} \psi_{n}(z)=\sum_{k=1}^{n} D \varphi_{1, k-1}\left(\varphi_{k, n}(z)\right) D^{2} \varphi_{k}\left(\varphi_{k+1, n}(z)\right)\left(D \varphi_{k+1, n}(z), D \varphi_{k+1, n}(z)\right) \text {. }
$$

As $p_{n}$ is the orthogonal projection onto $\operatorname{Im}\left(D \psi_{n}(0)\right)$,

$$
\begin{aligned}
& \kappa_{n}(0)=\mathrm{I}-D \psi_{n}(0) D \psi_{n, \|}^{-1}(0) p_{n}=\mathrm{I}-p_{n}, \\
& D \varphi_{k+1, n}(0) D \psi_{n, \|}^{-1}(0) p_{n}=D \varphi_{1, k}^{-1}(0) p_{n} .
\end{aligned}
$$

Therefore,

$$
D^{2} \Psi_{n}(0)=\kappa_{n} \sum_{k=1}^{n} D \varphi_{1, k-1}(0) D^{2} \varphi_{k}(0)\left(D \varphi_{1, k}^{-1}(0) p_{n}, D \varphi_{1, k}^{-1}(0) p_{n}\right) .
$$

In Case 1, the result is:

$$
D^{2} \Upsilon_{n}(0)=\sum_{k=1}^{n}(\mathrm{I}-\Pi) A_{k-1}\left(\theta^{-(k-1)}\right) B^{(2)}\left(\theta^{-k}\right)\left(A_{-k} \Pi, A_{-k} \Pi\right) .
$$

In Case 2, the result is:

$$
\begin{aligned}
D^{2} \Gamma_{n}(0)=\sum_{k=1}^{n}\left(\mathrm{I}-\Pi_{n}\right) A_{k-1}\left(\theta^{-(k-1)}\right) B^{(2)}\left(\theta^{-k}\right)\left(A_{-k} \Pi_{n}, A_{-k} \Pi_{n}\right) \\
+\left(\mathrm{I}-\Pi_{n}\right) A_{n}\left(\theta^{-n}\right) \xi^{(2)}\left(\theta^{-n}\right)\left(A_{-n} \Pi_{n}, A_{-n} \Pi_{n}\right) .
\end{aligned}
$$

2.2.2. Convergence of $\left(D^{2} \Upsilon_{n}(0)\right)_{n}$. - Let us now assume that $\log ^{+}\left\|B^{(2)}\right\| \in$ $L^{1}(\Omega, \mathbb{P})$. This assumption ensures that the sequence $\left(\left\|B^{(2)}\left(\theta^{-n}\right)\right\|\right)_{n}$ does not grow exponentially fast to infinity:

LEMmA 2.7. - Let $(\Omega, \mathcal{A}, \mathbb{P})$ be a probability space endowed with a transformation $T: \Omega \rightarrow \Omega$ such that $\mathbb{P}$ is $T$-invariant. Consider a nonnegative random variable $Y$ defined on $(\Omega, \mathcal{A}, \mathbb{P})$. If $\log ^{+}(Y) \in L^{1}(\Omega, \mathbb{P})$ then for all $\epsilon>0$, $\sup _{n \geq 0}\left\{Y\left(T^{n}\right) \mathrm{e}^{-n \epsilon}\right\}$ is $\mathbb{P}$-almost surely finite.

For all $n \in \mathbb{N}, D^{2} \Upsilon_{n}(0)$ is the sum of the first $n$ terms of the series:

$$
\sum_{k=1}^{\infty}(\mathrm{I}-\Pi) A_{k-1}\left(\theta^{-(k-1)}\right) B^{(2)}\left(\theta^{-k}\right)\left(A_{-k} \Pi, A_{-k} \Pi\right)
$$

To prove the convergence of $\left(D^{2} \Upsilon_{n}(0)\right)_{n}$, it is sufficient to show that this series is $\mathbb{P}$-almost surely bounded by a convergent geometric series.

Let $\epsilon>0$. The norm of the $k$-th term of the series is bounded above by

$$
\begin{aligned}
& \left\|(\mathrm{I}-\Pi) A_{k-1}\left(\theta^{-(k-1)}\right) B^{(2)}\left(\theta^{-k}\right)\left(A_{-k} \Pi, A_{-k} \Pi\right)\right\| \\
& \quad \leq\left\|(\mathrm{I}-\Pi) A_{k-1}\left(\theta^{-(k-1)}\right)\right\| \cdot\left\|B^{(2)}\left(\theta^{-k}\right)\right\| \cdot\left\|A_{-k} \Pi\right\|^{2} .
\end{aligned}
$$

BULLETIN DE LA SOCIÉTÉ MATHÉMATIQUE DE FRANCE 
By Lemma 2.7, $\sup _{n \in \mathbb{N}} \mathrm{e}^{-n \epsilon}\left\|B^{(2)}\left(\theta^{-n}\right)\right\|$ is $\mathbb{P}$-almost surely finite. By Oseledets' theorem, $\sup _{n \in \mathbb{N}} \mathrm{e}^{-n\left(-\lambda_{s}+\epsilon\right)}\left\|A_{-n} \Pi\right\|$ is $\mathbb{P}$-almost surely finite. Thus, it remains to bound the first term $\left\|(\mathrm{I}-\Pi) A_{k-1}\left(\theta^{-(k-1)}\right)\right\|$ :

LEMMA 2.8. - For every $\epsilon>0, \sup _{n \geq 0} \mathrm{e}^{-n\left(\lambda_{s+1}+\epsilon\right)}\left\|(\mathrm{I}-\Pi) A_{n}\left(\theta^{-n}\right)\right\|$ is $\mathbb{P}$ almost surely finite.

Proof. - For each $i \in\{1, \ldots, r\}$ and $n \in \mathbb{N}$, the projection $\pi_{i}$ onto $E_{i}$ along $\bigoplus_{j \neq i} E_{j}$ satisfies

$$
\pi_{i} \circ A_{n}\left(\theta^{-n}\right)=A_{n}\left(\theta^{-n}\right) \circ \pi_{i}\left(\theta^{-n}\right)
$$

Therefore,

$$
\begin{aligned}
\left\|(\mathrm{I}-\Pi) A_{n}\left(\theta^{-n}\right)\right\| & =\left\|(\mathrm{I}-\Pi) A_{n}\left(\theta^{-n}\right)\left(\mathrm{I}-\pi^{s}\right)\left(\theta^{-n}\right)\right\| \\
& \leq\left\|A_{n}\left(\theta^{-n}\right)_{\mid \oplus_{i=s+1}^{r} E_{i}\left(\theta^{-n}\right)}\right\| \cdot\left\|\left(\mathrm{I}-\pi^{s}\right)\left(\theta^{-n}\right)\right\| .
\end{aligned}
$$

By Lemmas 2.4 and 2.2,

$$
\sup _{n \in \mathbb{N}} \mathrm{e}^{-\frac{1}{2} n \epsilon}\left\|\mathrm{I}-\pi^{s}\left(\theta^{-n}\right)\right\| \quad \text { and } \quad \sup _{n \in \mathbb{N}} \mathrm{e}^{-n\left(\lambda_{s+1}+\frac{1}{2} \epsilon\right)}\left\|A_{n}\left(\theta^{-n}\right)_{\mid \oplus_{i=s+1}^{r} E_{i}\left(\theta^{-n}\right)}\right\|
$$

are $\mathbb{P}$-almost surely finite.

It follows that there exists a $\mathbb{P}$-almost surely finite variable $C_{\epsilon}$, such that for all $k \in \mathbb{N}$ :

$$
\left\|(\mathrm{I}-\Pi) A_{k-1}\left(\theta^{-(k-1)}\right) B^{(2)}\left(\theta^{-k}\right)\left(A_{-k} \Pi, A_{-k} \Pi\right)\right\| \leq C_{\epsilon} \mathrm{e}^{k\left(\lambda_{s+1}-2 \lambda_{s}+\epsilon\right)} .
$$

Therefore, if $\lambda_{s+1}-2 \lambda_{s}<0$, then the series

$$
\sum_{k=1}^{\infty}(\mathrm{I}-\Pi) A_{k-1}\left(\theta^{-(k-1)}\right) B^{(2)}\left(\theta^{-k}\right)\left(A_{-k} \Pi, A_{-k} \Pi\right)
$$

is a $\mathbb{P}$-almost surely convergent series. This ends the proof of the asymptotic behaviour of $\left(D^{2} \Upsilon_{n}(0)\right)_{n}$.

2.2.3. Convergence of $\left(D^{2} \Gamma_{n}(0)-D^{2} \Upsilon_{n}(0)\right)$ toward 0 . - Let $n$ be an integer. The difference between $D^{2} \Gamma_{n}(0)$ and $D^{2} \Upsilon_{n}(0)$ can be decomposed into six

TOME $129-2001-\mathrm{N}^{\mathrm{O}} 3$ 
terms:

$$
\begin{aligned}
& D^{2} \Gamma_{n}(0)-D^{2} \Upsilon_{n}(0) \\
& =\sum_{k=1}^{n}(\mathrm{I}-\Pi) A_{k-1}\left(\theta^{-(k-1)}\right) B^{(2)}\left(\theta^{-k}\right)\left(A_{-k}\left(\Pi_{n}-\Pi\right), A_{-k}\left(\Pi_{n}-\Pi\right)\right) \\
& \quad+2 \sum_{k=1}^{n}(\mathrm{I}-\Pi) A_{k-1}\left(\theta^{-(k-1)}\right) B^{(2)}\left(\theta^{-k}\right)\left(A_{-k}\left(\Pi_{n}-\Pi\right), A_{-k} \Pi\right) \\
& \quad+\sum_{k=1}^{n}\left(\Pi-\Pi_{n}\right) A_{k-1}\left(\theta^{-(k-1)}\right) B^{(2)}\left(\theta^{-k}\right)\left(A_{-k} \Pi, A_{-k} \Pi\right) \\
& \quad+2 \sum_{k=1}^{n}\left(\Pi-\Pi_{n}\right) A_{k-1}\left(\theta^{-(k-1)}\right) B^{(2)}\left(\theta^{-k}\right)\left(A_{-k}\left(\Pi_{n}-\Pi\right), A_{-k} \Pi\right) \\
& \quad+\sum_{k=1}^{n}\left(\Pi-\Pi_{n}\right) A_{k-1}\left(\theta^{-(k-1)}\right) B^{(2)}\left(\theta^{-k}\right)\left(A_{-k}\left(\Pi_{n}-\Pi\right), A_{-k}\left(\Pi_{n}-\Pi\right)\right) \\
& \quad+\left(\mathrm{I}-\Pi_{n}\right) A_{n}\left(\theta^{-n}\right) \xi^{(2)}\left(\theta^{-n}\right)\left(A_{-n} \Pi_{n}, A_{-n} \Pi_{n}\right)
\end{aligned}
$$

It follows from Proposition 2.1, Lemmas 2.8 and 2.7 that if $\lambda_{s+1}-2 \lambda_{s}<0$, then each of those six terms converges toward zero in probability. Indeed, fix $0<\epsilon<\frac{1}{10}\left(3 \lambda_{s}-\lambda_{s+1}\right)$ and let $\left(C_{\epsilon, n}\right)_{n}$ denote a sequence of random variables that converges to zero in probability:

1) The sequences

$$
\begin{aligned}
& \qquad\left(\sum_{k=1}^{n}\left\|(\mathrm{I}-\Pi) A_{k-1}\left(\theta^{-(k-1)}\right)\right\| \cdot\left\|B^{(2)}\left(\theta^{-k}\right)\right\| \cdot\left\|A_{-k}\left(\Pi_{n}-\Pi\right)\right\|^{2}\right) \\
& \text { and } \\
& \left(\sum_{k=1}^{n}\left\|\left(\Pi-\Pi_{n}\right) A_{k-1}\left(\theta^{-(k-1)}\right)\right\| \cdot\left\|B^{(2)}\left(\theta^{-k}\right)\right\| \cdot\left\|A_{-k}\left(\Pi_{n}-\Pi\right)\right\| \cdot\left\|A_{-k} \Pi\right\|\right)
\end{aligned}
$$

are bounded above by $\left(C_{n, \epsilon} \mathrm{e}^{2 n\left(\lambda_{s+1}-\lambda_{s}+\epsilon\right)} \sum_{k=1}^{n} \mathrm{e}^{k\left(-\lambda_{s+1}+\epsilon\right)}\right)$.

2) The sequences

$$
\begin{aligned}
& \left(\sum_{k=1}^{n}\left\|(\mathrm{I}-\Pi) A_{k-1}\left(\theta^{-(k-1)}\right)\right\| \cdot\left\|B^{(2)}\left(\theta^{-k}\right)\right\| \cdot\left\|A_{-k}\left(\Pi_{n}-\Pi\right)\right\| \cdot\left\|A_{-k} \Pi\right\|\right) \\
& \quad \text { and } \\
& \qquad\left(\sum_{k=1}^{n}\left\|\left(\Pi-\Pi_{n}\right) A_{k-1}\left(\theta^{-(k-1)}\right)\right\| \cdot\left\|B^{(2)}\left(\theta^{-k}\right)\right\| A_{-k} \Pi \|^{2}\right)
\end{aligned}
$$

are bounded above by $\left(C_{n, \epsilon} \mathrm{e}^{n\left(\lambda_{s+1}-\lambda_{s}+\epsilon\right)} \sum_{k=1}^{n} \mathrm{e}^{k\left(-\lambda_{s}+4 \epsilon\right)}\right)$. 
3) The sequence

$$
\left(\sum_{k=1}^{n}\left\|\left(\Pi-\Pi_{n}\right) A_{k-1}\left(\theta^{-(k-1)}\right)\right\| \cdot\left\|B^{(2)}\left(\theta^{-k}\right)\right\| A_{-k}\left(\Pi_{n}-\Pi\right) \|^{2}\right)
$$

is bounded above by $\left(C_{n, \epsilon} \mathrm{e}^{3 n\left(\lambda_{s+1}-\lambda_{s}+\epsilon\right)} \sum_{k=1}^{n} \mathrm{e}^{k\left(\lambda_{s}-2 \lambda_{s+1}+4 \epsilon\right)}\right)$.

4) As

$$
\begin{aligned}
& \left\|(\mathrm{I}-\Pi) A_{n}\left(\theta^{-n}\right) \xi^{(2)}\left(\theta^{-n}\right)\left(A_{-n} \Pi, A_{-n} \Pi\right)\right\| \\
& \leq 8\left\|D^{2} \xi\left(\theta^{-n}\right)(0)\right\| \cdot\left\|D \xi\left(\theta^{-n}\right)^{-1}(0)\right\|^{2} \\
& \quad \times \max \left(\left\|(\mathrm{I}-\Pi) A_{n}\left(\theta^{-n}\right)\right\|,\left\|\left(\Pi_{n}-\Pi\right) A_{n}\left(\theta^{-n}\right)\right\|\right) \\
& \quad \times \max \left(\left\|A_{-n} \Pi\right\| \cdot\left\|A_{-n}\left(\Pi_{n}-\Pi\right)\right\|\right)^{2},
\end{aligned}
$$

the sixth term can be bounded above by $C_{\epsilon, n} \exp \left(n\left(\lambda_{s+1}-2 \lambda_{s}+4 \epsilon\right)\right)$.

2.3. Description of the third order approximation. - By differentiating several times the expression of $D^{2} \Psi_{n}$ (Eq.6), one obtains formulas for higher derivatives of $\Psi_{n}$ as linear combinations of partial sums. But these formulas cannot be used directly, since some of those partial sums do not converge when $\lambda_{s+1}-k \lambda_{s}<0$. A study of the third derivative of $\Psi_{n}$ will illustrate the "summation process" used to transform expressions of the derivatives in a combination of partial sums which converge when $\lambda_{s+1}-k \lambda_{s}<0$.

2.3.1. Expression of the third order derivative of $\Psi_{n}$. - Let us return to the second order derivative of $\Psi_{n}$. For $z \in \mathbb{R}^{N}$ sufficiently near 0 ,

$$
D^{2} \Psi_{n}(z)=\kappa_{n}(z) D^{2} \psi_{n}\left(\psi_{n, \|}^{-1} \circ p_{n}(z)\right)\left(D \psi_{n, \|}^{-1}\left(p_{n}(z)\right) p_{n}, D \psi_{n, \|}^{-1}\left(p_{n}(z)\right) p_{n}\right) .
$$

With the following notations

$$
\begin{aligned}
a_{j, k, n}(z) & :=D \varphi_{j, k}\left(\varphi_{k+1, n} \circ \psi_{n, \|}^{-1} \circ p_{n}(z)\right) \quad \text { for } 1 \leq j, k \leq n, \\
b_{k, n}^{(m)}(z) & :=D^{m} \varphi_{k}\left(\varphi_{k+1, n} \circ \psi_{n, \|}^{-1} \circ p_{n}(z)\right) \quad \text { for } 0 \leq k \leq n \text { and } m \in \mathbb{N} \backslash\{0,1\}, \\
e_{n}(z) & :=D \psi_{n, \|}^{-1}\left(p_{n}(z)\right) p_{n} \quad \text { and } \quad \kappa_{n}(z):=\mathrm{I}-a_{1, n}(z) e_{n}(z),
\end{aligned}
$$

the expression of $D^{2} \Psi_{n}(z)$ is

$$
D^{2} \Psi_{n}(z)=\sum_{k=1}^{n} \kappa_{n}(z) a_{1, k-1, n}(z) b_{k, n}^{(2)}(z)\left(a_{k+1, n, n}(z) e_{n}(z), a_{k+1, n, n}(z) e_{n}(z)\right) .
$$

Before computing the derivative of $z \mapsto D^{2} \Psi_{n}(z)(.,$.$) on a neighbourhood of 0$, let us begin with the derivatives of the elementary applications:

- the derivative of $z \mapsto a_{j, k, n}(z)($.$) is$

$$
D a_{j, k}(.)=\sum_{\ell=j}^{k} a_{j, \ell-1, n} b_{\ell, n}^{(2)}\left(a_{\ell, k, n}(.), a_{\ell+1, n, n} e_{n}\right)
$$

TOME $129-2001-\mathrm{N}^{\mathrm{O}} 3$ 
- the derivative of $z \mapsto b_{k, n}^{(m)}(z)($.$) is$

$$
D b_{k}^{(m)}(.)=b_{k, n}^{(m+1)}\left(a_{k+1, n, n} e_{n}, .\right),
$$

- the derivative of $z \mapsto e_{n}(z)$ is

(9)

$$
\begin{aligned}
D e_{n} & =-D \psi_{n, \|}^{-1}\left(p_{n}\right) D^{2} \psi_{n, \|}\left(\psi_{n, \|}^{-1}\left(p_{n}\right) p_{n}, \psi_{n, \|}^{-1}\left(p_{n}\right) p_{n}\right) \\
& =-\sum_{k=1}^{n} e_{n} a_{1, k-1, n} b_{k, n}^{(2)}\left(a_{k+1, n, n} e_{n}, a_{k+1, n, n} e_{n}\right) .
\end{aligned}
$$

As a consequence,

$$
D \kappa_{n}(.)=-\sum_{\ell=1}^{n} \kappa_{n} a_{1, \ell-1, n} b_{\ell, n}^{(2)}\left(a_{\ell+1, n, n} e_{n}, a_{\ell+1, n, n} e_{n}(.)\right) .
$$

Let us now compute $D^{3} \Psi_{n}$ :

$$
\begin{aligned}
D^{3} \Psi_{n}= & \sum_{k=1}^{n} D\left(\kappa_{n}\right)\left(a_{1, k-1, n} b_{k, n}^{(2)}\left(a_{k+1, n, n} e_{n}, a_{k+1, n, n} e_{n}\right)\right) \\
& +\sum_{k=1}^{n} \kappa_{n} D\left(a_{1, k-1, n}\right)\left(b_{k, n}^{(2)}\left(a_{k+1, n, n} e_{n}, a_{k+1, n, n} e_{n}\right)\right) \\
& +\sum_{k=1}^{n} \kappa_{n} a_{1, k-1, n} D\left(b_{k, n}^{(2)}\right)\left(a_{k+1, n, n} e_{n}, a_{k+1, n, n} e_{n}\right) \\
& +2 \sum_{k=1}^{n} \kappa_{n} a_{1, k-1, n} b_{k, n}^{(2)}\left(D\left(a_{k+1, n, n} e_{n}\right), a_{k+1, n, n} e_{n}\right) .
\end{aligned}
$$

Therefore,

$$
D^{3} \Psi_{n}=\sum_{k=1}^{n} I_{k, n}^{1}+3 \sum_{k=1}^{n} \sum_{\ell=1}^{k-1} I_{\ell, k, n}^{2}-3 \sum_{k=1}^{n} \sum_{\ell=1}^{n} I_{\ell, k, n}^{3}
$$

where

$$
\begin{aligned}
I_{k, n}^{1} & :=\kappa_{n} a_{1, k-1, n} b_{k, n}^{(3)}\left(a_{k+1, n, n} e_{n}\right)^{\otimes 3}, \\
I_{\ell, k, n}^{2} & :=\kappa_{n} a_{1, \ell-1, n} b_{\ell, n}^{(2)}\left(a_{\ell+1, k-1, n} b_{k, n}^{(2)}\left(a_{k+1, n, n} e_{n}\right)^{\otimes 2}, a_{\ell+1, n, n} e_{n}\right), \\
I_{\ell, k, n}^{3} & :=\kappa_{n} a_{1, \ell-1, n} b_{\ell, n}^{(2)}\left(a_{\ell+1, n, n} e_{n} a_{1, k-1, n} b_{k, n}^{(2)}\left(a_{k+1, n, n} e_{n}\right)^{\otimes 2}, a_{\ell+1, n, n} e_{n}\right) .
\end{aligned}
$$

The convergence at 0 of the last two terms cannot be established separately. Let us slightly modify expression (11) by introducing a new map $\tilde{a}_{j, k, n}$, which is equal to $a_{j, k, n}-a_{j, n, n} e_{n} a_{1, k, n}$ for $1<j \leq k \leq n$. At 0 ,

$$
\begin{aligned}
\tilde{a}_{j, k, n}(0) & =D \varphi_{j, k}(0)-D \varphi_{j, n}(0) D \psi_{n, \|}^{-1}(0) p_{n} D \varphi_{1, k}(0) \\
& =D \varphi_{1, j-1}^{-1}(0)\left(\mathrm{I}-p_{n}\right) D \varphi_{1, k}(0) .
\end{aligned}
$$

BULLETIN DE LA SOCIÉTÉ MATHÉMATIQUE DE FRANCE 
Using the applications $\tilde{a}_{j, k, n}$ gives

$$
D^{3} \Psi_{n}=\sum_{k=1}^{n} I_{k, n}^{1}+3 \sum_{k=1}^{n} \sum_{\ell=1}^{k-1} I_{\ell, k, n}^{2^{\prime}}-3 \sum_{k=1}^{n} \sum_{\ell=k}^{n} I_{\ell, k, n}^{3}
$$

with

$$
I_{\ell, k, n}^{2^{\prime}}:=\kappa_{n} a_{1, \ell-1, n} b_{\ell, n}^{(2)}\left(\tilde{a}_{\ell+1, k-1, n} b_{k, n}^{(2)}\left(a_{k+1, n, n} e_{n}\right)^{\otimes 2}, a_{\ell+1, n, n} e_{n}\right) .
$$

The convergence of $\left(D^{3} \Psi_{n}(0)\right)_{n}$ will be the result of the convergence of the three sums in (12).

2.3.2. Convergence of $\left(D^{3} \Upsilon_{n}(0)\right)_{n}$. - Let us now assume that $\log ^{+}\left\|B^{(j)}\right\| \in$ $L^{1}(\Omega, \mathbb{P})$ for $j \in\{2,3\}$. As for the second order approximation, the convergence of the sequences $\left(D^{3} \Upsilon_{n}(0)\right)_{n}$ and $\left(D^{3} \Gamma_{n}(0)-D^{3} \Upsilon_{n}(0)\right)_{n}$ are successively studied.

The expression of $D^{3} \Upsilon_{n}(0)$ is

$$
D^{3} \Upsilon_{n}(0)=\sum_{k=1}^{n} J_{k}^{1}+3 \sum_{k=1}^{n} \sum_{\ell=1}^{k-1} J_{\ell, k}^{2^{\prime}}-3 \sum_{k=1}^{n} \sum_{\ell=k}^{n} J_{\ell, k}^{3}
$$

where

$$
\begin{aligned}
J_{k}^{1} & :=(\mathrm{I}-\Pi) A_{k-1}\left(\theta^{-(k-1)}\right) B^{(3)}\left(\theta^{-k}\right)\left(A_{-k} \Pi\right)^{\otimes 3}, \\
J_{\ell, k}^{2^{\prime}}: & =(\mathrm{I}-\Pi) A_{\ell-1}\left(\theta^{-(\ell-1)}\right) \\
& B^{(2)}\left(\theta^{-\ell}\right)\left(\tilde{a}_{\ell+1, k-1, n}(0)\right) B^{(2)}\left(\theta^{-k}\right)\left(\left(A_{-k} \Pi\right)^{\otimes 2}, A_{-\ell} \Pi\right), \\
J_{\ell, k}^{3}: & (\mathrm{I}-\Pi) A_{\ell-1}\left(\theta^{-(\ell-1)}\right) B^{(2)}\left(\theta^{-\ell}\right)\left(A_{-\ell} \Pi A_{k-1}\left(\theta^{-(k-1)}\right)\right. \\
& B^{(2)}\left(\theta^{-k}\right)\left(\left(A_{-k} \Pi\right)^{\otimes 2}, A_{-\ell} \Pi\right) .
\end{aligned}
$$

To shorthand the notations, let $\tilde{A}_{\ell, k}$ denote $\tilde{a}_{\ell+1, k, n}(0)$ in Case 1 ; namely,

$$
\tilde{A}_{\ell, k}=A_{-\ell}(\mathrm{I}-\Pi) A_{k}\left(\theta^{-k}\right) .
$$

To bound the general terms of the three sums in (13), it remains to establish upper bounds for $\left\|\tilde{A}_{\ell, k}\right\|$ if $\ell<k$ and for $\left\|A_{-\ell} \Pi A_{k}\left(\theta^{-k}\right)\right\|$ if $\ell \geq k$. A bound for $\left\|\tilde{A}_{\ell, k}\right\|$ can be deduced from the following corollary of the subadditive ergodic theorem established by D. Ruelle [15, p. 288]:

Theorem 2.9 (D. Ruelle, 1982). - Let $(\Omega, \mathcal{A}, \mathbb{P})$ be a probability space, let $T: \Omega \rightarrow \Omega$ be a measurable map preserving $\mathbb{P}$ and let $\left(X_{n}\right)_{n \geq 1}$ be a sequence of measurable functions from $\Omega$ into $\mathbb{R} \cup\{-\infty\}$, such that $X_{1}^{+} \in L^{1}(\Omega, \mathbb{P})$ and $\left(X_{n}\right)_{n \geq 1}$ is subadditive. Let $X: \Omega \rightarrow \mathbb{R} \cup\{-\infty\}$ denote a T-invariant application such that $X^{+} \in L^{1}(\Omega, \mathbb{P})$ and $\lim _{n \rightarrow \infty} \frac{1}{n} X_{n}=X \mathbb{P}$-almost everywhere (the existence of such an application follows from the subadditive ergodic theorem). If $Y \geq X$ is a T-invariant measurable finite-valued application, then for every 
$\epsilon>0$, there exists a measurable finite-valued function $K_{\epsilon}: \Omega \rightarrow \mathbb{R}$, such that for almost every $\omega \in \Omega$ and $0<m \leq n$,

$$
X_{n-m}\left(T^{m}(\omega)\right) \leq(n-m) Y(\omega)+n \epsilon+K_{\epsilon}(\omega) .
$$

LEMMA 2.10. - For every $\epsilon>0$, there exists a $\mathbb{P}$-almost surely random variable $C_{\epsilon}$, such that for all $0<i<j$

$$
\left\|\tilde{A}_{i, j}\right\| \leq C_{\epsilon} \mathrm{e}^{(j-i) \lambda_{s+1}+(i+j) \epsilon}
$$

Proof. - Let $\epsilon>0$. As $A_{j}\left(\theta^{-j}\right) E^{s}\left(\theta^{-j}\right)=E^{s}$ for every $j \in \mathbb{N}$, one obtains

$$
\begin{aligned}
\tilde{A}_{i, j} & =A_{-i}(\mathrm{I}-\Pi) A_{j}\left(\theta^{-j}\right)\left(\mathrm{I}-\pi^{s}\left(\theta^{-j}\right)\right) \\
= & A_{j-i}\left(\theta^{-j}\right)\left(\mathrm{I}-\pi^{s}\left(\theta^{-j}\right)\right)-A_{-i} \Pi A_{j}\left(\theta^{-j}\right)\left(\mathrm{I}-\pi^{s}\left(\theta^{-j}\right)\right) .
\end{aligned}
$$

Consider the first term. Theorem 2.9 (applied to the subadditive sequence $\left.\left(\log \left\|A_{n}\left(\theta^{-n}\right)_{\mid \oplus_{i=s+1}^{r} E_{i}\left(\theta^{-n}\right)}\right\|\right)_{n}\right)$ and Lemma 2.4 insure that there exists a $\mathbb{P}$ almost surely random variable $C_{\epsilon}$, such that for all $0<i<j$,

$$
\begin{aligned}
\left\|A_{j-i}\left(\theta^{-j}\right)\left(\mathrm{I}-\pi^{s}\left(\theta^{-j}\right)\right)\right\| & \leq\left\|A_{j-i}\left(\theta^{-j}\right)_{\mid \oplus_{k=s+1}^{r} E_{k}\left(\theta^{-j}\right)}\right\| \cdot\left\|\left(\mathrm{I}-\pi^{s}\left(\theta^{-j}\right)\right)\right\| \\
& \leq C_{\epsilon} \mathrm{e}^{(j-i) \lambda_{s+1}+j \epsilon} .
\end{aligned}
$$

The second term can be bounded as follows:

$$
\begin{aligned}
& \left\|A_{-i} \Pi A_{j}\left(\theta^{-j}\right)\left(\mathrm{I}-\pi^{s}\left(\theta^{-j}\right)\right)\right\| \\
& \quad \leq\left\|A_{-i \mid E^{s}}\right\| \cdot\left\|A_{j}\left(\theta^{-j}\right)_{\mid \oplus_{k=s+1}^{r} E_{k}\left(\theta^{-j}\right)}\right\| \cdot\left\|\left(\mathrm{I}-\pi^{s}\left(\theta^{-j}\right)\right)\right\| .
\end{aligned}
$$

Thus there exists a $\mathbb{P}$-almost surely random variable $C_{\epsilon}$ such that for every $0<i<j,\left\|A_{-i} \Pi A_{j}\left(\theta^{-j}\right)\left(\mathrm{I}-\pi^{s}\left(\theta^{-j}\right)\right)\right\| \leq C_{\epsilon} \mathrm{e}^{j\left(\lambda_{s+1}+\epsilon\right)+i\left(-\lambda_{s}+\epsilon\right)}$.

The following lemma presents an upper bound of $\left\|A_{-\ell} \Pi A_{k}\left(\theta^{-k}\right)\right\|$ for every $0 \leq k \leq \ell$ :

LEMMA 2.11. - For every $\epsilon>0$, there exists a $\mathbb{P}$-almost surely finite random variable $C_{\epsilon}$ such that for all $0 \leq k \leq \ell$,

$$
\left\|A_{-\ell} \Pi A_{k}\left(\theta^{-k}\right)\right\| \leq C_{\epsilon} \mathrm{e}^{(k-\ell) \lambda_{s}+(k+\ell) \epsilon} .
$$

Proof. - Let $\epsilon>0$ and $k, \ell \in \mathbb{N}$. A decomposition of $A_{-\ell} \Pi A_{k}\left(\theta^{-k}\right)$ with respect to Oseledets' splitting gives

$$
A_{-\ell} \Pi A_{k}\left(\theta^{-k}\right)=\sum_{i=1}^{s} A_{-\ell} \pi_{i} A_{k}\left(\theta^{-k}\right)+A_{-\ell} \Pi\left(\mathrm{I}-\pi^{s}\right) A_{k}\left(\theta^{-k}\right) .
$$

For every $i \in\{1, \ldots, s\}$,

$$
\begin{aligned}
\left\|A_{-\ell} \pi_{i} A_{k}\left(\theta^{-k}\right)\right\| & \leq\left\|A_{-\ell \mid E_{i}}\right\| \cdot\left\|\pi_{i}\right\| \cdot\left\|A_{k}\left(\theta^{-k}\right){ }_{\mid E_{i}\left(\theta^{-k}\right)}\right\| \cdot\left\|\pi_{i}\left(\theta^{-k}\right)\right\| \\
& \leq C_{\epsilon} \mathrm{e}^{\ell\left(-\lambda_{i}+\epsilon\right)+k\left(\lambda_{i}+\epsilon\right)} .
\end{aligned}
$$

BULLETIN DE LA SOCiÉTÉ MATHÉMATIQUe DE FRANCE 
If $0 \leq k \leq \ell$, then for $i \in\{1, \ldots, s\},(k-\ell) \lambda_{i} \leq(k-\ell) \lambda_{s}$, whence

$$
\left\|A_{-\ell} \pi_{i} A_{k}\left(\theta^{-k}\right)\right\| \leq C_{\epsilon} \mathrm{e}^{(k-\ell) \lambda_{s}+(l+k) \epsilon} .
$$

Moreover, for all $\ell, k \in \mathbb{N}$,

$$
\begin{aligned}
\left\|A_{-\ell} \Pi\left(\mathrm{I}-\pi^{s}\right) A_{k}\left(\theta^{-k}\right)\right\| & \leq\left\|A_{-\ell \mid E^{s}}\right\| \cdot\left\|A_{k}\left(\theta^{-k}\right)_{\mid \oplus_{i=s+1}^{r} E_{i}\left(\theta^{-k}\right)}\right\| \cdot\left\|\mathrm{I}-\pi^{s}\left(\theta^{-k}\right)\right\| \\
& \leq C_{\epsilon} \mathrm{e}^{\ell\left(-\lambda_{s}+\epsilon\right)+k\left(\lambda_{s+1}+\epsilon\right)} .
\end{aligned}
$$

Therefore, for all $0 \leq k \leq \ell,\left\|A_{-\ell} \Pi A_{k}\left(\theta^{-k}\right)\right\| \leq C_{\epsilon} \mathrm{e}^{(k-\ell) \lambda_{s}+(k+\ell) \epsilon}$.

Inequalities given by Oseledets' theorem and Lemmas 2.7, 2.8, 2.10 and 2.11 lead to the following bounds:

$$
\begin{aligned}
& \left\|J_{i}^{1}\right\| \leq C_{\epsilon} \mathrm{e}^{i\left(\lambda_{s+1}-3 \lambda_{s}+4 \epsilon\right)} \quad \text { for all } i \in \mathbb{N}^{*}, \\
& \left\|J_{i, j}^{2^{\prime}}\right\| \leq C_{\epsilon} \mathrm{e}^{j\left(\lambda_{s+1}-2 \lambda_{s}+3 \epsilon\right)} \mathrm{e}^{i\left(-\lambda_{s}+4 \epsilon\right)} \text { for all } 1 \leq i<j, \\
& \left\|J_{i, j}^{3}\right\| \leq C_{\epsilon} \mathrm{e}^{j\left(-\lambda_{s}+4 \epsilon\right)} \mathrm{e}^{i\left(\lambda_{s+1}-2 \lambda_{s}+4 \epsilon\right)} \text { for all } 1 \leq j \leq i \text {. }
\end{aligned}
$$

It turns out that the sequences

$$
\left(\sum_{i=1}^{n}\left\|J_{i}^{1}\right\|\right)_{n}, \quad\left(\sum_{1 \leq i<j \leq n}\left\|J_{i, j}^{2^{\prime}}\right\|\right)_{n}, \quad\left(\sum_{1 \leq j \leq i \leq n}\left\|J_{i, j}^{3}\right\|\right)_{n}
$$

converge $\mathbb{P}$-almost surely, if $\lambda_{s+1}-3 \lambda_{s}<0$ :

Proposition 2.12. - If $\log ^{+}\left\|A^{ \pm 1}\right\|, \log ^{+}\left\|B^{(2)}\right\|$ and $\log ^{+}\left\|B^{(3)}\right\|$ lie in $L^{1}(\Omega, \mathbb{P})$ and if $\lambda_{s+1}-3 \lambda_{s}<0$, then $\left(D^{3} \Upsilon_{n}(0)\right)_{n \geq 0}$ converges $\mathbb{P}$-almost surely to

$$
S^{(3)}:=\sum_{i=1}^{+\infty} J_{i}^{1}+3 \sum_{1 \leq i<j} J_{i, j}^{2^{\prime}}-3 \sum_{1 \leq j \leq i} J_{i, j}^{3} .
$$

2.3.3. Convergence of $\left(D^{3} \Gamma_{n}(0)-D^{3} \Upsilon_{n}(0)\right)_{n}$ to zero. - The expression of $D^{3} \Gamma_{n}(0)$ is

$$
\begin{aligned}
D^{3} \Gamma_{n}(0)=\sum_{k=1}^{n} I_{k, n+1}^{1}+3 \sum_{k=1}^{n} \sum_{\ell=1}^{k-1} I_{\ell, k, n+1}^{2^{\prime}}-3 \sum_{k=1}^{n} \sum_{\ell=k}^{n} I_{\ell, k, n+1}^{3} \\
\quad+I_{n+1, n+1}^{1}+3 \sum_{\ell=1}^{n} I_{\ell, n+1, n+1}^{2^{\prime}}-3 \sum_{k=1}^{n} I_{n+1, k, n+1}^{3}-3 I_{n+1, n+1, n+1}^{3}
\end{aligned}
$$

TOME $129-2001-\mathrm{N}^{\mathrm{O}} 3$ 
where, for $1 \leq \ell, k \leq n$

$$
\begin{aligned}
& I_{k, n+1}^{1}=\left(\mathrm{I}-\Pi_{n}\right) A_{k-1}\left(\theta^{-(k-1)}\right) B^{(3)}\left(\theta^{-k}\right)\left(A_{-k} \Pi_{n}\right)^{\otimes 3} \\
& I_{\ell, k, n+1}^{2^{\prime}}=\left(\mathrm{I}-\Pi_{n}\right) A_{\ell-1}\left(\theta^{-(\ell-1)}\right) B^{(2)}\left(\theta^{-\ell}\right)\left(\tilde{a}_{\ell, k-1, n+1}(0)\right. \\
& B^{(2)}\left(\theta^{-k}\right)\left(\left(A_{-k} \Pi_{n}\right)^{\otimes 2}, A_{-\ell} \Pi_{n}\right), \\
& I_{\ell, k, n+1}^{3}=\left(\mathrm{I}-\Pi_{n}\right) A_{\ell-1}\left(\theta^{-(\ell-1)}\right) B^{(2)}\left(\theta^{-\ell}\right)\left(A_{-\ell} \Pi_{n} A_{k-1}\left(\theta^{-(k-1)}\right)\right. \\
& B^{(2)}\left(\theta^{-k}\right)\left(\left(A_{-k} \Pi_{n}\right)^{\otimes 2}, A_{-\ell} \Pi_{n}\right) \\
& I_{n+1, n+1}^{1}=\left(\mathrm{I}-\Pi_{n}\right) A_{n}\left(\theta^{-n}\right) \xi^{(3)}\left(\theta^{-n}\right)\left(A_{-n} \Pi_{n}\right)^{\otimes 3} \\
& I_{\ell, n+1, n+1}^{2^{\prime}}=\left(\mathrm{I}-\Pi_{n}\right) A_{\ell-1}\left(\theta^{-(\ell-1)}\right) B^{(2)}\left(\theta^{-\ell}\right)\left(\tilde{a}_{\ell, n+1, n+1}(0)\right. \\
& \xi^{(2)}\left(\theta^{-n}\right)\left(\left(A_{-n} \Pi_{n}\right)^{\otimes 2}, A_{-\ell} \Pi_{n}\right) \\
& I_{n+1, k, n+1}^{3}=\left(\mathrm{I}-\Pi_{n}\right) A_{n}\left(\theta^{-n}\right) \xi^{(2)}\left(\theta^{-n}\right) A_{-n} \Pi_{n} A_{k-1}\left(\theta^{-(k-1)}\right) \\
& B^{(2)}\left(\theta^{-k}\right)\left(\left(A_{-k} \Pi_{n}\right)^{\otimes 2}, A_{-n} \Pi_{n}\right) \\
& I_{n+1, n+1, n+1}^{3}=\left(\mathrm{I}-\Pi_{n}\right) A_{n}\left(\theta^{-n}\right) \xi^{(2)}\left(\theta^{-n}\right)\left(A_{-n} \Pi_{n} A_{n}\left(\theta^{-n}\right)\right. \\
& \xi^{(2)}\left(\theta^{-n}\right)\left(\left(A_{-n} \Pi_{n}\right)^{\otimes 2}, A_{-n} \Pi_{n}\right) .
\end{aligned}
$$

As for the convergence of the second derivative of $\Gamma_{n}$ at 0 , one may prove that if $\lambda_{s+1}-3 \lambda_{s}<0$ then

- the sequences

$$
\left(\sum_{k=1}^{n} I_{k, n+1}^{1}\right)_{n}, \quad\left(\sum_{1 \leq \ell<k \leq n} I_{\ell, k, n+1}^{2^{\prime}}\right)_{n}, \quad\left(\sum_{1 \leq k \leq \ell \leq n} I_{\ell, k, n+1}^{3}\right)_{n}
$$

converge respectively in probability to the sums

$$
\sum_{k=1}^{\infty} J_{k}^{1}, \quad \sum_{1 \leq l<k} J_{\ell, k}^{2^{\prime}}, \quad \sum_{1 \leq k \leq l} J_{\ell, k}^{3} ;
$$

- each term which derives from the nonlinear part of the initial parametrization $\xi$, that is

$$
I_{n+1, n+1}^{1}, \quad \sum_{\ell=1}^{n} I_{\ell, n+1, n+1}^{2^{\prime}}, \quad \sum_{k=1}^{n} I_{n+1, k, n+1}^{3} \quad \text { or } \quad I_{n+1, n+1, n+1}^{3}
$$

converges to 0 in probability.

\section{Proof of Theorem 1.7 for approximations of all orders}

As for the second and third approximations, the description of higher approximations proceeds in three steps:

BULletin DE LA SOCiÉtÉ MATHÉMATIQUE DE FRANCE 
- firstly, computation of an expression of the derivatives of $\Psi_{n}$ using the summation process introduced for the description of the third derivative;

- secondly, proof of the $\mathbb{P}$-almost surely convergence of $\left(D^{k} \Upsilon_{n}(0)\right)_{n}$ when $\lambda_{s+1}-k \lambda_{s}<0$. It will follow from the convergence of all sums in the expression of the derivatives of $\Psi_{n}$ at 0 ;

- finally, proof of the convergence of $\left(D^{k} \Upsilon_{n}(0)-D^{k} \Gamma_{n}(0)\right)_{n}$ to zero in probability if $\lambda_{s+1}-k \lambda_{s}<0$ and if $D \xi(0)\left(\mathbb{R}^{d}\right) \cap \bigoplus_{i=s+1}^{r} E_{i}=\{0\} \mathbb{P}$-almost surely.

The upper bounds, required in these last two steps, have already been established in Section 2: Lemmas 2.2, 2.7, 2.8, 2.10, 2.11 and Proposition 2.1.

3.1. Expression of the derivatives of $\Psi_{\boldsymbol{n}}$. - The derivatives of $\Psi_{n}$ will be expressed with the aid of the applications $a_{i, j, n}, \tilde{a}_{i, j, n}, e_{n}, \kappa_{n}$ and $b_{k, n}^{(m)}$, where the integers $i, j, k, m$ satisfy $1 \leq j \leq i \leq n, 1 \leq k \leq n$ and $m \geq 2$. The structure of the terms of the derivatives of $\Psi_{n}$ built with these four applications will be described with the help of diagrams. Let us begin with some general definitions about diagrams.

3.1.1. Diagrams. - A diagram consists of a finite set $X$ whose elements are called "nodes" and a finite number of subsets $E_{1}, \ldots, E_{r}$ of $X \times X$ whose elements are called "links". Such a diagram will be denoted by $D=\left(X, E_{1}, \ldots, E_{r}\right)$. Let $E$ denote the set of all links of $D$. A node $y \in X$ is called a "descendant" of a node $x \in X$, if there exists a path in $E$ going from $x$ to $y$, i.e. a finite number of nodes $x=x_{1}, \ldots, x_{r}=y$ such that for all $i \in\{1, \ldots, r-1\},\left(x_{i}, x_{i+1}\right) \in E$. If $(x, y) \in E$, then $y$ is called a son of $x$ and $x$ is a "predecessor" of $y$. A node that has no predecessor is called a "root". A node that has no son is called a "leaf" or a "terminal node", otherwise it is called an "interior node". Finally, a subset $S$ of $X$ is said "closed in D" if every descendant (for the diagram $D$ ) of an element of $S$ belongs to $S$.

Two diagrams $D^{1}=\left(X^{1}, E_{1}^{1}, \ldots, E_{p}^{1}\right)$ and $D^{2}=\left(X^{2}, E_{1}^{2}, \ldots, E_{p}^{2}\right)$ are said equivalent if there exists a one-to-one correspondence $F$ between $X^{1}$ and $X^{2}$, such that for all $i \in\{1, \ldots, p\},(u, v) \in E_{i}^{1}$ if and only if $(F(u), F(v)) \in E_{i}^{2}$. Equivalent diagrams will not always be distinguished: a diagram $D$ could just as well represent an equivalence class of diagrams as an element of this class.

3.1.1.1. Trees. - The diagram $D$ is called a "tree" if it has a unique root and if for every $y \in X \backslash\{x\}$, there is exactly one path in $E$ connecting $x$ to $y$. A node $x$, with the exception of the root, has a unique predecessor denoted by $p(x)$.

3.1.1.2. Subclass $\mathcal{D}$ of diagrams. - The description of the derivatives of $\Psi_{n}$ will only use a particular class of diagrams having four types of links, denoted by $\mathcal{D}: D$ is a diagram of the subclass $\mathcal{D}$ if $D=\left(X, E_{1}, E_{2}, I_{1}, I_{2}\right)$ where $E_{1}$,

TOME $129-2001-\mathrm{N}^{\mathrm{O}} 3$ 
$E_{2}, I_{1}$ and $I_{2}$ are subsets of $(X \times X) \backslash\{(x, x), x \in X\}$ satisfying the following properties:

- $T=\left(X, E_{1} \cup E_{2}\right)$ is a tree whose interior nodes have at least two sons and such that $(p(x), x) \in E_{2}$ if $x$ is a leaf;

- $I_{1}$ and $I_{2}$ are transitive and asymmetric sets, $I_{2} \subseteq I_{1}$ and if $(x, y),(y, z)$ are elements of $I_{1}$ such that one of them belongs to $I_{2}$, then $(x, z) \in I_{2}$.

The root of $T$ will be denoted by $\rho(D)$, the set of interior nodes for the $\operatorname{diagram}\left(X, E_{1} \cup E_{2}\right)$ by $\mathfrak{i}(D)$.

The set

$$
\mathfrak{i}(D)^{*}:=\mathfrak{i}(D) \backslash\{\rho(D)\}
$$

can be split into two sets, $\mathfrak{i}_{1}(D)$ and $\mathfrak{i}_{2}(D)$ depending on whether the link between a node $x$ and its predecessor is in $E_{1}$ or $E_{2}$ : for $j \in\{1,2\}$, set

$$
\mathfrak{i}_{j}(D)=\left\{x \in \mathfrak{i}(D)^{*} \text { such that }(p(x), x) \in E_{j}\right\} \text {. }
$$

For $u \in \mathfrak{i}(D), D_{u}$ will be the subdiagram which is the restriction of the diagram $D$ to the node $u$ and its descendants. The number of sons of $u$ will be denoted by $m_{u}$, the number of sons of $u$ that are interior nodes by $r_{u}$, the number of sons of $u$ that belong to $\mathfrak{i}_{1}(D)$ by $r_{u}^{(1)}$, the number of sons of $u$ that belong to $\mathfrak{i}_{2}(D)$ by $r_{u}^{(2)}$ and the number of sons of $u$ that are leaves by $\ell_{u}$.

To shorthand the notations, the following notations will be used for any subset $Y$ of $X$ :

$$
\begin{aligned}
\mathfrak{i}(Y) & :=Y \cap \mathfrak{i}(D), & m(Y) & :=\sum_{v \in \mathfrak{i}(Y)} m_{v}, \\
r(Y) & :=\sum_{v \in \mathfrak{i}(Y)} r_{v}, & \ell(Y) & :=\sum_{v \in \mathfrak{i}(Y)} \ell_{v} . \\
r^{(x)}(Y) & :=\sum_{v \in \mathfrak{i}(Y)} r_{v}^{(x)} & \text { for } x \in\{1,2\} . &
\end{aligned}
$$

When $Y=X$, the following notations will be used:

$$
|D|:=|X|, \quad m(D):=m(X), \quad \ell(D):=\ell(X), \quad r(D):=r(X) .
$$

It may be seen that

$$
|D|=1+m(D), \quad|\mathfrak{i}(D)|=r(D)+1 \quad \text { and } \quad|\mathfrak{i}(D)| \leq \ell(D)-1 .
$$

3.1.1.3. Height of a node. - A diagram $D=\left(X, E_{1}, E_{2}, I_{1}, I_{2}\right) \in \mathcal{D}$ will be endowed with a family $h=\left(h_{u}\right)_{u \in \mathfrak{i}(D)}$ of integers indexed by the interior nodes of $D$ and satisfying $h_{u} \leq h_{v}$ if $(u, v) \in I_{1}$ and $h_{u}<h_{v}$ if $(u, v) \in I_{2}$. The integer $h_{u}$ of $h$ will be termed the height of the node $u$ in the labelled diagram $(D ; h)$. The set of such families of integers will be denoted by $H(D)$. Let

$$
H_{n}(D):=H(D) \cap\{1, \ldots, n\}^{|\mathfrak{i}(D)|}
$$

for $n \in \mathbb{N}^{*}$. A diagram endowed with a family of integers belonging to $H_{n}(D)$ is said a "diagram of height at most n".

BULletin DE LA SOCiÉtÉ MATHÉMATIQUE DE FRANCE 
3.1.1.4. Graphic representation of a diagram of $\mathcal{D}$. - The nodes of $D$ will be drawn as points such that if $(x, y) \in E_{1} \cup E_{2}$, the ordinate of the point $x$ is smaller than the ordinate of $y$. A pair $(x, y)$ will be represented by a simple line between $x$ and $y$ if $(x, y) \in E_{1}$ and by a double line if $(x, y) \in E_{2}$. An element $(x, y)$ of $I_{1}$ will be drawn as an arrow from $x$ to $y$, with two heads if $(x, y) \in I_{2}$ and with one head if $(x, y) \in I_{1} \backslash I_{2}$. Such a representation is given Figure 1 .

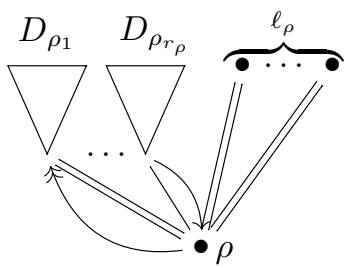

Figure 1. A diagram $D$ with root $\rho, \rho_{1}, \ldots, \rho_{r_{\rho}}$ designating the non-terminal sons of $\rho$

3.1.1.5. Application associated with a diagram. - Let $D=\left(X, E_{1}, E_{2}, I_{1}, I_{2}\right)$ be a diagram in $\mathcal{D}$ endowed with a family $h=\left(h_{u}\right)_{u \in \mathfrak{i}(D)}$ of heights smaller or equal to $n$. With the help of the labelled diagram $(D ; h)$ one defines an application $f_{n}(D ; h)$ by

$$
\begin{aligned}
f_{n}(D ; h)=\kappa_{n} a_{1, h_{\rho}-1, n} b_{h_{\rho}, n}^{\left(m_{\rho}\right)}\left(f_{n, h_{\rho}}\left(D_{\rho_{1}} ; h_{\mid D_{\rho_{1}}}\right), \ldots,\right. \\
\left.f_{n, h_{\rho}}\left(D_{\rho_{r}} ; h_{\mid D_{\rho_{r}}}\right)\left(a_{h_{\rho}+1, n, n} e_{n}\right)^{\otimes \ell_{\rho}}\right)
\end{aligned}
$$

where

- $\rho_{1}, \ldots, \rho_{r}$ designate the non-terminal sons of the root $\rho$ of $D$;

- the applications $f_{n, z}\left(D_{u}, h_{\mid D_{u}}\right)$ for $z \in\{1, \ldots, n\}$ are defined by induction by

$$
\begin{aligned}
& f_{n, z}\left(D_{u} ; h_{\mid D_{u}}\right)=\tilde{a}_{z+1, h_{u}-1, n} b_{h_{u}, n}^{\left(m_{u}\right)}\left(f_{n, h_{u}}\left(D_{u_{1}} ; h_{\mid D_{u_{1}}}\right), \ldots,\right. \\
& \left.f_{n, h_{u}}\left(D_{u_{r_{u}}} ; h_{\mid D_{u_{r_{u}}}}\right),\left(a_{h_{u}+1, n, n} e_{n}\right)^{\otimes \ell_{u}}\right) \\
& \text { if } u \in \mathfrak{i}_{2}(D) \text {, } \\
& f_{n, z}\left(D_{u} ; h_{\mid D_{u}}\right)=a_{z+1, n, n} e_{n} a_{1, h_{u}-1, n} b_{h_{u}, n}^{\left(m_{u}\right)}\left(f_{n, h_{u}}\left(D_{u_{1}} ; h_{\mid D_{u_{1}}}\right), \ldots\right. \text {, } \\
& \left.f_{n, h_{u}}\left(D_{u_{r_{u}}} ; h_{\mid D_{u_{r_{u}}}}\right),\left(a_{h_{u}+1, n, n} e_{n}\right)^{\otimes \ell_{u}}\right) \\
& \text { if } u \in \mathfrak{i}_{1}(D) \text {, }
\end{aligned}
$$

where $u_{1}, \ldots, u_{r_{u}}$ designate the non-terminal sons of $u$.

Finally, for $n \in \mathbb{N}$ and $D \in \mathcal{D}$, let

$$
F_{n}(D):=\sum_{h \in H_{n}(D)} f_{n}(D ; h) .
$$

TOME $129-2001-\mathrm{N}^{\mathrm{O}} 3$ 
Table 1 shows how to associate elementary applications of $f_{n}(D ; h)$ with the different parts of the diagram $D$.

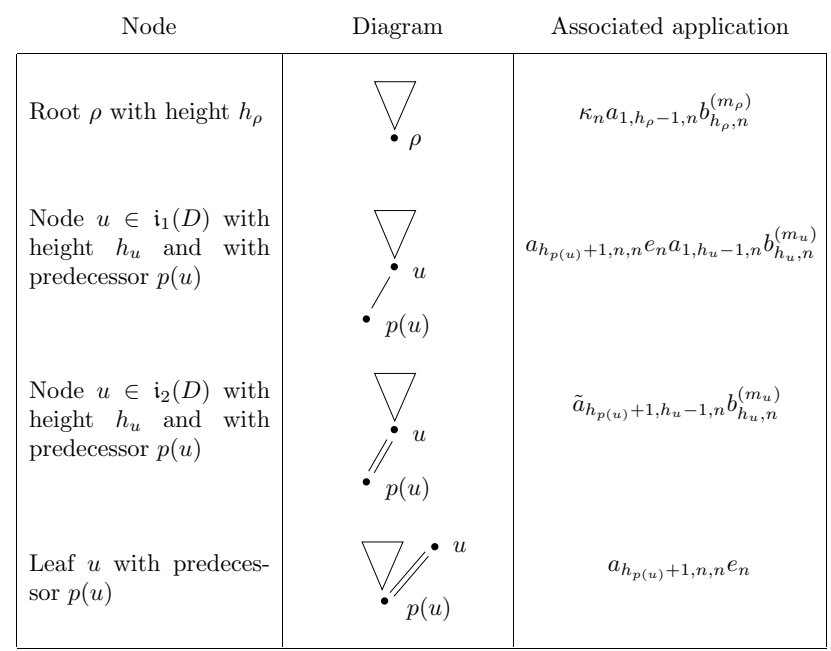

TABLE 1. Elementary applications associated with the different nodes of a diagram

3.1.1.6. Examples. - The following examples show how the notations are used.

The second order derivative of $\Psi_{n}$ is:

$$
D^{2} \Psi_{n}(z)=\sum_{k=1}^{n} \kappa_{n}(z) a_{1, k-1, n}(z) b_{k, n}^{(2)}(z)\left(a_{k+1, n, n}(z) e_{n}(z), a_{k+1, n, n}(z) e_{n}(z)\right)
$$

Thus

$$
D^{2} \Psi_{n}(z)=\sum_{h=1}^{n} f_{n}(U ; h)(z)=F_{n}(U)(z)
$$

where $U$ is the diagram with two leaves represented in Figure 2.

The third order derivative of $\Psi_{n}$ is:

$$
\begin{aligned}
D^{3} \Psi_{n} & =\sum_{k=1}^{n} \kappa_{n} a_{1, k-1, n} b_{k, n}^{(3)}\left(a_{k+1, n, n} e_{n}\right)^{\otimes 3} \\
& +3 \sum_{k=1}^{n} \sum_{\ell=1}^{k-1} \kappa_{n} a_{1, \ell-1, n} b_{\ell, n}^{(2)}\left(\tilde{a}_{\ell+1, k-1, n} b_{k, n}^{(2)}\left(a_{k+1, n, n} e_{n}\right)^{\otimes 2}, a_{\ell+1, n, n} e_{n}\right) \\
& -3 \sum_{k=1}^{n} \sum_{\ell=k}^{n} \kappa_{n} a_{1, \ell-1, n} b_{\ell, n}^{(2)}\left(a_{\ell+1, n, n} e_{n} a_{1, k-1, n} b_{k, n}^{(2)}\left(a_{k+1, n, n} e_{n}\right)^{\otimes 2}, a_{\ell+1, n, n} e_{n}\right)
\end{aligned}
$$

BULLETIN DE LA SOCiÉTÉ MATHÉMATIQUe DE FRANCE 
Therefore,

$$
D^{3} \Psi_{n}=F_{n}\left(D_{1}\right)+3 F_{n}\left(D_{2}\right)-3 F_{n}\left(D_{3}\right)
$$

where $D_{1}, D_{2}$ and $D_{3}$ are the diagrams with three leaves represented in Figure 2.
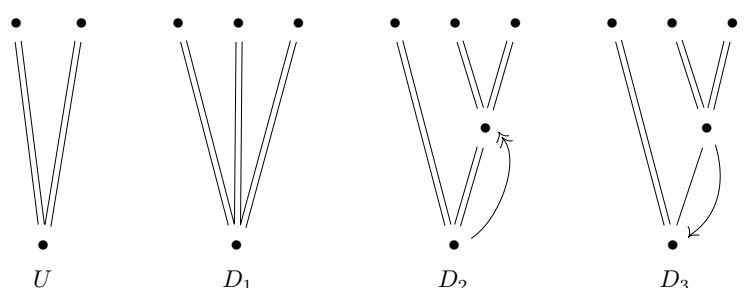

FIGURE 2. Diagrams of the second and third derivatives of $\Psi_{n}$

The following proposition gives an expression of the derivatives of $\Psi_{n}$ with the help of diagrams: the $k$-th derivative of $\Psi_{n}$ can be written using only maps associated with diagrams of $\mathcal{D}$ having $k$ leaves.

Proposition 3.1. - Let $k \in \mathbb{N} \backslash\{0,1\}$. The $k$-th derivative of $\Psi_{n}$ is a linear combination (independent of $n$ and of the choice of the applications $\varphi_{i}$ ) of maps $F_{n}(D)$ associated with a diagram $D=\left(X, E_{1}, E_{2}, I_{1}, I_{2}\right) \in \mathcal{D}$ having the following properties:

(i) $\left(X, E_{1} \cup E_{2}\right)$ is a tree having $k$ leaves;

(ii) $I_{1}$ and $I_{2}$ contain pairs of interior nodes and satisfy:

$\triangleright x \in \mathfrak{i}_{1}(D) \Leftrightarrow(x, p(x)) \in I_{1} ;$

$\triangleright x \in \mathfrak{i}_{2}(D) \Leftrightarrow(p(x), x) \in I_{2}$.

Expressions of the derivatives of $\Psi_{n}$ can be computed by induction with the following two assertions:

1) $D^{2} \Psi_{n}=F_{n}(U)$ where $U \in \mathcal{D}$ is the diagram with two leaves represented in Figure 2.

2) The expression of $D^{k} \Psi_{n}$ can be deduced from the expression of $D^{k-1} \Psi_{n}$ by replacing each term $F_{n}(D)$ in the expression of $D^{k-1} \Psi_{n}$ by:

$$
\begin{aligned}
& F_{n}\left(\mathfrak{t}_{1}(D ; \rho(D))\right)+F_{n}\left(\mathfrak{t}_{3}(D ; \rho(D))\right)-F_{n}\left(\mathfrak{t}_{4}(D ; \rho(D))\right) \\
& \quad+\sum_{u \in \ell(D)}\left(F_{n}\left(\mathfrak{t}_{2}(D ; u)\right)-F_{n}\left(\mathfrak{t}_{3}(D ; u)\right)\right) \\
& \quad+\sum_{u \in \mathfrak{i}_{1}(D)}\left(F_{n}\left(\mathfrak{t}_{1}(D ; u)\right)+F_{n}\left(\mathfrak{t}_{2}(D ; u)\right)-F_{n}\left(\mathfrak{t}_{3}(D ; u)\right)+F_{n}\left(\mathfrak{t}_{5}(D ; u)\right)\right) \\
& \quad+\sum_{u \in \mathfrak{i}_{2}(D)}\left(F_{n}\left(\mathfrak{t}_{1}(D ; u)\right)+F_{n}\left(\mathfrak{t}_{2}(D ; u)\right)-F_{n}\left(\mathfrak{t}_{3}(D ; u)\right)-F_{n}\left(\mathfrak{t}_{4}(D ; u)\right)\right)
\end{aligned}
$$

TOME $129-2001-\mathrm{N}^{\mathrm{O}} 3$ 
where $\mathfrak{t}_{i}$ for $i \in\{1, \ldots, 5\}$ are the transformations on the equivalence classes of diagrams represented in Figures 3 to 6.

The subset of diagrams $D \in \mathcal{D}$ having $k$ leaves and satisfying property (ii) is denoted by $\mathcal{T}_{k}$.

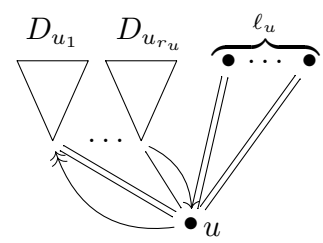

Diagram $D$

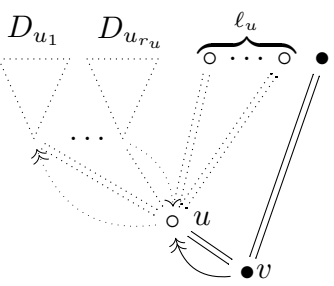

Diagram $\mathfrak{t}_{3}(D ; u)$

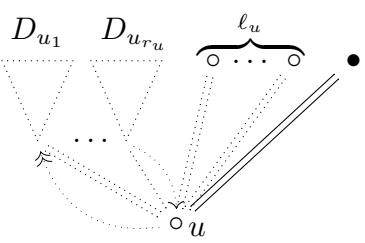

Diagram $\mathfrak{t}_{1}(D ; u)$

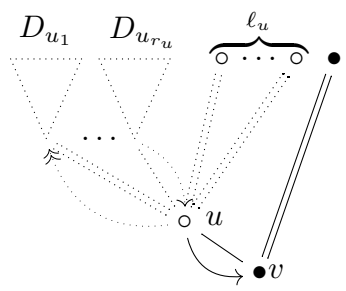

Diagram $\mathfrak{t}_{4}(D ; u)$

FiguRE 3. Transformations at the root $u$

3.1.2. Proof of Proposition 3.1. - The proof proceeds by induction on the order of the derivative of $\Psi_{n}$. Formulas (14) and (15) show that the proposition is true for $k=2$ and $k=3$.

Let $k$ be an integer greater than 1. Assume that Proposition 3.1 is true for the $k$-th derivative of $\Psi_{n}$. To prove the proposition, it suffices to show that, if $D \in \mathcal{T}_{k}$, then the derivative of the associated application $F_{n}(D)$ is a linear combination (whose coefficients do not depend on $n$ ) of terms like $F_{n}(\tilde{D})$, where $\tilde{D}$ is a diagram belonging to $\mathcal{T}_{k+1}$.

3.1.2.1. Derivatives of applications associated with a node. - The derivative of the application $F_{n}(D)$ associated with a diagram $D \in \mathcal{D}$, can be obtained by going all over the nodes of $D$ and summing all the terms that arise from the derivatives of applications associated with each node, that is:

- $\kappa_{n} a_{1, h_{u}-1, n} b_{h_{u}, n}^{\left(m_{u}\right)}$, if $u$ is the root of the tree $\left(X, E_{1} \cup E_{2}\right)$;

- $a_{h_{p(u)}+1, n, n} e_{n} a_{1, h_{u}-1, n} b_{h_{u}, n}^{\left(m_{u}\right)}$, if $u$ is a node belonging to $\mathfrak{i}_{1}(D)$;

BULLETIN DE LA SOCIÉTÉ MATHÉMATIQUe DE FRANCE 
- $\tilde{a}_{h_{p(u)}+1, h_{u}-1, n} b_{h_{u}, n}^{\left(m_{u}\right)}$, if $u$ is a node belonging to $\mathfrak{i}_{2}(D)$;

- $a_{h_{p(u)}+1, n, n} e_{n}$, if $u$ is a leaf of the node $p(u)$.

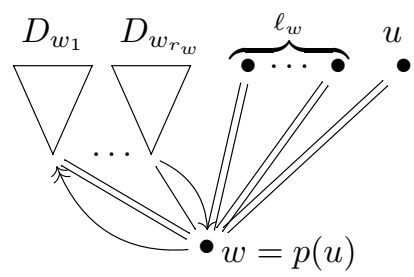

Diagram $D$

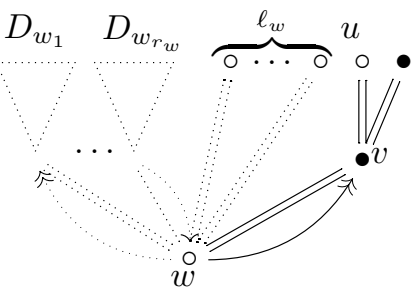

Diagram $\mathfrak{t}_{2}(D ; u)$

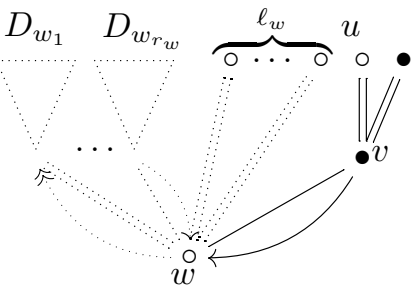

Diagram $\mathfrak{t}_{3}(D ; u)$

FiguRE 4. Transformations of the diagram $D$ at a leaf $u$

The following four lemmas give the result of the derivatives of these four types of applications. The derivatives of the elementary applications $a_{i, j, n}$, $b_{j, n}^{(m)}, e_{n}$ and $\kappa_{n}$ have been already computed (Formulas $(7),(8),(9)$ and (10)).

Lemma 3.2. - Let $j \in \mathbb{N}^{*}, m \in \mathbb{N} \backslash\{0,1\}$ and $\underline{v}=\left(v_{1}, \ldots, v_{m}\right) \in\left(\mathbb{R}^{N}\right)^{m}$. The derivative of the application $z \mapsto \kappa_{n}(z) a_{1, j-1, n}(z) b_{j, n}^{(m)}(z)(\underline{v})$ is

$$
\begin{aligned}
& D\left(\kappa_{n} a_{1, j-1, n} b_{j, n}^{(m)}(\underline{v})\right)=\kappa_{n} a_{1, j-1, n} b_{j, n}^{(m+1)}\left(\underline{v}, a_{j+1, n, n} e_{n}\right) \\
& +\sum_{\ell=1}^{j-1} \kappa_{n} a_{1, \ell-1, n} b_{\ell, n}^{(2)}\left(\tilde{a}_{\ell+1, j-1, n} b_{j, n}^{(m)}(\underline{v}), a_{\ell+1, n, n} e_{n}\right) \\
& \quad-\sum_{\ell=j}^{n} \kappa_{n} a_{1, \ell-1, n} b_{\ell, n}^{(2)}\left(a_{\ell+1, n, n} e_{n} a_{1, j-1, n} b_{j, n}^{(m)}(\underline{v}), a_{\ell+1, n, n} e_{n}\right) .
\end{aligned}
$$

TOME $129-2001-\mathrm{N}^{\mathrm{O}} 3$ 


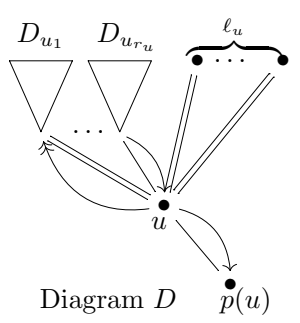

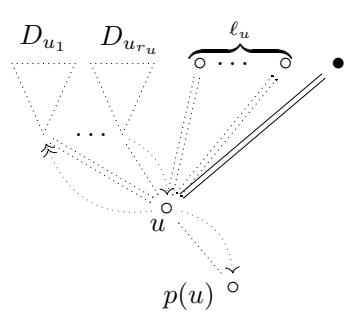

Diagram $\mathfrak{t}_{1}(D ; u)$

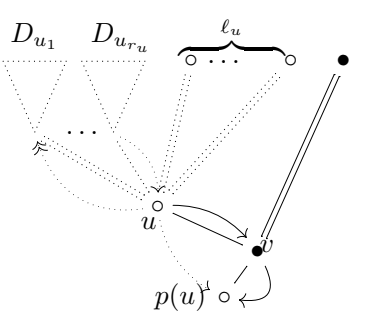

Diagram $\mathfrak{t}_{3}(D ; u)$

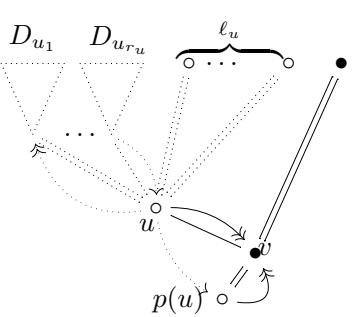

Diagram $\mathfrak{t}_{2}(D ; u)$

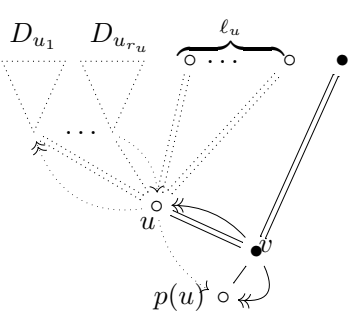

Diagram $\mathfrak{t}_{5}(D ; u)$

FiguRE 5. Transformations of the diagram $D$ at a node $u \in \mathfrak{i}_{1}(D)$

LemmA 3.3. - Let $v \in \mathbb{R}^{N}$ and $j \in \mathbb{N}^{*}$. The derivative of the application $z \mapsto a_{j, n, n}(z) e_{n}(z)(v)$ is

$$
\begin{aligned}
D\left(a_{j, n, n} e_{n}(v)\right)= & \sum_{\ell=j+1}^{n} \tilde{a}_{j, \ell-1, n} b_{\ell, n}^{(2)}\left(a_{\ell+1, n, n} e_{n}(v), a_{\ell+1, n, n} e_{n}\right) \\
& -\sum_{\ell=1}^{j} a_{j, n, n} e_{n} a_{1, \ell-1, n} b_{\ell, n}^{(2)}\left(a_{\ell+1, n, n} e_{n}(v), a_{\ell+1, n, n} e_{n}\right)
\end{aligned}
$$

BULletin DE LA SOCiÉtÉ MATHÉmATiQUe DE FRANCE 


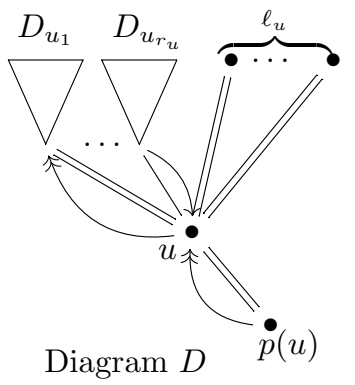

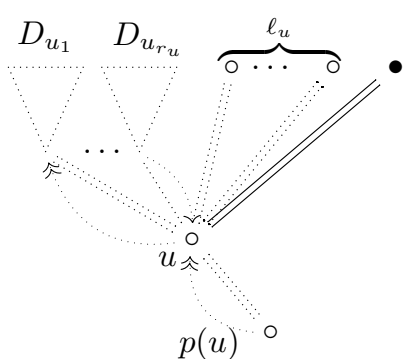

Diagram $\mathfrak{t}_{1}(D ; u)$

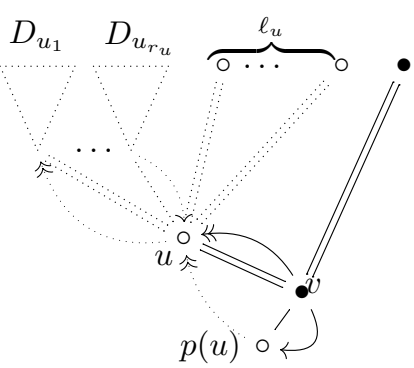

Diagram $\mathfrak{t}_{3}(D ; u)$

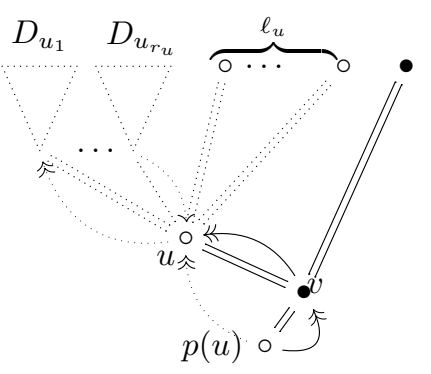

Diagram $\mathfrak{t}_{2}(D ; u)$

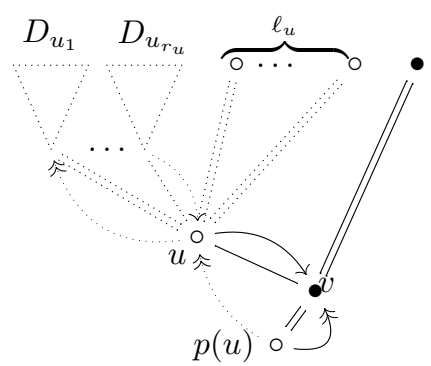

Diagram $\mathfrak{t}_{4}(D ; u)$

Figure 6 . Transformations of the diagram $D$ at a node $u \in \mathfrak{i}_{2}(D)$

LEMMA 3.4. - Let $i, j \in \mathbb{N}^{*}$ such that $i<j, m \in \mathbb{N} \backslash\{0,1\}$ and let $\underline{v}=$ $\left(v_{1}, \ldots, v_{m}\right) \in\left(\mathbb{R}^{N}\right)^{m}$. The derivative of the application

$$
z \longmapsto a_{j, n, n}(z) e_{n}(z) a_{1, i-1, n}(z) b_{i, n}^{(m)}(z)(\underline{v})
$$

TOME $129-2001-\mathrm{N}^{\mathrm{O}} 3$ 
is

$$
\begin{aligned}
& D\left(a_{j, n, n} e_{n} a_{1, i-1, n} b_{i, n}^{(m)}(\underline{v})\right)=a_{j, n, n} e_{n} a_{1, i-1, n} b_{i, n}^{(m+1)}\left(\underline{v}, a_{i+1, n, n} e_{n}\right) \\
& \quad+\sum_{\ell=j}^{n} \tilde{a}_{j, \ell-1, n} b_{\ell, n}^{(2)}\left(a_{\ell+1, n, n} e_{n} a_{1, i-1, n} b_{i, n}^{(m)}(\underline{v}), a_{\ell+1, n, n} e_{n}\right) \\
& \quad-\sum_{\ell=i}^{j-1} a_{j, n, n} e_{n} a_{1, \ell-1, n} b_{\ell, n}^{(2)}\left(a_{\ell+1, n, n} e_{n} a_{1, i-1, n} b_{i, n}^{(m)}(\underline{v}), a_{\ell+1, n, n} e_{n}\right) \\
& \quad+\sum_{\ell=1}^{i-1} a_{j, n, n} e_{n} a_{1, \ell-1, n} b_{\ell, n}^{(2)}\left(\tilde{a}_{\ell+1, i-1, n} b_{i, n}^{(m)}(\underline{v}), a_{\ell+1, n, n} e_{n}\right)
\end{aligned}
$$

Proof. - For all $i, j \in \mathbb{N}$ such that $0<i<j$ :

$$
\begin{aligned}
& D\left(a_{j, n, n} e_{n} a_{1, i, n}(u)\right)=\sum_{\ell=j}^{n} a_{j, \ell-1, n} b_{\ell, n}^{(2)}\left(a_{\ell+1, n, n} e_{n} a_{1, i, n}(u), a_{\ell+1, n, n} e_{n}\right) \\
& -\sum_{\ell=1}^{n} a_{j, n, n} e_{n} a_{1, \ell-1, n} b_{\ell, n}^{(2)}\left(a_{\ell+1, n, n} e_{n} a_{1, i, n}(u), a_{\ell+1, n, n} e_{n}\right) \\
& +\sum_{\ell=1}^{i} a_{j, n, n} e_{n} a_{1, \ell-1, n} b_{\ell, n}^{(2)}\left(a_{\ell+1, i, n}(u), a_{\ell+1, n, n} e_{n}\right)
\end{aligned}
$$

Replacing every term like $a_{p, q, n}$ with $1<p \leq q$ by $\tilde{a}_{p, q, n}+a_{p, n, n} e_{n} a_{1, q, n}$ gives:

$$
\begin{aligned}
& D\left(a_{j, n, n} e_{n} a_{1, i, n}(u)\right)=\sum_{\ell=j}^{n} \tilde{a}_{j, \ell-1, n} b_{\ell, n}^{(2)}\left(a_{\ell+1, n, n} e_{n} a_{1, i, n}(u), a_{\ell+1, n, n} e_{n}\right) \\
& -\sum_{\ell=i+1}^{j-1} a_{j, n, n} e_{n} a_{1, \ell-1, n} b_{\ell, n}^{(2)}\left(a_{\ell+1, n, n} e_{n} a_{1, i, n}(u), a_{\ell+1, n, n} e_{n}\right) \\
& +\sum_{\ell=1}^{i} a_{j, n, n} e_{n} a_{1, \ell-1, n} b_{\ell, n}^{(2)}\left(\tilde{a}_{\ell+1, i, n}(u), a_{\ell+1, n, n} e_{n}\right)
\end{aligned}
$$

LEMma 3.5. - Let $i, j \in \mathbb{N}^{*}$ such that $i<j, m \in \mathbb{N} \backslash\{0,1\}$ and let $\underline{v}=$ $\left(v_{1}, \ldots, v_{m}\right) \in\left(\mathbb{R}^{N}\right)^{m}$. The derivative of the application

$$
z \longmapsto \tilde{a}_{i, j-1, n}(z) b_{j, n}^{(m)}(z)(\underline{v})
$$

BULLETIN DE LA SOCiÉTÉ MATHÉMATIQUe DE FRANCE 
is

$$
\begin{aligned}
& D\left(\tilde{a}_{i, j-1, n} b_{j, n}^{(m)}(\underline{v})\right)=\tilde{a}_{i, j-1, n} b_{j, n}^{(m+1)}\left(\underline{v}, a_{j+1, n, n} e_{n}\right) \\
& +\sum_{\ell=i}^{j-1} \tilde{a}_{i, j-1, n} b_{\ell, n}^{(2)}\left(\tilde{a}_{\ell+1, j-1, n} b_{j, n}^{(m)}(\underline{v}), a_{\ell+1, n, n} e_{n}\right) \\
& -\sum_{\ell=1}^{i-1} a_{i, n, n} e_{n} a_{1, \ell-1, n} b_{\ell, n}^{(2)}\left(\tilde{a}_{\ell+1, j-1, n} b_{j, n}^{(m)}(\underline{v}), a_{\ell+1, n, n} e_{n}\right) \\
& -\sum_{\ell=j}^{n} \tilde{a}_{i, \ell-1, n} b_{\ell, n}^{(2)}\left(a_{\ell+1, n, n} e_{n} a_{1, j-1, n} b_{j, n}^{(m)}(\underline{v}), a_{\ell+1, n, n} e_{n}\right)
\end{aligned}
$$

Proof. — The formulas (7), (8) and (9) of the derivatives of the elementary applications give:

$$
\begin{aligned}
& D\left(\tilde{a}_{i, j, n}(v)\right)=\sum_{\ell=i}^{j} a_{i, \ell-1, n} b_{\ell, n}^{(2)}\left(a_{\ell+1, j, n}(v), a_{\ell+1, n, n} e_{n}\right) \\
& -\sum_{\ell=i}^{n} a_{i, \ell-1, n} b_{\ell, n}^{(2)}\left(a_{\ell+1, n, n} e_{n} a_{1, j, n}(v), a_{\ell+1, n, n} e_{n}\right) \\
& +\sum_{\ell=1}^{n} a_{i, n, n} e_{n} a_{1, \ell-1, n} b_{\ell, n}^{(2)}\left(a_{\ell+1, n, n} e_{n} a_{1, j, n}(v), a_{\ell+1, n, n} e_{n}\right) \\
& -\sum_{\ell=1}^{j} a_{i, n, n} e_{n} a_{1, \ell-1, n} b_{\ell, n}^{(2)}\left(a_{\ell+1, j, n}(v), a_{\ell+1, n, n} e_{n}\right)
\end{aligned}
$$

The first two terms and the last two terms can be reorganized:

$$
\begin{aligned}
& D\left(\tilde{a}_{i, j, n}(v)\right)=\sum_{\ell=i}^{j} a_{i, \ell-1, n} b_{\ell, n}^{(2)}\left(\tilde{a}_{\ell+1, j, n}(v), a_{\ell+1, n, n} e_{n}\right) \\
& -\sum_{\ell=j+1}^{n} a_{i, \ell-1, n} b_{\ell, n}^{(2)}\left(a_{\ell+1, n, n} e_{n} a_{1, j, n}(v), a_{\ell+1, n, n} e_{n}\right) \\
& +\sum_{\ell=j+1}^{n} a_{i, n, n} e_{n} a_{1, \ell-1, n} b_{\ell, n}^{(2)}\left(a_{\ell+1, n, n} e_{n} a_{1, j, n}(v), a_{\ell+1, n, n} e_{n}\right) \\
& -\sum_{\ell=1}^{j} a_{i, n, n} e_{n} a_{1, \ell-1, n} b_{\ell, n}^{(2)}\left(\tilde{a}_{\ell+1, j, n}(v), a_{\ell+1, n, n} e_{n}\right)
\end{aligned}
$$

TOME $129-2001-\mathrm{N}^{\mathrm{O}} 3$ 
Rearranging the first term with the last term on one hand, and the second with the third on the other hand, yields:

$$
\begin{aligned}
& D\left(\tilde{a}_{i, j, n}(v)\right)=\sum_{\ell=i}^{j} \tilde{a}_{i, \ell-1, n} b_{\ell, n}^{(2)}\left(\tilde{a}_{\ell+1, j, n}(v), a_{\ell+1, n} e_{n}\right) \\
& -\sum_{\ell=1}^{i-1} a_{i, n, n} e_{n} a_{1, \ell-1, n} b_{\ell, n}^{(2)}\left(\tilde{a}_{\ell+1, j, n}(v), a_{\ell+1, n, n} e_{n}\right) \\
& -\sum_{\ell=j+1}^{n} \tilde{a}_{i, \ell-1, n} b_{\ell, n}^{(2)}\left(a_{\ell+1, n, n} e_{n} a_{1, j, n}(v), a_{\ell+1, n, n} e_{n}\right)
\end{aligned}
$$

3.1.2.2. Derivative of an application associated with a diagram. — Summing the terms provided by the derivative of the application associated with each node of a diagram $D \in \mathcal{T}_{k}$, leads to the following expression of the derivative of $F_{n}(D)$ :

$$
\begin{aligned}
& F_{n}^{\prime}(D)=F_{n}(D(1 ; \rho(D)))+F_{n}(D(3 ; \rho(D)))-F_{n}(D(4 ; \rho(D))) \\
& +\sum_{u \in \ell(D)}\left(F_{n}(D(2 ; u))-F_{n}(D(3 ; u))\right) \\
& +\sum_{u \in \mathfrak{i}_{1}(D)}\left(F_{n}(D(1 ; u))+F_{n}(D(2 ; u))-F_{n}(D(3 ; u))+F_{n}(D(5 ; u))\right) \\
& +\sum_{u \in \mathfrak{i}_{2}(D)}\left(F_{n}(D(1 ; u))+F_{n}(D(2 ; u))-F_{n}(D(3 ; u))-F_{n}(D(4 ; u))\right)
\end{aligned}
$$

where $D(i ; u)$ for $i \in\{1, \ldots, 5\}$ designate the diagrams obtained by a transformation at the node $u$ of $D$. More precisely, $D(i ; u)$ is the diagram which corresponds to the term numbered $[i]$ in Lemmas $3.2,3.4,3.5$ or 3.3 . It is the result of the derivative of the application associated with the node $u$. The numbers are chosen in order to group the terms obtained by a given type of transformation on the diagram $D$.

REMARK 3.6. - For every $i \in\{1, \ldots, 5\}$, the image of the equivalence class of the diagram $D$ by the transformation $\mathfrak{t}_{i}$ applied at the node $u$ is equal to the equivalence class of the diagram $D(i ; u)$.

The comparison of the new diagrams $D(i ; u)$ with the diagram $D$ allows to check that each diagram $D(i ; u)$ belongs to $\mathcal{T}_{k+1}$, if $D$ lies in $\mathcal{T}_{k}$. This ends the proof of Proposition 3.1.

BULletin DE LA SOCiÉtÉ MATHÉmATiQUe DE FRANCE 
3.2. Convergence of the derivatives of $\boldsymbol{\Upsilon}_{\boldsymbol{n}}$ at zero. - This subsection is devoted to studying the asymptotic behaviour of $\left(D^{k} \Upsilon_{n}(0)\right)_{n}$. The notations are those used for the case 1 in Subsection 3.1; namely with $\varphi_{i}=\phi\left(\theta^{-i}\right)$ for every $i \in\{1, \ldots, n\}$. At point 0 , the applications associated with diagrams do not depend on $n$, thus $n$ is omitted in the notations.

By Proposition 3.1, to prove the convergence result for $\left(D^{k} \Upsilon_{n}(0)\right)_{n}$, it suffices to establish the following lemma:

LEMMA 3.7. - Let $k \geq 2$. Assume that $\log ^{+}\left\|B^{(j)}\right\| \in L^{1}(\Omega, \mathbb{P})$ for each $j \in\{1, \ldots, k\}$. If $\lambda_{s+1}-k \lambda_{s}<0$, then the sequence $\left(\sum_{h \in H_{n}(D)} f(D ; h)(0)\right)_{n}$ converges $\mathbb{P}$-almost surely, for every $D \in \mathcal{T}_{k}$.

Using Lemma 3.7 and Proposition 3.1, it is possible to compute the limit of the sequence $\left(D^{k} \Upsilon_{n}(0)\right)$ :

Proposition 3.8. - Let $k \geq 2$. Assume that $\log ^{+}\left\|A^{ \pm 1}\right\|$ and $\log ^{+}\left\|B^{(j)}\right\|$, for $j \in\{2, \ldots, k\}$, lie in $L^{1}(\Omega, \mathbb{P})$. Furthermore, assume that $\lambda_{s+1}-k \lambda_{s}<0$.

1) The first $k$ derivatives of $\Upsilon_{n}$ at 0 converge $\mathbb{P}$-almost surely as $n$ tends to $+\infty$.

2) Set $F(D)=\sum_{h \in H(D)} f(D ; h)(0)$ for $D \in \bigcup_{j=2}^{k} \mathcal{T}_{j}$. For $j \in\{2, \ldots, k\}$, the limit $S^{(j)}$ of $\left(D^{j} \Upsilon_{n}(0)\right)_{n}$ can be determined by induction using the following assertions:

- $S^{(2)}=F(U)$ where $U$ is the diagram in $\mathcal{D}$ having two leaves (Fig. 2);

- for $j \in\{3, \ldots, k\}$, the expression of $S^{(j)}$ can be deduced from the expression of $S^{(j-1)}$ by replacing each application $F(D)$ in $S^{(j-1)}$ by

$$
\begin{aligned}
& F\left(\mathfrak{t}_{1}(D ; \rho(D))\right)+F\left(\mathfrak{t}_{3}(D ; \rho(D))\right)-F\left(\mathfrak{t}_{4}(D ; \rho(D))\right) \\
& +\sum_{u \in \ell(D)}\left(F\left(\mathfrak{t}_{2}(D ; u)\right)-F\left(\mathfrak{t}_{3}(D ; u)\right)\right) \\
& +\sum_{u \in \mathfrak{i}_{1}(D)}\left(F\left(\mathfrak{t}_{1}(D ; u)\right)+F\left(\mathfrak{t}_{2}(D ; u)\right)-F\left(\mathfrak{t}_{3}(D ; u)\right)+F\left(\mathfrak{t}_{5}(D ; u)\right)\right) \\
& +\sum_{u \in \mathfrak{i}_{2}(D)}\left(F\left(\mathfrak{t}_{1}(D ; u)\right)+F\left(\mathfrak{t}_{2}(D ; u)\right)-F\left(\mathfrak{t}_{3}(D ; u)\right)-F\left(\mathfrak{t}_{4}(D ; u)\right)\right)
\end{aligned}
$$

where $\mathfrak{t}_{i}$ for $i \in\{1, \ldots, 5\}$ are the transformations on the equivalence classes of diagrams represented Figures 3 to 6.

The proof of Lemma 3.7 begins by calculating an upper bound for the norm of the applications associated with the diagrams of $\mathcal{D}$ and taken at point 0 .

TOME $129-2001-\mathrm{N}^{\mathrm{O}} 3$ 
The upper bound follows directly from the inequalities established in Section 2 (Lemmas 2.7,2.8, 2.10 and 2.11): for all $n \in \mathbb{N}^{*}$,

(16) $\forall j \in\{1, \ldots, n\}, m \in\{2, \ldots, k\},\left\|b_{j, n}^{(m)}(0)\right\|=\left\|B^{(m)}\left(\theta^{-j}\right)\right\| \leq C_{\epsilon}^{(m)} \mathrm{e}^{j \epsilon}$,

(17) $\forall j, 1 \leq j \leq n,\left\|\kappa_{n}(0) a_{1, j, n}(0)\right\|=\left\|(\mathrm{I}-\Pi) A_{j}\left(\theta^{-j}\right)\right\| \leq C_{\epsilon} \mathrm{e}^{j\left(\lambda_{s+1}+\epsilon\right)}$,

(18) $\forall j, 0 \leq j \leq n,\left\|a_{j+1, n, n}(0) e_{n}(0)\right\|=\left\|A_{-j} \Pi\right\| \leq C_{\epsilon} \mathrm{e}^{j\left(-\lambda_{s}+\epsilon\right)}$,

(19) $\forall i, 0<i \leq j \leq n,\left\|\tilde{a}_{i+1, j, n}(0)\right\|=\left\|\tilde{A}_{i, j}\right\| \leq C_{\epsilon} \mathrm{e}^{(j-i) \lambda_{s+1}+(j+i) \epsilon}$, $\forall i, 0<j \leq i \leq n,\left\|a_{i+1, n, n}(0) e_{n}(0) a_{1, j, n}(0)\right\|=\left\|A_{-i} \Pi A_{j}\left(\theta^{-j}\right)\right\|$

$$
\leq C_{\epsilon} \mathrm{e}^{(j-i) \lambda_{s}+(i+j) \epsilon},
$$

where $C_{\epsilon}$ and $C_{\epsilon}^{(m)}$ for all $2 \leq m \leq k$, are almost surely finite random variables. Actually, two upper bounds are given in the following lemma, the first will allow to prove Lemma 3.7 when $\lambda_{s} \leq 0$ and the second when $\lambda_{s}>0$ :

LEMMA 3.9. - Let $\epsilon>0$ and $D=\left(X, E_{1}, E_{2}, I_{1}, I_{2}\right)$ be a diagram in $\bigcup_{i=2}^{k} \mathcal{T}_{i}$.

1) There exists an almost surely finite random variable $C_{\epsilon}(D)$, such that for all $h \in H(D)$ :

$$
\|f(D ; h)(0)\| \leq C_{\epsilon}(D) \exp \left(\sum_{u \in \mathfrak{i}(D)} h_{u} \alpha_{u}\right)
$$

where $\left(\alpha_{u}\right)_{u \in \mathfrak{i}(D)}$ is a family of reals defined by:

$$
\alpha_{u}=\left\{\begin{array}{c}
-\lambda_{s}\left(\ell_{u}+r_{u}^{(1)}\right)+\left(1-r_{u}^{(2)}\right) \lambda_{s+1}+\epsilon\left(2+m_{u}\right) \\
\text { if } u \in \mathfrak{i}_{2}(D) \cup\{\rho(D)\}, \\
\lambda_{s}\left(1-\ell_{u}-r_{u}^{(1)}\right)-r_{u}^{(2)} \lambda_{s+1}+\epsilon\left(2+m_{u}\right) \\
\text { if } u \in \mathfrak{i}_{1}(D) .
\end{array}\right.
$$

2) There exists an almost surely finite random variable $C_{\epsilon}(D)$, such that for all $h \in H(D)$ :

$$
\|f(D ; h)(0)\| \leq C_{\epsilon}(D) \exp \left(\sum_{u \in \mathfrak{i}(D)} h_{u}\left(\lambda_{s}\left(1-m_{u}\right)+\epsilon\left(2+m_{u}\right)\right)\right)
$$

Proof. - Let us begin by establishing that for every $u \in \mathfrak{i}(D)^{*}$ and $h \in H(D)$, the following inequality holds:

$$
\begin{aligned}
& \left\|f_{h_{p(u)}}\left(D_{u} ; h_{\mid D_{u}}\right)(0)\right\| \leq C_{\epsilon}\left(D_{u}\right) \exp \left(\sum_{v \in \mathfrak{i}\left(D_{u}\right)} h_{v} \alpha_{v}\right) \\
& \quad \exp \left(h_{p(u)}\left(-\lambda_{s} 1_{\left\{u \in \mathfrak{i}_{1}(D)\right\}}-\lambda_{s+1} 1_{\left\{u \in \mathfrak{i}_{2}(D)\right\}}+\epsilon\right)+\sum_{v \in \mathfrak{i}\left(D_{u}\right)} h_{v} \alpha_{v}\right)
\end{aligned}
$$

where $C_{\epsilon}\left(D_{u}\right)=\prod_{v \in \mathfrak{i}\left(D_{u}\right)}\left(C_{\epsilon}^{1+\ell_{v}} C_{\epsilon}^{\left(m_{v}\right)}\right) \exp \left(-\lambda_{s+1}\left(r\left(D_{u}\right)+1\right)\right)$. 
The proof is by induction on the number of interior nodes of $D_{u}$ :

1) Assume that $\mathfrak{i}\left(D_{u}\right)=\{u\}$ (in this case, $\ell_{u}=m_{u}$ ). If $u \in \mathfrak{i}_{1}(D)$, then $h_{u} \leq h_{p(u)}$ and

$$
f_{h_{p(u)}}\left(D_{u} ; h_{\mid D_{u}}\right)(0)=A_{-h_{p(u)}} \Pi A_{h_{u}-1}\left(\theta^{-\left(h_{u}-1\right)}\right) B^{\left(m_{u}\right)}\left(\theta^{-h_{u}}\right)\left(\left(A_{-h_{u}} \Pi\right)^{\otimes \ell_{u}}\right) .
$$

Thus, the upper bounds previously enumerated yield:

$$
\begin{aligned}
& \left\|f_{h_{p(u)}}\left(D_{u} ; h_{\mid D_{u}}\right)(0)\right\| \\
& \quad \leq\left\|A_{-h_{p(u)}} \Pi A_{h_{u}-1}\left(\theta^{-\left(h_{u}-1\right)}\right)\right\| \cdot\left\|B^{\left(m_{u}\right)}\left(\theta^{-h_{u}}\right)\right\| \cdot\left\|A_{-h_{u}} \Pi\right\|^{\ell_{u}} \\
& \quad \leq C_{\epsilon}^{1+\ell_{u}} C_{\epsilon}^{\left(m_{u}\right)} \mathrm{e}^{\left(h_{u}-1-h_{p(u)}\right) \lambda_{s}+\left(h_{u}-1+h_{p(u)}\right) \epsilon} \mathrm{e}^{h_{u} \epsilon+\ell_{u} h_{u}\left(-\lambda_{s}+\epsilon\right)} \\
& \quad \leq C_{\epsilon}\left(D_{u}\right) \mathrm{e}^{h_{p(u)}\left(-\lambda_{s}+\epsilon\right)+h_{u}\left(\lambda_{s}\left(1-\ell_{u}\right)+\epsilon\left(2+\ell_{u}\right)\right)}
\end{aligned}
$$

If $u \in \mathfrak{i}_{2}(D)$, then

$$
f_{h_{p(u)}}\left(D_{u} ; h_{\mid D_{u}}\right)(0)=\tilde{A}_{h_{p(u)}, h_{u}-1} B^{\left(m_{u}\right)}\left(\theta^{-h_{u}}\right)\left(\left(A_{-h_{u}} \Pi\right)^{\otimes \ell_{u}}\right) .
$$

Thus,

$$
\begin{aligned}
& \left\|f_{h_{p(u)}}\left(D_{u} ; h_{\mid D_{u}}\right)(0)\right\| \\
& \quad \leq\left\|\tilde{A}_{h_{p(u)}, h_{u}-1}\right\| \cdot\left\|B^{\left(m_{u}\right)}\left(\theta^{-h_{u}}\right)\right\| \cdot\left\|A_{-h_{u}} \Pi\right\|^{\ell_{u}} \\
& \quad \leq C_{\epsilon}^{1+\ell_{u}} C_{\epsilon}^{\left(m_{u}\right)} \mathrm{e}^{\left(h_{u}-1-h_{p(u)}\right) \lambda_{s+1}+\left(h_{u}-1+h_{p(u)}\right) \epsilon} \mathrm{e}^{h_{u} \epsilon+\ell_{u} h_{u}\left(-\lambda_{s}+\epsilon\right)} \\
& \quad \leq C_{\epsilon}\left(D_{u}\right) \mathrm{e}^{h_{p(u)}\left(-\lambda_{s+1}+\epsilon\right)+h_{u}\left(\lambda_{s+1}-\ell_{u} \lambda_{s}+\epsilon\left(2+\ell_{u}\right)\right)}
\end{aligned}
$$

Therefore, the result holds if $u$ is the unique interior node of $D_{u}$.

2) Let $j \geq 1$. Assume now that the result holds for interior nodes $u$ of $D$ such that $D_{u}$ contains at most $j$ interior nodes. Consider a node $u$ of $D$ such that $\left|\mathfrak{i}\left(D_{u}\right)\right|=j+1$ and denote the non-terminal sons of $u$ by $u_{1}, \ldots, u_{r}$ :

- If $u \in \mathfrak{i}_{1}(D)$, then

$$
\begin{aligned}
f_{h_{p(u)}}\left(D_{u} ; h_{\mid D_{u}}\right)(0)= & A_{-h_{p(u)}} \Pi A_{h_{u}-1}\left(\theta^{-\left(h_{u}-1\right)}\right) \\
& B^{\left(m_{u}\right)}\left(\theta^{-h_{u}}\right)\left(f_{h_{u}}\left(D_{u_{1}} ; h_{\mid D_{u_{1}}}\right)(0)\right. \\
& \left.\ldots, f_{h_{u}}\left(D_{u_{r}} ; h_{\mid D_{u_{r}}}\right)(0),\left(A_{-h_{u}} \Pi\right)^{\otimes \ell_{u}}\right) .
\end{aligned}
$$

- If $u \in \mathfrak{i}_{2}(D)$, then

$$
\begin{array}{r}
f_{h_{p(u)}}\left(D_{u} ; h_{\mid D_{u}}\right)(0)=\tilde{A}_{h_{p(u)}, h_{u}-1} B^{\left(m_{u}\right)}\left(\theta^{-h_{u}}\right)\left(f_{h_{u}}\left(D_{u_{1}} ; h_{\mid D_{u_{1}}}\right)(0)\right. \\
\left.\ldots, f_{h_{u}}\left(D_{u_{r}} ; h_{\mid D_{u_{r}}}\right)(0),\left(A_{-h_{u}} \Pi\right)^{\otimes \ell_{u}}\right) .
\end{array}
$$

As $D_{u_{1}}, \ldots, D_{u_{r}}$ have at most $j$ interior nodes, the induction hypothesis can be applied to them:

TOME $129-2001-\mathrm{N}^{\mathrm{O}} 3$ 
- If $u \in \mathfrak{i}_{1}(D)$, then

$$
\begin{aligned}
&\left\|f_{h_{p(u)}}\left(D_{u} ; h_{\mid D_{u}}\right)(0)\right\| \leq C_{\epsilon}^{1+\ell_{u}} C_{\epsilon}^{\left(m_{u}\right)} \mathrm{e}^{\left(h_{u}-1-h_{p(u)}\right) \lambda_{s}+\left(h_{u}-1+h_{p(u)}\right) \epsilon} \mathrm{e}^{h_{u} \epsilon+\ell_{u} h_{u}\left(-\lambda_{s}+\epsilon\right)} \prod_{i=1}^{r}\left\|f_{h_{u}}\left(D_{u_{i}} ; h_{\mid D_{u_{i}}}\right)(0)\right\| \\
& \leq C_{\epsilon}^{1+\ell_{u}} C_{\epsilon}^{\left(m_{u}\right)} \mathrm{e}^{-\lambda_{s}} \mathrm{e}^{h_{p(u)}\left(-\lambda_{s}+\epsilon\right)+h_{u}\left(\lambda_{s}\left(1-\ell_{u}\right)+\epsilon\left(2+\ell_{u}\right)\right)} \\
& \prod_{i=1}^{r} C_{\epsilon}\left(D_{u_{i}}\right) \mathrm{e}^{h_{u}\left(r_{u}^{(1)}\left(-\lambda_{s}+\epsilon\right)+r_{u}^{(2)}\left(-\lambda_{s+1}+\epsilon\right)\right)} \\
& \exp \left(\sum_{v \in \mathfrak{i}\left(D_{u}\right) \backslash\{u\}} h_{v} \alpha_{v}\right) \\
& \leq C_{\epsilon}\left(D_{u}\right) \exp \left(h_{p(u)}\left(-\lambda_{s}+\epsilon\right)+\sum_{v \in \mathfrak{i}\left(D_{u}\right)} h_{v} \alpha_{v}\right) .
\end{aligned}
$$

- If $u \in \mathfrak{i}_{2}(D)$, then

$$
\begin{gathered}
\left\|f_{h_{p(u)}}\left(D_{u} ; h_{\mid D_{u}}\right)(0)\right\| \leq C_{\epsilon}^{1+\ell_{u}} C_{\epsilon}^{\left(m_{u}\right)} \mathrm{e}^{\left(h_{u}-1-h_{p(u)}\right) \lambda_{s+1}+\left(h_{u}-1+h_{p(u)}\right) \epsilon} \\
\mathrm{e}^{h_{u} \epsilon+\ell_{u} h_{u}\left(-\lambda_{s}+\epsilon\right)} \prod_{i=1}^{r}\left\|f_{h_{u}}\left(D_{u_{i}} ; h_{\mid D_{u_{i}}}\right)(0)\right\| \\
\leq C_{\epsilon}^{1+\ell_{u}} C_{\epsilon}^{\left(m_{u}\right)} \mathrm{e}^{-\lambda_{s+1}} \mathrm{e}^{h_{p(u)}\left(-\lambda_{s+1}+\epsilon\right)} \\
\mathrm{e}^{h_{u}\left(\lambda_{s+1}-\ell_{u} \lambda_{s}+\epsilon\left(2+\ell_{u}\right)\right)} \prod_{i=1}^{r} C_{\epsilon}\left(D_{u_{i}}\right) \\
\leq C_{\epsilon}\left(D_{u}\right) \exp \left(h_{p(u)}\left(-\lambda_{s+1}+\epsilon\right)+\sum_{v \in \mathfrak{i}\left(D_{u}\right)} h_{v} \alpha_{v}\right) .
\end{gathered}
$$

This closes the induction proof of inequality (22).

Let $\rho$ denote the root of $D$ and $\rho_{1}, \ldots, \rho_{r}$ the non-terminal sons of $\rho$. Then

$$
\begin{aligned}
f(D ; h)(0)=(\mathrm{I}-\Pi) A_{h_{\rho}-1}\left(\theta^{-\left(h_{\rho}-1\right)}\right) B^{\left(m_{\rho}\right)}\left(\theta^{-h_{\rho}}\right) \\
\\
\left(f_{h_{\rho}}\left(D_{\rho_{1}} ; h_{\mid D_{\rho_{1}}}\right)(0), \ldots, f_{h_{\rho}}\left(D_{\rho_{r}} ; h_{\mid D_{\rho_{r}}}\right)(0),\left(A_{-h_{\rho}} \Pi\right)^{\otimes \ell_{\rho}}\right) .
\end{aligned}
$$

Using that $\left\|(\mathrm{I}-\Pi) A_{h_{\rho}-1}\left(\theta^{-\left(h_{\rho}-1\right)}\right)\right\| \leq C_{\epsilon} \mathrm{e}^{\left(h_{\rho}-1\right)\left(\lambda_{s+1}+\epsilon\right)}$ gives:

$$
\begin{array}{r}
\left\|f\left(D_{\rho} ; h_{\mid D_{\rho}}\right)(0)\right\| \leq C_{\epsilon}^{1+\ell_{\rho}} C_{\epsilon}^{\left(m_{\rho}\right)} \mathrm{e}^{\left(h_{\rho}-1\right)\left(\lambda_{s+1}+\epsilon\right)+h_{\rho} \epsilon+\ell_{\rho} h_{\rho}\left(-\lambda_{s}+\epsilon\right)} \\
\prod_{i=1}^{r}\left\|f_{h_{\rho}}\left(D_{\rho_{i}} ; h_{\mid D_{\rho_{i}}}\right)(0)\right\|
\end{array}
$$

BULLETIN DE LA SOCIÉtÉ MATHÉMATIQUE DE FRANCE 


$$
\begin{aligned}
& \leq C_{\epsilon}^{1+\ell_{\rho}} C_{\epsilon}^{\left(m_{\rho}\right)} \mathrm{e}^{-\lambda_{s+1}} \mathrm{e}^{h_{\rho}\left(\lambda_{s+1}-\ell_{\rho} \lambda_{s}+\epsilon\left(2+\ell_{\rho}\right)\right)} \\
& \quad \prod_{i=1}^{r} C_{\epsilon}\left(D_{\rho_{i}}\right) \mathrm{e}^{h_{\rho}\left(r_{\rho}^{(1)}\left(-\lambda_{s}+\epsilon\right)+r_{\rho}^{(2)}\left(\lambda_{s+1}+\epsilon\right)\right)+\Sigma_{v \in \mathfrak{i}(D) \backslash\{\rho\}} h_{v} \alpha_{v}} \\
& \leq C_{\epsilon}(D) \exp \left(\sum_{v \in \mathfrak{i}(D)} h_{v} \alpha_{v}\right)
\end{aligned}
$$

where $C_{\epsilon}(D)=\prod_{v \in \mathfrak{i}(D)}\left(C_{\epsilon}^{1+\ell_{v}} C_{\epsilon}^{\left(m_{v}\right)}\right) \exp \left(-r(D) \lambda_{s+1}\right)$.

The first upper bound is established.

To prove the second inequality, one goes back over the previous inequalities replacing the Lyapunov exponent $\lambda_{s+1}$ by $\lambda_{s}$ in the bound of $\left\|\tilde{A}_{h_{p(u)}, h_{u}}\right\|$ (since $h_{u}>h_{p(u)}$ for $\left.u \in \mathfrak{i}_{2}(D)\right)$ and in the bound of $\left\|(\mathrm{I}-\Pi) A_{h_{\rho}-1}\left(\theta^{-\left(h_{\rho}-1\right)}\right)\right\|$. Thus, the second upper bound is based on the following inequalities:

$$
\begin{array}{r}
\|f(D ; h)(0)\| \leq C_{\epsilon}^{1+\ell_{\rho}} C_{\epsilon}^{\left(m_{\rho}\right)} \mathrm{e}^{-\lambda_{s}} \exp \left(h_{\rho}\left(\left(1-\ell_{\rho}\right) \lambda_{s}+\left(2+\ell_{\rho}\right) \epsilon\right)\right) \\
\prod_{i=1}^{r_{\rho}}\left\|f_{h_{\rho}}\left(D_{\rho_{i}} ; h_{\mid D_{\rho_{i}}}\right)(0)\right\|,
\end{array}
$$

where $\rho_{1}, \ldots, \rho_{r_{\rho}}$ denote the non-terminal sons of the root $\rho$ and where for every $u \in \mathfrak{i}(D)^{*}$,

$$
\begin{aligned}
\left\|f_{h_{p(u)}}\left(D_{u} ; h_{\mid D_{u}}\right)(0)\right\| & \leq C_{\epsilon}^{1+\ell_{u}} C_{\epsilon}^{\left(m_{u}\right)} \mathrm{e}^{-\lambda_{s}} \exp \left(h_{p(u)}\left(-\lambda_{s}+\epsilon\right)\right) \\
& \exp \left(h_{u}\left(\lambda_{s}\left(1-\ell_{u}\right)+\left(2+\ell_{u}\right) \epsilon\right)\right) \prod_{i=1}^{r}\left\|f_{h_{u}}\left(D_{u_{i}} ; h_{\mid D_{u_{i}}}\right)(0)\right\| .
\end{aligned}
$$

3.2.1. Case $\lambda_{s}>0$. - Let us begin with the case $\lambda_{s}>0$ for which the proof of Lemma 3.7 is straightforward. The second upper bound given by Lemma 3.9 is sufficient to prove that, for every diagram $D \in \bigcup_{i=2}^{k} \mathcal{T}_{i}$, the sequence $\left(\sum_{h \in H_{n}(D)}\|f(D ; h)(0)\|\right)_{n}$ converges $\mathbb{P}$-almost surely when $\lambda_{s}>0$ :

Lemma 3.10. - Assume that $\lambda_{s}>0$ and $0<\epsilon<\frac{1}{5} \lambda_{s}$. For every diagram $D \in \bigcup_{i=2}^{k} \mathcal{T}_{i}$, the following sum is finite:

$$
\sum_{h \in H(D)} \exp \left(\sum_{u \in \mathfrak{i}(D)} h_{u}\left(\lambda_{s}\left(1-m_{u}\right)+\epsilon\left(2+m_{u}\right)\right)\right) .
$$

Proof. - If $u \in \mathfrak{i}(D)$, then $\lambda_{s}\left(1-m_{u}\right)+\epsilon\left(2+m_{u}\right) \leq \lambda_{s}\left(\frac{7}{5}-\frac{4}{5} m_{u}\right)$ and $m_{u} \geq 2$. Thus, for all $u \in \mathfrak{i}(D), \lambda_{s}\left(1-m_{u}\right)+\epsilon\left(2+m_{u}\right) \leq-\frac{1}{5} \lambda_{s}$.

TOME $129-2001-\mathrm{N}^{\mathrm{O}} 3$ 
3.2.2. Case $\lambda_{s} \leq 0$ and $\lambda_{s+1}-k \lambda_{s}<0$. - Let us now assume that $\lambda_{s} \leq 0$ and $\lambda_{s+1}-k \lambda_{s}<0$. Using the first inequality given by Lemma 3.10, it remains to prove that there exists $\epsilon>0$ such that for all $D \in \mathcal{T}_{k}$ the sequence

$$
\left(\sum_{h \in H_{n}(D)} \exp \left(\sum_{u \in \mathfrak{i}_{1}(D)} h_{u} \alpha_{u}\right)\right)_{n}
$$

converges, where $\left(\alpha_{u}\right)_{u \in \mathfrak{i}(D)}$ is a family of reals depending on $\epsilon$, defined by (21).

The following lemma gives a simple criterion to check whether such a geometric sum converges:

Lemma 3.11. - Let $D=(X, E)$ be a diagram without cycle and let $\beta=\left(\beta_{u}\right)_{u \in X}$ be a family of reals indexed by $X$. For $n \in \mathbb{N}^{*}$, let $\mathcal{H}_{n}(D)$ denote the set of families, $h=\left(h_{u}\right)_{u \in X}$, of positive integers smaller than $n$, indexed by $X$ and satisfying $h_{u} \leq h_{v}$ for all $(u, v) \in E$. Let $\mathcal{P}(D)$ be the set of non-empty subsets of $X$, closed in $D$. For $J \in \mathcal{P}(D)$, let $Q(J, \beta)$ denote the set of subsets $K$ of $J$ such that $\sum_{v \in K} \beta_{v}=0$. There exists a constant $C(\beta, D)$ such that for all $n \in \mathbb{N}^{*}$ :

$$
\sum_{h \in \mathcal{H}_{n}(D)} \exp \left(\sum_{u \in X} \beta_{u} h_{u}\right) \leq C(\beta, D) \max \left(1, \max _{J \in \mathcal{P}(D)}\left(n^{|Q(J, \beta)|} \exp \left(n \sum_{v \in J} \beta_{v}\right)\right)\right)
$$

In particular, a sufficient condition for the sequence

$$
\left(\sum_{h \in \mathcal{H}_{n}(D)} \exp \left(\sum_{u \in X} \beta_{u} h_{u}\right)\right)_{n}
$$

to converge is

$$
\forall J \in \mathcal{P}(D), \quad \sum_{v \in J} \beta_{v}<0 .
$$

Proof. — The proof is by induction on the number of roots of the diagram $D$.

1) The result holds if $D$ has one node. Indeed,

$$
\sum_{h=1}^{n} \mathrm{e}^{h \beta}= \begin{cases}n & \text { if } \beta=0 \\ \frac{\mathrm{e}^{\beta}-\mathrm{e}^{(n+1) \beta}}{1-\mathrm{e}^{\beta}} & \text { if } \beta \neq 0\end{cases}
$$

Thus,

$$
\sum_{h=1}^{n} \mathrm{e}^{h \beta} \leq C(\beta, D) \max \left(1, n^{1_{\beta=0}} \mathrm{e}^{n \beta}\right)
$$

where $C(\beta, D)=\mathrm{e}^{\beta} /\left|1-\mathrm{e}^{\beta}\right| 1_{\beta \neq 0}+1_{\beta=0}$.

2) Let $k \in \mathbb{N}^{*}$. Assume that the result holds for all diagrams without cycle and with at most $k$ nodes. Consider a diagram $D=(X, E)$ without cycle and with $k+1$ nodes. As $D$ has no cycle and has a finite number of nodes, it has 
at least one terminal node. Let $u$ denote a terminal node of $D$ and $\mathfrak{p}(u)$ the set (eventually empty) of the predecessors of $u$ :

$$
\mathfrak{p}(u)=\{x \in X,(x, u) \in E\} .
$$

The subdiagram $\check{D}=(\check{X}, \check{E})$, obtained by removing from $D$ the node $u$ and every link $(x, u)$ for $x \in \mathfrak{p}(u)$, is a diagram with no cycle and with $k$ nodes. One may note that as $u$ is a terminal node, $\{u\} \in \mathcal{P}(D)$ and that if $J \in \mathcal{P}(\check{D})$, then $J \cup\{u\} \in \mathcal{P}(D)$.

Two cases will be distinguished depending on whether $\beta_{u}$ is equal to zero or not. Set

$$
S_{n}=\sum_{h \in \mathcal{H}_{n}(D)} \exp \left(\sum_{v \in X} h_{v} \beta_{v}\right) .
$$

Case $\beta_{u}=0$. - The sum is bounded by

$$
S_{n} \leq n \sum_{h \in \mathcal{H}_{n}(\check{D})} \exp \left(\sum_{v \in \check{X}} h_{v} \beta_{v}\right) .
$$

Applying the induction hypothesis to the diagram $\check{D}$ and the restriction of the family $\beta$ to $\check{X}$ gives:

$$
S_{n} \leq C\left(\beta_{\mid \check{X}}, \check{D}\right) \max \left(n, \max _{J \in \mathcal{P}(\check{D})} n^{|Q(J, \beta)|+1} \exp \left(n \sum_{v \in J} \beta_{v}\right)\right) .
$$

If $J \in \mathcal{P}(\check{D})$, then $J \cup\{u\} \in \mathcal{P}(D)$ and $|Q(J, \beta)|+1 \leq|Q(J \cup\{u\}, \beta)|$. Furthermore,

$$
n=n^{|Q(\{u\}, \beta)|} \exp \left(n \sum_{v \in\{u\}} \beta_{v}\right) .
$$

Therefore, the result still holds for the diagram $D$ and the family $\beta$ when $\beta_{u}=0$.

Case $\beta_{u} \neq 0$.

(a) Assume first that $u$ has no predecessor. Then

$$
S_{n} \leq\left(S_{n}^{(1)}+S_{n}^{(2)}\right) \frac{\mathrm{e}^{\beta_{u}}}{\left|1-\mathrm{e}^{\beta_{u}}\right|}
$$

where

$$
S_{n}^{(1)}=\sum_{h \in \mathcal{H}_{n}(\check{D})} \exp \left(\sum_{v \in \check{X}} h_{v} \beta_{v}\right) \text { and } S_{n}^{(2)}=\sum_{h \in \mathcal{H}_{n}(\check{D})} \exp \left(\sum_{v \in \check{X}} h_{v} \beta_{v}+n \beta_{u}\right) .
$$

By the induction hypothesis,

$$
S_{n}^{(1)} \leq C\left(\beta_{\mid \check{X}}, \check{D}\right) \max \left(1, \max _{J \in \mathcal{P}(\check{D})}\left(n^{|Q(J, \beta)|} \exp \left(n \sum_{v \in J} \beta_{v}\right)\right)\right)
$$

TOME $129-2001-\mathrm{N}^{\mathrm{O}} 3$ 
and

$$
S_{n}^{(2)} \leq C\left(\beta_{\mid \check{X}}, \check{D}\right) \max \left(\mathrm{e}^{n \beta_{u}}, \max _{J \in \mathcal{P}(\check{D})}\left(n^{|Q(J, \beta)|} \exp \left(n \sum_{v \in J} \beta_{v}+n \beta_{u}\right)\right)\right) .
$$

As $u$ has no predecessor, $\mathcal{P}(\check{D}) \subset \mathcal{P}(D)$. Hence,

$$
S_{n}^{(1)} \leq C\left(\beta_{\mid \check{X}}, \check{D}\right) \max \left(1, \max _{J \in \mathcal{P}(D)}\left(n^{|Q(J, \beta)|} \exp \left(n \sum_{v \in J} \beta_{v}\right)\right)\right) .
$$

If $J \in \mathcal{P}(\check{D})$ then $J \cup\{u\} \in \mathcal{P}(D)$ and $|Q(J, \beta)| \leq|Q(J \cup\{u\}, \beta)|$. Furthermore $\mathrm{e}^{n \beta_{u}}=n^{|Q(\{u\}, \beta)|} \exp \left(n \sum_{v \in\{u\}} \beta_{v}\right)$. Therefore,

$$
S_{n}^{(2)} \leq C\left(\beta_{\mid \check{X}}, \check{D}\right) \max \left(1, \max _{J \in \mathcal{P}(D)}\left(n^{|Q(J, \beta)|} \exp \left(n \sum_{v \in J} \beta_{v}\right)\right)\right)
$$

and the result still holds for the diagram $D$ if $u$ has no predecessor.

(b) Assume now that $u$ has at least a predecessor denoted by $x$. Then,

$$
S_{n} \leq\left(S_{n}^{(2)}+S_{n}^{(3)}\right) \frac{\max \left(1, \mathrm{e}^{\beta_{u}}\right)}{\left|1-\mathrm{e}^{\beta_{u}}\right|}
$$

where

$$
S_{n}^{(3)}=\sum_{h \in \mathcal{H}_{n}(\check{D})} \exp \left(\sum_{v \in \mathfrak{i}(\check{D})} h_{v} \beta_{v}\right) \exp \left(h_{x} \beta_{x}\right) .
$$

The proof of the bound for $S_{n}^{(2)}$ does not use the fact that $u$ has no predecessor, thus the bound for $S_{n}^{(2)}$ still holds.

To study $S_{n}^{(3)}$, one applies the induction hypothesis to the diagram $\check{D}$ and the family $\tilde{\beta}=\left(\tilde{\beta}_{u}\right)_{u \in \check{X}}$, where

$$
\tilde{\beta}_{v}= \begin{cases}\beta_{x}+\beta_{u} & \text { if } v=x \\ \beta_{v} & \text { if } v \in \check{D} \backslash\{x\} .\end{cases}
$$

One has

$$
S_{n}^{(3)} \leq C(\tilde{\beta}, \check{D}) \max \left(1, \max _{J \in P(\tilde{D})}\left(n^{|Q(J, \tilde{\beta})|} \exp \left(n \sum_{v \in J} \tilde{\beta}_{v}\right)\right)\right) .
$$

Let $J \in P(\check{D})$.

- If $x \notin J$, then $J \in \mathcal{P}(D)$ and

$$
n^{|Q(J, \tilde{\beta})|} \exp \left(n \sum_{v \in J} \tilde{\beta}_{v}\right)=n^{|Q(J, \beta)|} \exp \left(n \sum_{v \in J} \beta_{v}\right) .
$$

- If $x \in J$, then $\sum_{v \in J} \tilde{\beta}_{v}=\sum_{v \in J \cup\{u\}} \beta_{v}$ and $J \cup\{u\} \in \mathcal{P}(D)$. Furthermore, $|Q(J, \tilde{\beta})| \leq|Q(J \cup\{u\}, \beta)|$, since for $K \in Q(J, \tilde{\beta})$ :

$\triangleright$ if $x \notin K$, then $K \in Q(J \cup\{u\}, \beta)$;

$\triangleright$ if $x \in K$, then $K \cup\{u\} \in Q(J \cup\{u\}, \beta)$.

BULlETiN DE LA SOCIÉtÉ MATHÉMATIQUE DE FRANCE 
Therefore,

$$
n^{|Q(J, \tilde{\beta})|} \exp \left(n \sum_{v \in J} \tilde{\beta}_{v}\right) \leq n^{|Q(J \cup\{u\}, \beta)|} \exp \left(n \sum_{v \in J \cup\{u\}} \beta_{v}\right)
$$

and

$$
S_{n}^{(3)} \leq C\left(\beta_{\mid \check{X}}, \check{D}\right) \max \left(1, \max _{J \in \mathcal{P}(D)}\left(n^{|Q(J, \beta)|} \exp \left(n \sum_{v \in J} \beta_{v}\right)\right)\right) .
$$

The result is also true when $u$ has a predecessor. This closes the proof of Lemma 3.11.

Consider a diagram $D=\left(X, E_{1}, E_{2}, I_{1}, I_{2}\right) \in \mathcal{T}_{k}$ and fix a positive real $\epsilon$ smaller than $\left(k \lambda_{s}-\lambda_{s+1}\right) /(4 k)$. Let $\alpha=\left(\alpha_{u}\right)_{u \in \mathfrak{i}(D)}$ denote the family of reals defined by:

- $\alpha_{u}=\lambda_{s}\left(1-\ell_{u}-r_{u}^{(1)}\right)-r_{u}^{(2)} \lambda_{s+1}+\epsilon\left(2+m_{u}\right)$ for $u \in \mathfrak{i}_{1}(D)$;

- $\alpha_{u}=-\lambda_{s}\left(\ell_{u}+r_{u}^{(1)}\right)+\lambda_{s+1}\left(1-r_{u}^{(2)}\right)+\epsilon\left(2+m_{u}\right)$ for $u \in \mathfrak{i}_{2}(D) \cup\{\rho(D)\}$.

Lemmas 3.9 and 3.11 insure that $\left(\sum_{h \in H_{n}(D)}\|f(D ; h)(0)\|\right)_{n}$ converges if for all $J \in \mathcal{P}\left(\left(X, I_{1}\right)\right)$, the sum $\sum_{u \in J} \alpha_{u}$ is negative. To shorthand the notations, set

$$
\alpha(K):=\sum_{u \in \mathfrak{i}(K)} \alpha_{u}
$$

for any subset $K$ of $X$.

Consider a set $J \in \mathcal{P}\left(\left(X, I_{1}\right)\right)$. As the expression of $\alpha_{u}$ differs depending on whether $u \in \mathfrak{i}_{1}(D)$ or $u \in \mathfrak{i}_{2}(D) \cup\{\rho(D)\}$, it will be useful to isolate the nodes of $\mathfrak{i}_{1}(D)$. Let $\rho_{1}:=\rho(D)$ and let $\rho_{2}, \ldots, \rho_{q}$ denote the elements of $\mathfrak{i}_{1}(D)$. For $v \in X$, let $C(v)$ denote the subset of $X$ which consists of $v$ and its descendants in the diagram $\left(X, E_{2}\right)$. Finally, set $C^{i}:=C\left(\rho_{i}\right)$ for $i \in\{1, \ldots, q\}$. As $\left(X, E_{1} \cup E_{2}\right)$ is a tree, the sets $C^{1}, \ldots, C^{q}$ define a partition of $X$ into closed subsets in the diagram $\left(X, E_{2}\right)$. Thus, $J$ is the disjoint union of the sets $J \cap C^{x}$ for $x \in\{1, \ldots, q\}$. One has to prove that

$$
\alpha(J)=\sum_{x=1}^{q} \alpha\left(J \cap C^{x}\right) 1_{J \cap C^{x} \neq \varnothing}<0 .
$$

As $J$ is closed in $\left(X, I_{1}\right)$, a node in $\mathfrak{i}_{2}(D)$ whose predecessor belongs to $J$, is also in $J$. Thus, for every $x \in\{1, \ldots, q\}$, if $v \in J \cap C^{x}$ then $\mathfrak{i}(C(v)) \subset J \cap C^{x}$. This implies that if $J \cap C^{x} \neq \varnothing$, then there exist $s_{x} \geq 1$ interior nodes, denoted by $v_{1}^{x}, \ldots, v_{s_{x}}^{x}$, such that $J \cap C^{x}$ is equal to the disjoint union $\bigcup_{j=1}^{s_{x}} \mathfrak{i}\left(C\left(v_{j}^{x}\right)\right)$. Let us begin by computing a bound of $\alpha(C(v))$ for all $v \in \mathfrak{i}(D)$ :

$$
\begin{array}{r}
\alpha(C(v))=\lambda_{s+1}\left(|\mathfrak{i}(C(v))|-r^{(2)}(C(v))\right)-\lambda_{s}\left(\ell(C(v))+r^{(1)}(C(v))\right) \\
+\epsilon(m(C(v))+2|\mathfrak{i}(C(v))|) \text { if } v \in \mathfrak{i}_{2}(D) \cup\{\rho(D)\},
\end{array}
$$

TOME $129-2001-\mathrm{N}^{\mathrm{O}} 3$ 


$$
\begin{gathered}
\alpha(C(v))=\lambda_{s+1}\left(|\mathfrak{i}(C(v))|-1-r^{(2)}(C(v))\right)+\lambda_{s}\left(1-\ell(C(v))-r^{(1)}(C(v))\right) \\
+\epsilon(m(C(v))+2|\mathfrak{i}(C(v))|) \text { if } v \in \mathfrak{i}_{1}(D) .
\end{gathered}
$$

As $C(v)$ is closed in the diagram $\left(X, E_{2}\right)$, the number of its interior nodes satisfies $|\mathfrak{i}(C(v))|=1+r^{(2)}(C(v))$. Thus, if $v \in \mathfrak{i}_{2}(D) \cup\{\rho(D)\}$,

$$
\alpha(C(v))=\lambda_{s+1}-\lambda_{s}\left(\ell(C(v))+r^{(1)}(C(v))\right)+\epsilon(m(C(v))+2|\mathfrak{i}(C(v))|)
$$

and if $v \in \mathfrak{i}_{1}(D)$,

$$
\alpha(C(v))=\lambda_{s}\left(1-\ell(C(v))-r^{(1)}(C(v))\right)+\epsilon(m(C(v))+2|\mathfrak{i}(C(v))|) .
$$

As $\lambda_{s+1}<\lambda_{s}<0$, for all $v \in \mathfrak{i}(D)$,

$$
\alpha(C(v)) \leq \lambda_{s}\left(1-\ell(C(v))-r^{(1)}(C(v))\right)+\epsilon(m(C(v))+2|\mathfrak{i}(C(v))|) .
$$

It happens that $\ell(C(v))+r^{(1)}(C(v))>1$ for all $v \in \mathfrak{i}(D)$. Indeed, let

$$
D(v)=(X(v), E(v))
$$

denote the subdiagram of $\left(X, E_{1} \cup E_{2}\right)$ such that the set of nodes $X(v)$ is the union of $C(v)$ and of the sons of the elements of $C(v)$, and such that the set of links is

$$
E(v)=\left(E_{1} \cup E_{2}\right) \cap(X(v) \times X(v)) .
$$

The diagram $D(v)$ is a tree whose interior nodes have at least two sons and whose terminal nodes are either leaves of $\left(X, E_{1} \cup E_{2}\right)$, or elements of $\mathfrak{i}_{1}(D)$. Thus, $\ell(C(v))+r^{(1)}(C(v))$ is the number of leaves of $D(v)$. It is greater than the number of interior nodes of $D(v)$, that is greater than one.

It follows from inequality (23) that for all $x \in\{1, \ldots, q\}$,

$$
\begin{aligned}
& \alpha\left(J \cap C^{x}\right) \leq \lambda_{s}\left(1-\ell\left(J \cap C^{x}\right)-r^{(1)}\left(J \cap C^{x}\right)\right) \\
&+\epsilon\left(m\left(J \cap C^{x}\right)+2\left|\mathfrak{i}\left(J \cap C^{x}\right)\right|\right)
\end{aligned}
$$

and the upper bound is positive.

The upper bound in (24) can be improved when $J \cap C^{x}$ is a subset of $\mathfrak{i}_{2}(D) \cup\{\rho(D)\}:$ if $J \cap C^{x} \neq \varnothing$ and $J \cap C^{x} \subset \mathfrak{i}_{2}(D) \cup\{\rho(D)\}$, then

$$
\begin{aligned}
\alpha\left(J \cap C^{x}\right) \leq & \lambda_{s+1}-\lambda_{s}\left(\ell\left(J \cap C^{x}\right)+r^{(1)}\left(J \cap C^{x}\right)\right) \\
& +\epsilon\left(m\left(J \cap C^{x}\right)+2\left|\mathfrak{i}\left(J \cap C^{x}\right)\right|\right) \\
\leq & \lambda_{s+1}-\lambda_{s}\left(1-\ell\left(C^{x}\right)+r^{(1)}\left(C^{x}\right)\right)+\epsilon\left(m\left(C^{x}\right)+2\left|\mathfrak{i}\left(C^{x}\right)\right|\right) .
\end{aligned}
$$

As the diagram $\left(X, E_{1} \cup E_{2}\right)$ has $k$ leaves, for every $x \in\{1, \ldots, q\}$,

$$
r^{(1)}\left(C^{x}\right)+\ell\left(C^{x}\right) \leq k .
$$

Indeed, suppose to the contrary that there exists $i_{0} \in\{1, \ldots, q\}$ such that $r^{(1)}\left(C^{i_{0}}\right)+\ell\left(C^{i_{0}}\right) \geq k+1$. Then

$$
\ell(D)=\sum_{i=1}^{q}\left(\ell\left(C^{i}\right)+r^{(1)}\left(C^{i}\right)\right)-(q-1)
$$

BULLETIN DE LA SOCIÉtÉ MATHÉMATIQUE DE FRANCE 
is greater than or equal to $k+1+2(q-1)-(q-1)=k+q$, that is contradictory to the fact that $\left(X, E_{1} \cup E_{2}\right)$ has $k$ leaves.

On the other hand,

$m\left(C^{x}\right)+2\left|\mathfrak{i}\left(C^{x}\right)\right| \leq m(D)+2|\mathfrak{i}(D)|=1+3|\mathfrak{i}(D)|+\ell(D) \leq 4 \ell(D)-2=4 k-2$.

As $\epsilon$ is taken smaller than $\left(k \lambda_{s}-\lambda_{s+1}\right) /(4 k)$, the right term in (25) is negative.

The condition " $J \cap C^{x}$ is a subset of $\mathfrak{i}_{2}(D) \cup \rho(D)$ " is satisfied whether $x=1$, or $x \in\{2, \ldots, q\}$ and $J \cap C^{x}$ is strictly included in $\mathfrak{i}\left(C^{x}\right)$. Thus $\alpha(J)$ is bounded by

$$
\begin{aligned}
\alpha(J) \leq( & \left.\lambda_{s+1}-\lambda_{s}\left(\ell\left(C^{1}\right)+r^{(1)}\left(C^{1}\right)\right)+\epsilon\left(m\left(C^{1}\right)+2\left|\mathfrak{i}\left(C^{1}\right)\right|\right)\right) 1_{J \cap C^{1} \neq \varnothing} \\
& +\sum_{x=2}^{q}\left(\lambda_{s+1}-\lambda_{s}\left(1-\ell\left(C^{x}\right)-r^{(1)}\left(C^{x}\right)\right)\right. \\
& \left.\left.+\sum_{x=2}^{q}\left(\lambda_{s}\left(1-\ell\left(C^{x}\right)+2\left|\mathfrak{i}\left(C^{x}\right)\right|\right)\right) 1_{J \cap C^{x} \notin\left\{\varnothing, \mathfrak{i}\left(C^{x}\right)\right\}}-r^{(1)}\left(C^{x}\right)\right)+\epsilon\left(m\left(C^{x}\right)+2\left|\mathfrak{i}\left(C^{x}\right)\right|\right)\right) 1_{\mathfrak{i}\left(C^{x}\right) \subset J} .
\end{aligned}
$$

The following lemma describes the subsets $J \cap C^{x}$ :

Lemma 3.12. - A subset $J \in \mathcal{P}\left(\left(X, I_{1}\right)\right)$ satisfies at least one of these two assertions:

(i) $J \cap C^{1} \neq \varnothing$;

(ii) there exists an index $\ell \in\{2, \ldots, q\}$ such that $J \cap C^{\ell} \notin\left\{\varnothing, \mathfrak{i}\left(C^{\ell}\right)\right\}$.

Proof. - Assume that for every $\ell \in\{2, \ldots, q\}, C^{\ell} \cap J \neq \varnothing \Rightarrow \mathfrak{i}\left(C^{\ell}\right) \subset J$. One has to show that under this assumption, $J \cap C^{1} \neq \varnothing$. Let $x \in J$. As $\left(X, E_{1} \cup E_{2}\right)$ is a tree with root $\rho_{1} \in \mathfrak{i}\left(C^{1}\right)$, there exists a path $\left(x_{1}, \ldots, x_{r}\right)$ in $\left(X, E_{1} \cup E_{2}\right)$ going from $\rho_{1}$ to $x$. Thus, to prove that $C^{1} \cap J \neq \varnothing$, it suffices to establish the following property:

$(\star)$ If there exists a path in $\left(X, E_{1} \cup E_{2}\right)$ connecting a node in $\mathfrak{i}\left(C^{1}\right)$ with a node in $J$, then $C^{1} \cap J \neq \varnothing$.

This property can be proved by induction on the number of nodes lying in $\mathfrak{i}_{1}(D)$ in a path that connects a node of $\mathfrak{i}\left(C^{1}\right)$ with a node of $J$.

1) Let $\underline{x}=\left(x_{1}, \ldots, x_{r}\right)$ be a path in $\left(X, E_{1} \cup E_{2}\right)$ such that $x_{1} \in \mathfrak{i}\left(C^{1}\right)$, $x_{r} \in J$ and $x_{i} \in \mathfrak{i}_{2}(D) \cup\{\rho(D)\}$ for all $i \in\{1, \ldots, r\}$. Then the set $\mathfrak{i}\left(C^{1}\right)$ contains the path $\underline{x}$. Therefore, $C^{1} \cap J \neq \varnothing$.

$2)$ Let $j \in \mathbb{N}$. Assume that $(\star)$ holds for every path having $j$ nodes that belong to $\mathfrak{i}_{1}(D)$. Consider a path $\left(x_{1}, \ldots, x_{r}\right)$ in $\left(X, E_{1} \cup E_{2}\right)$ such that $x_{1} \in \mathfrak{i}\left(C^{1}\right)$, $x_{r} \in J$ and such that $j+1$ nodes belong to $\mathfrak{i}_{1}(D)$. Set

$$
t:=\max \left\{i \in\{1, \ldots, r\} \text { such that } x_{i} \in \mathfrak{i}_{1}(D)\right\} .
$$

TOME $129-2001-\mathrm{N}^{\mathrm{O}} 3$ 
If $x_{r} \in \mathfrak{i}\left(C^{1}\right)$, then $C^{1} \cap J \neq \varnothing$. Otherwise, there exists $\ell \in\{2, \ldots, q\}$ such that $x_{r} \in \mathfrak{i}\left(C^{\ell}\right)$. In this case, $C^{\ell} \cap J \neq \varnothing$ and the assumption implies that $\mathfrak{i}\left(C^{\ell}\right) \subset J$. By construction, $x_{t} \in \mathfrak{i}\left(C^{\ell}\right) \subset J$ and $x_{t} \in \mathfrak{i}_{1}(D)$. It follows that $\left(x_{t}, x_{t-1}\right) \in I_{2} \subset I_{1}$ and as $J$ is closed in $\left(X, I_{1}\right), x_{t-1} \in J$. Hence, the path $\left(x_{1}, \ldots, x_{t-1}\right)$ in $\left(X, E_{1} \cup E_{2}\right)$ relates a node $x_{1} \in \mathfrak{i}\left(C^{1}\right)$ to a node $x_{t-1} \in J$ and contains $j$ nodes that belong to $\mathfrak{i}_{1}(D)$. Then, the induction hypothesis implies that $C^{1} \cap J \neq \varnothing$.

Lemma 3.12 and the fact that the right term of (25) is negative imply that

$$
\begin{aligned}
\alpha(J) \leq \max _{1 \leq j \leq q}\{ & \lambda_{s+1}-\lambda_{s}\left(\ell\left(C^{j}\right)+r^{(1)}\left(C^{j}\right)\right)+\epsilon\left(m\left(C^{j}\right)+2\left|\mathfrak{i}\left(C^{j}\right)\right|\right) \\
& \left.+\sum_{\substack{x=2 \\
x \neq j}}^{q}\left(\lambda_{s}\left(1-\ell\left(C^{j}\right)-r^{(1)}\left(C^{j}\right)\right)+\epsilon\left(m\left(C^{j}\right)+2\left|\mathfrak{i}\left(C^{j}\right)\right|\right)\right)\right\} .
\end{aligned}
$$

As $\sum_{x=1}^{q}\left(r^{(1)}\left(C^{x}\right)+\ell\left(C^{x}\right)-1\right)+1=q-1+k-q+1=k$ and

$$
\sum_{x=1}^{q}\left(m\left(C^{x}\right)+2\left|\mathfrak{i}\left(C^{x}\right)\right|\right)=m(D)+2|\mathfrak{i}(D)| \leq 4 k-2,
$$

it turns out that $\alpha(J) \leq \lambda_{s+1}-k \lambda_{s}+(4 k-2) \epsilon<0$.

According to Lemma 3.11, the following lemma is established:

LEMma 3.13. - Assume that $\lambda_{s+1}-k \lambda_{s}<0$ and that $\lambda_{s}<0$. Fix $\epsilon>0$ smaller than $\left(k \lambda_{s}-\lambda_{s+1}\right) /(4 k)$ and set

$$
\alpha_{u}= \begin{cases}-\lambda_{s}\left(\ell_{u}+r_{u}^{(1)}\right)+\left(1-r_{u}^{(2)}\right) \lambda_{s+1}+\epsilon\left(2+m_{u}\right) & \text { if } u \in \mathfrak{i}_{2}(D) \cup\{\rho(D)\}, \\ \lambda_{s}\left(1-\ell_{u}-r_{u}^{(1)}\right)-r_{u}^{(2)} \lambda_{s+1}+\epsilon\left(2+m_{u}\right) & \text { if } u \in \mathfrak{i}_{1}(D) .\end{cases}
$$

For every $D \in \mathcal{T}_{k}$, the following sum is finite:

$$
\left(\sum_{h \in H(D)} \exp \left(\sum_{u \in \mathfrak{i}_{1}(D)} h_{u} \alpha_{u}\right)\right) \text {. }
$$

3.3. Convergence of the derivatives of $\boldsymbol{\Gamma}_{\boldsymbol{n}}$ at zero. - Let us assume that for $j \in\{1, \ldots, k\}, \log ^{+}\left(\left\|B^{(j)}\right\|\right) \in L^{1}(\Omega, \mathcal{A}, \mathbb{P})$ and that

$$
D \xi(0)\left(\mathbb{R}^{d}\right) \cap \bigoplus_{i=s+1}^{r} E_{i}=\{0\}
$$

$\mathbb{P}$-almost surely. The aim of this subsection is to prove that, if $\lambda_{s+1}-k \lambda_{s}<0$, then $\left(D^{k} \Gamma_{n}(0)-D^{k} \Upsilon_{n}(0)\right)_{n}$ converges to zero in probability.

Recall that $\Gamma_{n}$ is the orthogonal normalization of $\psi_{n+1}$ in the Case 2, i.e. when $\phi_{n+1}$ is the restriction of $\phi_{n}\left(\theta^{-n}\right) \circ \xi\left(\theta^{-n}\right)$ to a neighbourhood of 0 in $\mathbb{R}^{d}$, whereas $\Upsilon_{n}$ is the orthogonal normalization of $\psi_{n}$ in the Case 1, i.e. when $\phi_{n}$ is the restriction of $\phi_{n}\left(\theta^{-n}\right)$ to a neighbourhood of 0 in $E^{s}\left(\theta^{-n}\right)$. As the 
derivatives of the two applications, $\Upsilon_{n}$ and $\Gamma_{n}$, will be used simultaneously, the number 1 or 2 will be added in the notations of the applications $F_{n}(D)$, $f_{n}(D ; h), a_{i, j, n}$, etc. whether they are defined in the Case 1 or 2 .

By Proposition 3.1, there exists a family of reals $\{c(D)\}_{D \in \mathcal{T}_{k}}$, such that for all $n \in \mathbb{N}^{*}$,

$$
D^{k} \Upsilon_{n}(0)=\sum_{D \in \mathcal{T}_{k}} c(D) F_{n}(1 ; D)(0), \quad D^{k} \Gamma_{n}(0)=\sum_{D \in \mathcal{T}_{k}} c(D) F_{n+1}(2 ; D)(0) .
$$

Each term $F_{n+1}(2 ; D)(0)$ can be split into the sum of two terms:

$$
\begin{aligned}
& F_{n+1}^{1}(2 ; D)(0):=\sum_{h \in H_{n}(D)} f_{n+1}(2 ; D ; h)(0), \\
& F_{n+1}^{2}(2 ; D)(0):=\sum_{h \in H_{n+1}(D) \backslash H_{n}(D)} f_{n+1}(2 ; D ; h)(0) .
\end{aligned}
$$

The first term, $F_{n+1}^{1}(2 ; D)(0)$ does not depend on the derivatives of $\xi$ of orders greater than 1 . For every $D \in \mathcal{T}_{k}$, the convergence to 0 of the sequences $\left(F_{n+1}^{1}(2 ; D)(0)-F_{n}(1 ; D)(0)\right)_{n}$ and $\left(F_{n+1}^{2}(2 ; D)(0)\right)_{n}$ will be successively established.

3.3.1. Convergence of $\left(F_{n+1}^{1}(2 ; D)(0)-F_{n}(1 ; D)(0)\right)_{n}$ to 0 . - For $h \in H_{n}(D)$, the expression of $f_{n+1}(2 ; D ; h)(0)$ can be deduced from the expression of $f_{n}(1 ; D ; h)(0)$ by replacing each projector $\Pi$ by a projector $\Pi_{n}$. Thus, the splitting of

- each vector $\tilde{a}_{i, j, n}(2)(0)$ into the sum of the vector

$$
\tilde{a}_{i, j, n}(2)(0)-\tilde{a}_{i, j, n}(1)(0)=A_{-i}\left(\Pi-\Pi_{n}\right) A_{j}\left(\theta^{-j}\right)
$$

and the vector $\tilde{a}_{i, j, n}(1)(0)=A_{-i}(\mathrm{I}-\Pi) A_{j}\left(\theta^{-j}\right)$,

- each projector $p_{n}(2)(0)=\Pi_{n}$ into the sum of $p_{n}(2)-p_{n}(1)=\Pi_{n}-\Pi$ and $p_{n}(1)=\Pi$,

in the expression of $F_{n+1}(2 ; D)(0)$, gives an expression of $F_{n+1}^{1}(D ; 2)(0)-$ $F_{n}(D ; 1)(0)$ which is a combination (independent of $n$ ) of terms that contain at least one operator $\Pi_{n}-\Pi$. To describe these terms set:

$$
\begin{array}{rlrl}
\bar{a}_{i, j, n}(1) & :=A_{-i}\left(\Pi-\Pi_{n}\right) A_{j}\left(\theta^{-j}\right), \bar{a}_{i, j}(0) & :=A_{-i}(\mathrm{I}-\Pi) A_{j}\left(\theta^{-j}\right), \\
\bar{\kappa}_{n}(1) & :=\Pi_{n}-\Pi, & \bar{\kappa}_{n}(0) & :=\mathrm{I}-\Pi, \\
\bar{e}_{n}(1) & :=\Pi_{n}-\Pi, & \bar{e}_{n}(0) & :=\Pi .
\end{array}
$$

A diagram $D \in \mathcal{T}_{k}$ is now endowed with a family of heights $h \in H_{n}(D)$ and with two families of integers, denoted by

$$
\chi=\left(\chi_{u}\right)_{u \in \mathfrak{i}(D)} \text { and } \tau=\left(\tau_{u}\right)_{u \in \mathfrak{i}(D)},
$$

such that for all $u \in \mathfrak{i}(D), \chi_{u} \in\{0,1\}$ and $\tau_{u} \in\left\{0, \ldots, \ell_{u}\right\}$.

TOME $129-2001-\mathrm{N}^{\mathrm{O}} 3$ 
It remains to define the map associated with such a labelled diagram. First, for $u \in \mathfrak{i}_{1}(D)$ set

$$
\begin{aligned}
& g_{n, h_{p(u)}}\left(D_{u} ; h_{\mid D_{u}} ; \chi_{\mid D_{u}} ; \tau_{\mid D_{u}}\right):=A_{-h_{p(u)}} \bar{e}_{n}\left(\chi_{u}\right) A_{h_{u}-1}\left(\theta^{-\left(h_{u}-1\right)}\right) \\
& B^{\left(m_{u}\right)}\left(\theta^{-h_{u}}\right)\left(g_{n, h_{u}}\left(D_{u_{1}} ; h_{\mid D_{u_{1}}} ; \chi_{\mid D_{u_{1}}} ; \tau_{\mid D_{u_{1}}}\right), \ldots,\right. \\
& g_{n, h_{u}}\left(D_{u_{r_{u}}} ; h_{\mid D_{u_{r_{u}}}} ; \chi_{\mid D_{u_{r_{u}}}} ; \tau_{\mid D_{u_{r_{u}}}}\right) \text {, } \\
& \left.\left(A_{-h_{u}}\left(\Pi_{n}-\Pi\right)\right)^{\otimes \tau_{u}},\left(A_{-h_{u}} \Pi\right)^{\otimes\left(\ell_{u}-\tau_{u}\right)}\right)
\end{aligned}
$$

and for $u \in \mathfrak{i}_{2}(D)$

$$
\begin{aligned}
& g_{n, h_{p(u)}}\left(D_{u} ; h_{\mid D_{u}} ; \chi_{\mid D_{u}} ; \tau_{\mid D_{u}}\right):=\bar{a}_{h_{p(u)}, h_{u}-1, n}\left(\chi_{u}\right) \\
& B^{\left(m_{u}\right)}\left(\theta^{-h_{u}}\right)\left(g_{n, h_{u}}\left(D_{u_{1}} ; h_{\mid D_{u_{1}}} ; \chi_{\mid D_{u_{1}}} ; \tau_{\mid D_{u_{1}}}\right), \ldots,\right. \\
& g_{n, h_{u}}\left(D_{u_{r_{u}}} ; h_{\mid D_{u_{r_{u}}}} ; \chi_{\mid D_{u_{r_{u}}}} ; \tau_{\mid D_{u_{r_{u}}}}\right) \text {, } \\
& \left.\left(A_{-h_{u}}\left(\Pi_{n}-\Pi\right)\right)^{\otimes \tau_{u}},\left(A_{-h_{u}} \Pi\right)^{\otimes\left(\ell_{u}-\tau_{u}\right)}\right),
\end{aligned}
$$

where $u_{1}, \ldots, u_{r_{u}}$ designate the non-terminal sons of the node $u$.

The multilinear application $g_{n}(D ; h ; \chi ; \tau)$, associated with $(D ; h ; \chi ; \tau)$ is defined by

$$
\begin{aligned}
& g_{n}(D ; h ; \chi ; \tau)= \bar{\kappa}_{n}\left(\chi_{\rho}\right) A_{h_{\rho}-1}\left(\theta^{-\left(h_{\rho}-1\right)}\right) \\
& B^{\left(m_{\rho}\right)}\left(\theta^{-h_{\rho}}\right)\left(g_{n, h_{\rho}}\left(D_{\rho_{1}} ; h_{\mid D_{\rho_{1}}} ; \chi_{\mid D_{\rho_{1}}} ; \tau_{\mid D_{\rho_{1}}}\right), \ldots,\right. \\
& g_{n, h_{\rho}}\left(D_{\rho_{r_{\rho}}} ; h_{\mid D_{\rho_{r_{\rho}}}} ; \chi_{\mid D_{\rho_{r_{\rho}}}} ; \tau_{\mid D_{\rho_{r_{\rho}}}}\right) \\
&\left.\quad\left(A_{-h_{\rho}}\left(\Pi_{n}-\Pi\right)\right)^{\otimes \tau_{\rho}},\left(A_{-h_{\rho}} \Pi\right)^{\otimes\left(\ell_{\rho}-\tau_{\rho}\right)}\right),
\end{aligned}
$$

where $\rho_{1}, \ldots, \rho_{r_{\rho}}$ designate the non-terminal sons of the root $\rho$ of $D$.

With these notations, $F_{n+1}^{1}(2 ; D)(0)-F_{n}(1 ; D)(0)$ can be expressed as

$$
F_{n+1}^{1}(2 ; D)-F_{n}(1 ; D)(0)=\sum_{(\chi, \tau) \in \mathcal{Q}(D)} \beta(\chi, \tau) \sum_{h \in H_{n}(D)} g_{n}(D ; h ; \chi ; \tau)
$$

where

- $(\chi, \tau) \in \mathcal{Q}(D)$ if and only if $\chi=\left(\chi_{u}\right)_{u \in \mathfrak{i}(D)}$ and $\tau=\left(\tau_{u}\right)_{u \in \mathfrak{i}(D)}$ are two families of integers indexed by the interior nodes of $D$, satisfying the following properties: for all $u \in \mathfrak{i}(D), \chi_{u} \in\{0,1\}, \tau_{u} \in\left\{0, \ldots, \ell_{u}\right\}$ and $\sum_{u \in \mathfrak{i}(D)}\left(\chi_{u}+\tau_{u}\right) \geq 1$;

- $\beta(\chi, \tau)=\prod_{u \in \mathfrak{i}(D)} C_{\ell_{u}}^{\tau_{u}}$ for $(\chi, \tau) \in \mathcal{Q}(D)$.

The following lemma gives two upper bounds of the norm of the applications $g_{n}(D ; h ; \chi ; \tau)$ :

Lemma 3.14. - Let $D=\left(X, E_{1}, E_{2}, I_{1}, I_{2}\right) \in \bigcup_{i=1}^{k} \mathcal{T}_{i}$ and $(\chi, \tau) \in \mathcal{Q}(D)$. 
1) For all $0<\epsilon<\lambda_{s}-\lambda_{s+1}$, there exists a sequence of random variables $\left(C_{n, \epsilon}\right)_{n}$, converging in probability to 0 , such that for all $n \in \mathbb{N}$ and all $h \in H_{n}(D)$ :

$$
\left\|g_{n}(D ; h ; \chi ; \tau)\right\| \leq C_{n, \epsilon} \exp \left(\sum_{u \in \mathfrak{i}(D)} \beta_{u} h_{u}\right)
$$

where

$$
\beta_{u}= \begin{cases}\lambda_{s+1}\left(1-r_{u}^{(2)}\right)-\lambda_{s}\left(\ell_{u}+r_{u}^{(1)}\right)+\epsilon\left(3+2 m_{u}\right) & \text { if } u \in \mathfrak{i}_{1}(D), \\ -\lambda_{s+1} r_{u}^{(2)}+\lambda_{s}\left(1-\ell_{u}-r_{u}^{(1)}\right)+\epsilon\left(3+2 m_{u}\right) & \text { if } u \in \mathfrak{i}_{2}(D) \cup\{\rho(D)\} .\end{cases}
$$

2) For all $0<\epsilon<\lambda_{s}-\lambda_{s+1}$, there exists a sequence of random variables $\left(C_{n, \epsilon}\right)_{n}$, converging in probability to 0 , such that for all $n \in \mathbb{N}$ and all $h \in H_{n}(D):$ :

$$
\left\|g_{n}(D ; h ; \chi ; \tau)\right\| \leq C_{n, \epsilon} \exp \left(\sum_{u \in \mathfrak{i}(D)} \tilde{\beta}_{u} h_{u}\right)
$$

where $\tilde{\beta}_{u}=\lambda_{s}\left(1-m_{u}\right)+\epsilon\left(3+2 m_{u}\right)$ for all $u \in \mathfrak{i}(D)$.

Proof. - Set $\chi(D)=\sum_{u \in \mathfrak{i}(D)} \chi_{u}$ and $\tau(D)=\sum_{u \in \mathfrak{i}(D)} \tau_{u}$.

In addition to the usual bounds:

$$
\begin{aligned}
& \forall j \in\{1, \ldots, n\}, m \in\{2, \ldots, k\}, \quad\left\|B^{(m)}\left(\theta^{-j}\right)\right\| \leq C_{\epsilon}^{(m)} \mathrm{e}^{j \epsilon}, \\
& \forall j, 1 \leq j \leq n, \quad\left\|\bar{\kappa}_{n}(0) A_{j}\left(\theta^{-j}\right)\right\|=\left\|(\mathrm{I}-\Pi) A_{j}\left(\theta^{-j}\right)\right\| \leq C_{\epsilon} \mathrm{e}^{j\left(\lambda_{s+1}+\epsilon\right)}, \\
& \forall j, 1 \leq j \leq n, \quad\left\|A_{-j} \bar{e}_{n}(0)\right\|=\left\|A_{-j} \Pi\right\| \leq C_{\epsilon} \mathrm{e}^{j\left(-\lambda_{s}+\epsilon\right)}, \\
& \forall i, j, 0<i \leq j \leq n, \\
& \quad\left\|\bar{a}_{i, j, n}(0)\right\|=\left\|A_{-i}(\mathrm{I}-\Pi) A_{j}\left(\theta^{-j}\right)\right\| \leq C_{\epsilon} \mathrm{e}^{(j-i) \lambda_{s+1}+(j+i) \epsilon},
\end{aligned}
$$

$\forall i, j, 0<j \leq i \leq n$,

$$
\left\|A_{-i} \bar{e}_{n}(0) A_{j}\left(\theta^{-j}\right)\right\|=\left\|A_{-i} \Pi A_{j}\left(\theta^{-j}\right)\right\| \leq C_{\epsilon} \mathrm{e}^{(j-i) \lambda_{s}+(i+j) \epsilon},
$$

(where $C_{\epsilon}$ and $C_{\epsilon}^{(m)}$ for $m \in\{2, \ldots, k\}$ denote $\mathbb{P}$-almost surely finite random variables) the proof is based on the bound given by Proposition 2.1. Namely, for every $\epsilon>0$ there exists a sequence of random variables $\left(C_{\epsilon, n}\right)_{n}$ converging to zero in probability such that for all $n \in \mathbb{N}$, and $\ell, k \in\{0, \ldots, n\}$

$$
\left\|A_{-\ell}\left(\Pi_{n}-\Pi\right) A_{k}\left(\theta^{-k}\right)\right\| \leq C_{\epsilon, n} \mathrm{e}^{n\left(\lambda_{s+1}-\lambda_{s}+\epsilon\right)+k\left(\lambda_{s}+\epsilon\right)+\ell\left(-\lambda_{s+1}+\epsilon\right)} .
$$

Fix $0<\epsilon<\lambda_{s}-\lambda_{s+1}, n \in \mathbb{N}$ and $h \in H_{n}(D)$. Let us begin by establishing the following bound for the norm of the application $g_{n, h_{p(u)}}\left(D_{u} ; h_{\mid D_{u}} ; \chi_{\mid D_{u}} ; \tau_{\mid D_{u}}\right)$ for all $u \in \mathfrak{i}(D)^{*}$ : 
If $u \in \mathfrak{i}_{1}(D)$, then

$$
\begin{aligned}
& \left\|g_{n, h_{p(u)}}\left(D_{u} ; h_{\mid D_{u}} ; \chi_{\mid D_{u}} ; \tau_{\mid D_{u}}\right)\right\| \\
& \leq C_{\epsilon, n}^{\chi\left(D_{u}\right)+\tau\left(D_{u}\right)} C_{\epsilon}\left(D_{u}\right) \exp \left(h_{p(u)}\left(-\lambda_{s}+2 \epsilon\right)\right) \\
& \quad \exp \left(h_{u}\left(-\lambda_{s+1} r_{u}^{(2)}+\lambda_{s}\left(1-m_{u}+r_{u}^{(2)}\right)+\epsilon\left(3+2 m_{u}\right)\right)\right) .
\end{aligned}
$$

If $u \in \mathfrak{i}_{2}(D)$, then

$$
\begin{aligned}
& \left\|g_{n, h_{p(u)}}\left(D_{u} ; h_{\mid D_{u}} ; \chi_{\mid D_{u}} ; \tau_{\mid D_{u}}\right)\right\| \\
& \leq C_{\epsilon, n}^{\chi\left(D_{u}\right)+\tau\left(D_{u}\right)} C_{\epsilon}\left(D_{u}\right) \exp \left(h_{p(u)}\left(-\lambda_{s+1}+2 \epsilon\right)\right) \\
& \quad \exp \left(h_{u}\left(\lambda_{s+1}\left(1-r_{u}^{(2)}\right)-\lambda_{s}\left(m_{u}-r_{u}^{(2)}\right)+\epsilon\left(3+2 m_{u}\right)\right)\right) .
\end{aligned}
$$

where $C_{\epsilon}\left(D_{u}\right)$ is a $\mathbb{P}$-almost surely finite random variable.

Let $u$ be a node in $\mathfrak{i}_{1}(D)$,

$$
\begin{aligned}
& \left\|g_{n, h_{p(u)}}\left(D_{u} ; h_{\mid D_{u}} ; \chi_{\mid D_{u}} ; \tau_{\mid D_{u}}\right)\right\| \\
& \leq\left\|A_{-h_{p(u)}}\left(\Pi_{n}-\Pi\right) A_{h_{u}-1}\left(\theta^{-\left(h_{u}-1\right)}\right)\right\|^{\chi_{u}} \\
& \left\|A_{-h_{p(u)}} \Pi A_{h_{u}-1}\left(\theta^{-\left(h_{u}-1\right)}\right)\right\|^{1-\chi_{u}}\left\|B^{\left(m_{u}\right)}\left(\theta^{-h_{u}}\right)\right\| \cdot\left\|A_{-h_{u}}\left(\Pi_{n}-\Pi\right)\right\|^{\tau_{u}} \\
& \quad\left\|A_{-h_{u}} \Pi\right\|^{\ell_{u}-\tau_{u}} \prod_{i=1}^{r_{u}}\left\|g_{n, h_{u}}\left(D_{u_{i}} ; h_{\mid D_{u_{i}}} ; \chi_{\mid D_{u_{i}}} ; \tau_{\mid D_{u_{i}}}\right)\right\|
\end{aligned}
$$

where $u_{1}, \ldots, u_{r_{u}}$ designate the non-terminal sons of the node $u$.

It follows that:

$$
\begin{aligned}
& \left\|g_{n, h_{p(u)}}\left(D_{u} ; h_{\mid D_{u}} ; \chi_{\mid D_{u}} ; \tau_{\mid D_{u}}\right)\right\| \\
& \leq C_{\epsilon, n}^{\chi_{u}+\tau_{u}} C_{\epsilon}^{\left(m_{u}\right)} C_{\epsilon}^{1-\chi_{u}+\ell_{u}-\tau_{u}} \mathrm{e}^{-\lambda_{s}} \\
& \exp \left(\left(\chi_{u}+\tau_{u}\right) n\left(\lambda_{s+1}-\lambda_{s}+\epsilon\right)\right) \exp \left(h_{p(u)}\left(-\lambda_{s+1} \chi_{u}-\left(1-\chi_{u}\right) \lambda_{s}+\epsilon\right)\right) \\
& \exp \left(h_{u}\left(\lambda_{s}\left(1-\tau_{u}-\ell_{u}\right)-\lambda_{s+1} \tau_{u}+\epsilon\left(2+\ell_{u}\right)\right)\right) \\
& \prod_{i=1}^{r_{u}}\left\|g_{n, h_{u}}\left(D_{u_{i}} ; h_{\mid D_{u_{i}}} ; \chi_{\mid D_{u_{i}}} ; \tau_{\mid D_{u_{i}}}\right)\right\| .
\end{aligned}
$$

As $\lambda_{s+1}-\lambda_{s}+\epsilon<0$, and as the height of a node is lesser or equal to $n$,

$$
\begin{aligned}
& n\left(\lambda_{s+1}-\lambda_{s}+\epsilon\right) \chi_{u} \leq h_{p(u)}\left(\lambda_{s+1}-\lambda_{s}+\epsilon\right) \chi_{u} \\
& n\left(\lambda_{s+1}-\lambda_{s}+\epsilon\right) \tau_{u} \leq h_{u}\left(\lambda_{s+1}-\lambda_{s}+\epsilon\right) \tau_{u} .
\end{aligned}
$$

BULLETIN DE LA SOCIÉTÉ MATHÉMATIQUE DE FRANCE 
Therefore,

(28) $\left\|g_{n, h_{p(u)}}\left(D_{u} ; h_{\mid D_{u}} ; \chi_{\mid D_{u}} ; \tau_{\mid D_{u}}\right)\right\|$

$$
\begin{gathered}
\leq C_{\epsilon, n}^{\chi_{u}+\tau_{u}} C_{\epsilon}^{\left(m_{u}\right)} C_{\epsilon}^{1-\chi_{u}+\ell_{u}-\tau_{u}} \mathrm{e}^{-\lambda_{s}} \\
\prod_{i=1}^{r_{u}}\left\|g_{n, h_{u}}\left(D_{u_{i}} ; h_{\mid D_{u_{i}}} ; \chi_{\mid D_{u_{i}}} ; \tau_{\mid D_{u_{i}}}\right)\right\| \exp \left(h_{p(u)}\left(-\lambda_{s}+2 \epsilon\right)\right) \\
\quad \exp \left(h_{u}\left(\lambda_{s}\left(1-\ell_{u}\right) \tau_{u}+\epsilon\left(3+2 \ell_{u}\right)\right)\right) .
\end{gathered}
$$

Let $u$ be a node in $\mathfrak{i}_{2}(D)$,

$$
\begin{aligned}
& \left\|g_{n, h_{p(u)}}\left(D_{u} ; h_{\mid D_{u}} ; \chi_{\mid D_{u}} ; \tau_{\mid D_{u}}\right)\right\| \\
& \leq\left\|A_{-h_{p(u)}}\left(\Pi_{n}-\Pi\right) A_{h_{u}-1}\left(\theta^{-\left(h_{u}-1\right)}\right)\right\| \chi_{u} \\
& \left\|\tilde{A}_{-h_{p(u)}, h_{u}-1}\right\|^{1-\chi_{u}}\left\|B^{\left(m_{u}\right)}\left(\theta^{-h_{u}}\right)\right\| \cdot\left\|A_{-h_{u}}\left(\Pi_{n}-\Pi\right)\right\|^{\tau_{u}}\left\|A_{-h_{u}} \Pi\right\|^{\ell_{u}-\tau_{u}} \\
& \prod_{i=1}^{r_{u}}\left\|g_{n, h_{u}}\left(D_{u_{i}} ; h_{\mid D_{u_{i}}} ; \chi_{\mid D_{u_{i}}} ; \tau_{\mid D_{u_{i}}}\right)\right\| .
\end{aligned}
$$

Thus,

$$
\begin{aligned}
& \left\|g_{n, h_{p(u)}}\left(D_{u} ; h_{\mid D_{u}} ; \chi_{\mid D_{u}} ; \tau_{\mid D_{u}}\right)\right\| \\
& \leq C_{\epsilon, n}^{\chi_{u}+\tau_{u}} C_{\epsilon}^{\left(m_{u}\right)} C_{\epsilon}^{1-\chi_{u}+\ell_{u}-\tau_{u}} \\
& \quad \exp \left(-\chi_{u} \lambda_{s}-\left(1-\chi_{u}\right) \lambda_{s+1}+\left(\chi_{u}+\tau_{u}\right) n\left(\lambda_{s+1}-\lambda_{s}+\epsilon\right)\right) \\
& \quad \prod_{i=1}^{r_{u}}\left\|g_{n, h_{u}}\left(D_{u_{i}} ; h_{\mid D_{u_{i}}} ; \chi_{\mid D_{u_{i}}} ; \tau_{\mid D_{u_{i}}}\right)\right\| \exp \left(h_{p(u)}\left(-\lambda_{s+1}+\epsilon\right)\right) \\
& \quad \exp \left(h_{u}\left(\lambda_{s}\left(\chi_{u}+\tau_{u}-\ell_{u}\right)+\lambda_{s+1}\left(1-\chi_{u}-\tau_{u}\right)+\epsilon\left(2+\ell_{u}\right)\right)\right) .
\end{aligned}
$$

Replacing $n\left(\lambda_{s+1}-\lambda_{s}+\epsilon\right)$ by $h_{u}\left(\lambda_{s+1}-\lambda_{s}+\epsilon\right)$ gives:

$(29)\left\|g_{n, h_{p(u)}}\left(D_{u} ; h_{\mid D_{u}} ; \chi_{\mid D_{u}} ; \tau_{\mid D_{u}}\right)\right\|$

$$
\begin{aligned}
& \leq C_{\epsilon, n}^{\chi_{u}+\tau_{u}} C_{\epsilon}^{\left(m_{u}\right)} C_{\epsilon}^{1-\chi_{u}+\ell_{u}-\tau_{u}} \\
& \quad \exp \left(-\chi_{u} \lambda_{s}-\left(1-\chi_{u}\right) \lambda_{s+1}\right) \prod_{i=1}^{r_{u}}\left\|g_{n, h_{u}}\left(D_{u_{i}} ; h_{\mid D_{u_{i}}} ; \chi_{\mid D_{u_{i}}} ; \tau_{\mid D_{u_{i}}}\right)\right\| \\
& \quad \exp \left(h_{p(u)}\left(-\lambda_{s+1}+2 \epsilon\right)\right) \exp \left(h_{u}\left(-\lambda_{s} \ell_{u}+\lambda_{s+1}+\epsilon\left(3+2 \ell_{u}\right)\right)\right) .
\end{aligned}
$$

The bound of $\left\|g_{n, h_{p(u)}}\left(D_{u} ; h_{\mid D_{u}} ; \chi_{\mid D_{u}} ; \tau_{\mid D_{u}}\right)\right\|$ can be established by induction on the number of non-terminal descendants of $u$, with the help of inequalities (28) and (29).

Let $\rho$ denote the root of $D$ and $r_{1}, \ldots, r_{\rho_{r}}$ the non-terminal sons of $\rho$. To establish the first bound for $g$, it remains to consider the part of $g$ depending

TOME $129-2001-\mathrm{N}^{\mathrm{O}} 3$ 
on the height of the root:

$$
\begin{aligned}
& \left\|g_{n}(D ; h ; \chi ; \tau)\right\| \\
& \leq\left\|\left(\Pi_{n}-\Pi\right) A_{h_{\rho}-1}\left(\theta^{-\left(h_{\rho}-1\right)}\right)\right\|^{\chi_{\rho}}\left\|(\mathrm{I}-\Pi) A_{h_{\rho}-1}\left(\theta^{-\left(h_{\rho}-1\right)}\right)\right\|^{1-\chi_{\rho}} \\
& \left\|B^{\left(m_{\rho}\right)}\left(\theta^{-h_{\rho}}\right)\right\| \cdot\left\|A_{-h_{\rho}}\left(\Pi_{n}-\Pi\right)\right\|^{\tau_{\rho}}\left\|A_{-h_{\rho}} \Pi\right\|^{\ell_{\rho}-\tau_{\rho}} \\
& \quad \prod_{i=1}^{r_{\rho}}\left\|g_{n, h_{\rho}}\left(D_{\rho_{i}} ; h_{\mid D_{\rho_{i}}} ; \chi_{\mid D_{\rho_{i}}} ; \tau_{\mid D_{\rho_{i}}}\right)\right\| \\
& \leq C_{\epsilon, n}^{\chi_{\rho}+\tau_{\rho}} C_{\epsilon}^{\left(m_{\rho}\right)} C_{\epsilon}^{1-\chi_{\rho}+\ell_{\rho}-\tau_{\rho}} \mathrm{e}^{-\chi_{\rho} \lambda_{s}-\left(1-\chi_{\rho}\right) \lambda_{s+1}} \\
& \prod_{i=1}^{r_{\rho}}\left\|g_{n, h_{\rho}}\left(D_{\rho_{i}} ; h_{\mid D_{\rho_{i}}} ; \chi_{\mid D_{\rho_{i}}} ; \tau_{\mid D_{\rho_{i}}}\right)\right\| \mathrm{e}^{\left(\chi_{\rho}+\tau_{\rho}\right) n\left(\lambda_{s+1}-\lambda_{s}+\epsilon\right)} \\
& \quad \exp \left(h_{\rho}\left(\lambda_{s}\left(\chi_{\rho}+\tau_{\rho}-\ell_{\rho}\right)+\lambda_{s+1}\left(1-\chi_{\rho}-\tau_{\rho}\right)+\epsilon\left(2+\ell_{\rho}\right)\right)\right)
\end{aligned}
$$

Therefore,

$$
\begin{aligned}
& \left\|g_{n}(D ; h ; \chi ; \tau)\right\| \\
& \quad \leq C_{\epsilon, n}^{\chi_{\rho}+\tau_{\rho}} C_{\epsilon}^{\left(m_{\rho}\right)} C_{\epsilon}^{1-\chi_{\rho}+\ell_{\rho}-\tau_{\rho}} \mathrm{e}^{-\chi_{\rho} \lambda_{s}-\left(1-\chi_{\rho}\right) \lambda_{s+1}} \\
& \quad \exp \left(h_{\rho}\left(\lambda_{s+1}-\lambda_{s} \ell_{\rho}+\epsilon\left(3+2 \ell_{\rho}\right)\right)\right) \prod_{i=1}^{r_{\rho}}\left\|g_{n, h_{\rho}}\left(D_{\rho_{i}} ; h_{\mid D_{\rho_{i}}} ; \chi_{\mid D_{\rho_{i}}} ; \tau_{\mid D_{\rho_{i}}}\right)\right\| .
\end{aligned}
$$

Inequalities $(26)$ and $(27)$ and the fact that $(\chi, \tau) \in \mathcal{Q}(D)$ provide the first inequality for $\left\|g_{n}(D ; h ; \chi ; \tau)\right\|$.

To deduce the second inequality, it suffices to note that when $u \in \mathfrak{i}_{2}(D), h_{p(u)}$ is smaller than $h_{u}$. This allows to bound $\lambda_{s+1}\left(h_{u}-h_{p(u)}\right)$ by $\lambda_{s}\left(h_{u}-h_{p(u)}\right)$ for $u \in \mathfrak{i}_{2}(D)$. In this way, a unique bound of $\left\|g_{n, h_{p(u)}}\left(D_{u} ; h_{\mid D_{u}} ; \chi_{\mid D_{u}} ; \tau_{\mid D_{u}}\right)\right\|$ is obtained for all $u \in \mathfrak{i}(D)$ :

$$
\begin{aligned}
& \left\|g_{n, h_{p(u)}}\left(D_{u} ; h_{\mid D_{u}} ; \chi_{\mid D_{u}} ; \tau_{\mid D_{u}}\right)\right\| \\
& \leq C_{\epsilon, n}^{\chi_{u}+\tau_{u}} C_{\epsilon}^{\left(m_{u}\right)} C_{\epsilon}^{1-\chi_{u}+\ell_{u}-\tau_{u}} \mathrm{e}^{-\lambda_{s}} \mathrm{e}^{h_{p(u)}}\left(-\lambda_{s}+2 \epsilon\right) \\
& \quad \exp \left(h_{u}\left(\lambda_{s}\left(1-\ell_{u}\right) \tau_{u}+\epsilon\left(3+2 \ell_{u}\right)\right)\right) \\
& \prod_{i=1}^{r_{u}}\left\|g_{n, h_{u}}\left(D_{u_{i}} ; h_{\mid D_{u_{i}}} ; \chi_{\mid D_{u_{i}}} ; \tau_{\mid D_{u_{i}}}\right)\right\| .
\end{aligned}
$$

In the same way,

$$
\begin{aligned}
\left\|g_{n}(D ; h ; \chi ; \tau)\right\| \leq & C_{\epsilon, n}^{\chi_{\rho}+\tau_{\rho}} C_{\epsilon}^{\left(m_{\rho}\right)} C_{\epsilon}^{1-\chi_{\rho}+\ell_{\rho}-\tau_{\rho}} \mathrm{e}^{-\chi_{\rho} \lambda_{s}-\left(1-\chi_{\rho}\right) \lambda_{s+1}} \\
& \exp \left(h_{\rho}\left(\lambda_{s}\left(1-\ell_{\rho}\right)+\epsilon\left(3+2 \ell_{\rho}\right)\right)\right) \\
& \prod_{i=1}^{r_{\rho}}\left\|g_{n, h_{\rho}}\left(D_{\rho_{i}} ; h_{\mid D_{\rho_{i}}} ; \chi_{\mid D_{\rho_{i}}} ; \tau_{\mid D_{\rho_{i}}}\right)\right\| .
\end{aligned}
$$

BULLETIN DE LA SOCIÉtÉ MATHÉMATIQUE DE FRANCE 
In Subsection 3.2, it has been shown that for a positive and small enough $\epsilon$

- $\sum_{h \in H_{n}(D)} \exp \left(\sum_{u \in \mathfrak{i}(D)} h_{u} \tilde{\beta}_{u}\right)$ converges if $\lambda_{s}>0$;

- $\sum_{h \in H_{n}(D)} \exp \left(\sum_{u \in \mathfrak{i}(D)} h_{u} \beta_{u}\right)$ converges if $\lambda_{s} \leq 0$ and $\lambda_{s+1}-k \lambda_{s}<0$.

Therefore, according to Lemma 3.14 , for every $D \in \mathcal{T}_{k}$ and $(\chi, \tau) \in \mathcal{Q}(D)$, the sequence $\left(\sum_{h \in H_{n}(D)} g_{n}(D ; h ; \chi ; \tau)\right)_{n}$ converges to zero in probability if $\lambda_{s+1}-k \lambda_{s}<0$ and $D \xi(0)\left(\mathbb{R}^{d}\right) \cap \bigoplus_{i=s+1}^{r} E_{i}=\{0\} \mathbb{P}$-almost surely. This closes the proof of the convergence to 0 of $\left(F_{n+1}^{1}(2 ; D)(0)-F_{n}(1 ; D)(0)\right)_{n}$ for every diagram $D \in \mathcal{T}_{k}$.

3.3.2. Convergence to zero of $\left(F_{n+1}^{2}(2 ; D)(0)\right)_{n}$. - Let $D \in \mathcal{T}_{k}$. To prove that the sequence $\left(F_{n+1}^{2}(2 ; D)(0)\right)_{n}$ converges to 0 in probability, let us introduce an auxiliary sequence

$$
\tilde{F}_{n+1}(D)=\sum_{h \in H_{n+1}(D) \backslash H_{n}(D)} \tilde{f}_{n+1}(D ; h),
$$

that can be deduced from the expression of $F_{n+1}^{2}(2, D)(0)$ by replacing each projector $\Pi_{n}$ by a projector $\Pi$ :

$$
\begin{aligned}
\tilde{f}_{n+1}(D ; h)=(\mathrm{I}-\Pi) A_{h_{\rho}-1}\left(\theta^{-\left(h_{\rho}-1\right)}\right) b_{h_{\rho}}^{\left(m_{\rho}\right)}(2)(0) & \\
& \left(\tilde{f}_{n+1, h_{\rho}}\left(D_{\rho_{1}} ; h_{\mid D_{\rho_{1}}}\right), \ldots, \tilde{f}_{n+1, h_{\rho}}\left(D_{\rho_{r}} ; h_{\mid D_{\rho_{r}}}\right),\left(A_{-h_{\rho}} \Pi\right)^{\ell_{\rho}}\right)
\end{aligned}
$$

where $\rho_{1}, \ldots, \rho_{r_{\rho}}$ designate the non-terminal sons of the root $\rho$ and,

- if $u \in \mathfrak{i}_{1}(D)$ then

$$
\begin{aligned}
\tilde{f}_{n+1, h_{p(u)}} & \left(D_{u} ; h_{\mid D_{u}}\right)=A_{-h_{p(u)}} \Pi A_{h_{u}-1}\left(\theta^{-\left(h_{u}-1\right)}\right) b_{h_{u}}^{\left(m_{u}\right)}(2)(0) \\
& \left(\tilde{f}_{n+1, h_{u}}\left(D_{u_{1}} ; h_{\mid D_{u_{1}}}\right), \ldots, \tilde{f}_{n+1, h_{u}}\left(D_{u_{r}} ; h_{\mid D_{u_{r}}}\right),\left(A_{-h_{u}} \Pi\right)^{\otimes \ell_{u}}\right) .
\end{aligned}
$$

- if $u \in \mathfrak{i}_{2}(D)$ then

$$
\begin{array}{r}
\tilde{f}_{n+1, h_{p(u)}}\left(D_{u} ; h_{\mid D_{u}}\right)=\tilde{A}_{h_{p(u)}, h_{u}-1} b_{h_{u}}^{\left(m_{u}\right)}(2)(0)\left(\tilde{f}_{n+1, h_{u}}\left(D_{u_{1}} ; h_{\mid D_{u_{1}}}\right)\right. \\
\left.\ldots, \tilde{f}_{h_{u}}\left(D_{u_{r}} ; h_{\mid D_{u_{r}}}\right),\left(A_{-h_{u}} \Pi\right)^{\otimes \ell_{u}}\right) .
\end{array}
$$

where $u_{1}, \ldots, u_{r_{u}}$ designate the non-terminal sons of the node $u$.

The convergence to zero of the sequences $\left(\tilde{F}_{n}(D)\right)_{n}$ and $\left(\tilde{F}_{n}(D)-\right.$ $\left.F_{n}^{2}(2 ; D)(0)\right)_{n}$ will be successively studied.

3.3.2.1. Convergence of $\left(\tilde{F}_{n}(D)\right)_{n}$ to zero. — The expressions of $f_{n+1}(1 ; D ; h)(0)$ and $\tilde{f}_{n+1}(D ; h)$ only differ in the terms

$$
b_{n+1}^{(m)}(i)(0)= \begin{cases}B^{(m)}\left(\theta^{-(n+1)}\right) & \text { if } i=1, \\ \xi^{(m)}\left(\theta^{-n}\right) & \text { if } i=2 .\end{cases}
$$

Therefore, as in Lemmas 3.9, one may assert that for all $\epsilon>0$, there exists a $\mathbb{P}$-almost surely finite random variable $C_{\epsilon}$, such that for all $n \in \mathbb{N}^{*}$ and

TOME $129-2001-\mathrm{N}^{\mathrm{O}} 3$ 
$h \in H_{n}(D)$,

$$
\left\|\tilde{f}_{n}(D ; h)\right\| \leq\left(\prod_{u \in \mathfrak{i}(D), h_{u}=n}\left\|\xi^{\left(m_{u}\right)}\left(\theta^{-n}\right)\right\|\right) C_{\epsilon} \exp \left(\sum_{u \in \mathfrak{i}(D)} \alpha_{u} h_{u}\right)
$$

where

- $\alpha_{u}=\lambda_{s}\left(1-m_{u}\right)+\epsilon\left(2+m_{u}\right)$ if $u \in \mathfrak{i}(D)$ and $\lambda_{s}>0$;

- $\alpha_{u}=-\lambda_{s}\left(\ell_{u}+r_{u}^{(1)}\right)+\left(1-r_{u}^{(2)}\right) \lambda_{s+1}+\epsilon\left(2+m_{u}\right)$ if $u \in \mathfrak{i}_{2}(D) \cup\{\rho(D)\}$ and $\lambda_{s} \leq 0$

- $\alpha_{u}=\lambda_{s}\left(1-\ell_{u}-r_{u}^{(1)}\right)-r_{u}^{(2)} \lambda_{s+1}+\epsilon\left(2+m_{u}\right)$ if $u \in \mathfrak{i}_{1}(D)$ and $\lambda_{s} \leq 0$.

Therefore, to prove that $\left(\tilde{F}_{n+1}(D)\right)_{n}$ converges to zero in probability, it suffices to show that the sequence $\left(\sum_{h \in H_{n+1}(D) \backslash H_{n}(D)} \exp \left(\sum_{u \in \mathfrak{i}(D)} \alpha_{u} h_{u}\right)\right)_{n}$ converges to zero.

Set $J(D ; u ; n):=\left\{h \in H(D), h_{u}=n\right\}$ for $u \in \mathfrak{i}(D)$. According to Lemmas 3.10 and 3.13, whether $\lambda_{s}>0$ or $\lambda_{s} \leq 0$, the sum $\sum_{h \in H(D)} \exp \left(\sum_{u \in \mathfrak{i}(D)} \alpha_{u} h_{u}\right)$ is finite provided that $\lambda_{s+1}-k \lambda_{s}<0$ and that $\epsilon>0$ is small enough. Therefore, for every $u \in \mathfrak{i}(D)$, the sequence $(S(u ; n))_{n}$ defined by

$$
S(u ; n):=\sum_{h \in J(D ; u ; n)} \exp \left(\sum_{v \in \mathfrak{i}(D) \backslash\{u\}} h_{v} \alpha_{v}+n \alpha_{u}\right),
$$

converges to zero. But $H_{n+1}(D) \backslash H_{n}(D) \subset \bigcup_{u \in \mathfrak{i}(D)} J(D ; u ; n+1)$, whence the sequence $\left(\sum_{h \in H_{n+1}(D) \backslash H_{n}(D)} \exp \left(\sum_{u \in \mathfrak{i}(D)} \alpha_{u} h_{u}\right)\right)_{n}$ converges to zero.

3.3.2.2. Convergence of $\left(F_{n}^{2}(2 ; D)(0)-\tilde{F}_{n}(D)\right)_{n}$ to zero. - The difference between $F_{n+1}^{2}(2 ; D)(0)$ and $\tilde{F}_{n+1}(D)$ can also be decomposed as follows:

$$
F_{n+1}^{2}(2 ; D)(0)-\tilde{F}_{n+1}(D)=\sum_{(\chi, \tau) \in \mathcal{Q}(D)} \beta(\chi, \tau) \sum_{h \in H_{n}(D)} \tilde{g}_{n}(D ; h ; \chi ; \tau)
$$

where $\tilde{g}_{n}(D ; h ; \chi ; \tau)$ is the multilinear application defined by

$$
\begin{gathered}
\tilde{g}_{n}(D ; h ; \chi ; \tau)=\bar{\kappa}_{n}\left(\chi_{\rho}\right) A_{h_{\rho}-1}\left(\theta^{-\left(h_{\rho}-1\right)}\right) b_{h_{\rho}}^{\left(m_{\rho}\right)}(2)(0) \\
\left(\tilde{g}_{n, h_{\rho}}\left(D_{\rho_{1}} ; h_{\mid D_{\rho_{1}}} ; \chi_{\mid D_{\rho_{1}}} ; \tau_{\mid D_{\rho_{1}}}\right), \ldots, \tilde{g}_{n, h_{\rho}}\left(D_{\rho_{r_{\rho}}} ; h_{\mid D_{\rho_{r_{\rho}}}} ; \chi_{\mid D_{\rho_{r_{\rho}}}} ; \tau_{\mid D_{\rho_{r_{\rho}}}}\right)\right. \\
\left.\left(A_{-h_{\rho}}\left(\Pi_{n}-\Pi\right)\right)^{\otimes \tau_{\rho}},\left(A_{-h_{\rho}} \Pi\right)^{\otimes\left(\ell_{\rho}-\tau_{\rho}\right)}\right)
\end{gathered}
$$

$\left(\rho_{1}, \ldots, \rho_{r_{\rho}}\right.$ are the non-terminal sons of the root $\left.\rho\right)$. As usual, if $u \in \mathfrak{i}_{1}(D)$ then the map $\tilde{g}_{n, z}\left(D_{u} ; h_{\mid D_{u}} ; \chi_{\mid D_{u}} ; \tau_{\mid D_{u}}\right)$ is defined by:

$$
\begin{aligned}
& \tilde{g}_{n, h_{p(u)}}\left(D_{u} ; h_{\mid D_{u}} ; \chi_{\mid D_{u}} ; \tau_{\mid D_{u}}\right)=A_{-h_{p(u)}} \bar{e}_{n}\left(\chi_{u}\right) A_{h_{u}-1}\left(\theta^{-\left(h_{u}-1\right)}\right) b_{h_{u}}^{\left(m_{u}\right)}(2)(0) \\
& \left(\tilde{g}_{n, h_{u}}\left(D_{u_{1}} ; h_{\mid D_{u_{1}}} ; \chi_{\mid D_{u_{1}}} ; \tau_{\mid D_{u_{1}}}\right), \ldots, \tilde{g}_{n, h_{u}}\left(D_{u_{r_{u}}} ; h_{\mid D_{u_{r_{u}}}} ; \chi_{\mid D_{u_{r_{u}}}} ; \tau_{\mid D_{u_{r_{u}}}}\right)\right. \text {, } \\
& \left.\left(A_{-h_{u}}\left(\Pi_{n}-\Pi\right)\right)^{\otimes \tau_{u}},\left(A_{-h_{u}} \Pi\right)^{\otimes\left(\ell_{u}-\tau_{u}\right)}\right)
\end{aligned}
$$

BULLETIN DE LA SOCIÉTÉ MATHÉMATIQUE DE FRANCE 
and if $u \in \mathfrak{i}_{2}(D)$ then the map $\tilde{g}_{n, z}\left(D_{u} ; h_{\mid D_{u}} ; \chi_{\mid D_{u}} ; \tau_{\mid D_{u}}\right)$ is defined by:

$$
\begin{gathered}
\tilde{g}_{n, h_{p(u)}}\left(D_{u} ; h_{\mid D_{u}} ; \chi_{\mid D_{u}} ; \tau_{\mid D_{u}}\right)=\bar{a}_{h_{p(u)}, h_{u}-1}\left(\chi_{u}\right) b_{h_{u}}^{\left(m_{u}\right)}(2)(0) \\
\left(\tilde{g}_{n, h_{u}}\left(D_{u_{1}} ; h_{\mid D_{u_{1}}} ; \chi_{\mid D_{u_{1}}} ; \tau_{\mid D_{u_{1}}}\right), \ldots, \tilde{g}_{n, h_{u}}\left(D_{u_{r_{u}}} ; h_{\mid D_{u_{r_{u}}}} ; \chi_{\mid D_{u_{r_{u}}}} ; \tau_{\mid D_{u_{r_{u}}}}\right),\right. \\
\left.\left(A_{-h_{u}}\left(\Pi_{n}-\Pi\right)\right)^{\otimes \tau_{u}},\left(A_{-h_{u}} \Pi\right)^{\otimes\left(\ell_{u}-\tau_{u}\right)}\right)
\end{gathered}
$$

$\left(u_{1}, \ldots, u_{r_{u}}\right.$ designate the non-terminal sons of the node $\left.u\right)$.

For $0<\epsilon<\lambda_{s}-\lambda_{s+1},(\chi, \tau) \in \mathcal{Q}(D)$ and $h \in H_{n}(D)$, the norm of the application $\tilde{g}_{n}(D ; \chi ; \tau ; h)$ is bounded above by

$$
\left\|\tilde{g}_{n}(D ; \chi ; \tau ; h)\right\| \leq C_{\epsilon, n} \prod_{u \in \mathfrak{i}(D), h_{u}=n}\left\|\xi^{\left(m_{u}\right)}\left(\theta^{-n}\right)\right\| \exp \left(\sum_{u \in \mathfrak{i}(D)} \beta_{u} h_{u}\right)
$$

where

- $\beta_{u}=\lambda_{s}\left(1-m_{u}\right)+\epsilon\left(3+2 m_{u}\right)$ if $u \in \mathfrak{i}(D)$ and $\lambda_{s}>0$;

- $\beta_{u}=-\lambda_{s}\left(\ell_{u}+r_{u}^{(1)}\right)+\left(1-r_{u}^{(2)}\right) \lambda_{s+1}+\epsilon\left(3+2 m_{u}\right)$ if $u \in \mathfrak{i}_{2}(D) \cup\{\rho(D)\}$ and $\lambda_{s} \leq 0$

- $\beta_{u}=\lambda_{s}\left(1-\ell_{u}-r_{u}^{(1)}\right)-r_{u}^{(2)} \lambda_{s+1}+\epsilon\left(3+2 m_{u}\right)$ if $u \in \mathfrak{i}_{1}(D)$ and $\lambda_{s} \leq 0$;

- $\left(C_{\epsilon, n}\right)_{n}$ is a sequence of random variables that converges in probability to zero.

When $\lambda_{s+1}-k \lambda_{s}<0$, and $\epsilon>0$ is small enough, the sum

$$
\sum_{h \in H(D)} \exp \left(\sum_{u \in \mathfrak{i}(D)} \beta_{u} h_{u}\right)
$$

is finite. Therefore, one may conclude that

$$
\left(\sum_{h \in H_{n+1}(D) \backslash H_{n}(D)}\left\|\tilde{g}_{n}(D ; \chi ; \tau ; h)\right\|\right)_{n}
$$

converges to zero in probability for every $D \in \mathcal{T}_{k}$ and $(\chi, \tau) \in \mathcal{Q}(D)$.

This closes the proof of the convergence to zero in probability of $\left(D^{k} \Gamma_{n}(0)-D^{k} \Upsilon_{n}(0)\right)$ when $\lambda_{s+1}-k \lambda_{s}<0$ and $D \xi(0)\left(\mathbb{R}^{d}\right) \cap \bigoplus_{i=s+1}^{r} E_{i}=\{0\}$ $\mathbb{P}$-almost surely.

\section{Appendix A}

\section{Description of the contacts of submanifolds}

The objective of this appendix is to introduce equivalence classes of submanifolds that play the same role as jets for maps and to state some of their elementary properties.

TOME $129-2001-\mathrm{N}^{\mathrm{O}} 3$ 
A.1. Properties of the jets of maps. - Let us start by recalling some properties of the jets of maps.

Lemma A.1. - Let E, $F$ and $G$ be three Euclidean spaces.

1) There exists a unique application, Co, defined on the product

$$
J_{0}^{k}\left(\mathcal{C}_{0,0}^{\infty}(E, F)\right) \times J_{0}^{k}\left(\mathcal{C}_{0,0}^{\infty}(F, G)\right)
$$

such that for all $f \in \mathcal{C}_{0,0}^{\infty}(E, F)$ and $g \in \mathcal{C}_{0,0}^{\infty}(F, G)$,

$$
\operatorname{Co}\left(j_{0}^{k}(f), j_{0}^{k}(g)\right)=j_{0}^{k}(g \circ f) .
$$

Furthermore, Co is continuous.

2) Denote by $\operatorname{Diff}_{0}^{\infty}(E)$ the set of local diffeomorphisms of $\mathcal{C}_{0,0}^{\infty}(E, E)$. There exists a unique map, Inv, defined on $J_{0}^{k}\left(\operatorname{Diff}_{0}^{\infty}(E)\right)$ such that for all $f$ in $\operatorname{Diff}_{0}^{\infty}(E)$,

$$
\operatorname{Inv}\left(j_{0}^{k}(f)\right)=j_{0}^{k}\left(f^{-1}\right) .
$$

Furthermore, Inv is continuous.

Proof. — These two properties are a consequence of Faa-di-Bruno's formula:

Let $E, F$ and $G$ be three Euclidean spaces, let $f$ be a $C^{\infty}$ map defined on a neighbourhood $U$ of 0 in $E$ with values in $F$, let $V$ be an open set of $F$ such that $f(U) \subset V$ and let $g: V \rightarrow G$ be a $C^{\infty}$ map. The map $g \circ f$ is $C^{\infty}$ on $U$ and for all $k \in \mathbb{N}^{*}, x \in U$

$$
D^{k}(f \circ g)(x)=\sum_{q \in I_{k}} \mathrm{C}_{k}^{q} D^{|q|} f(g(x))\left((D g(x))^{\otimes q_{1}}, \ldots,\left(D^{k} g(x)\right)^{\otimes q_{k}}\right),
$$

where

$$
\begin{aligned}
I_{k} & =\left\{q=\left(q_{1}, \ldots, q_{k}\right) \in \mathbb{N}^{k}, q_{1}+2 q_{2} \cdots+k q_{k}=k\right\} \\
|q| & =q_{1}+\cdots+q_{k}, \\
\mathrm{C}_{k}^{q} & =\frac{k !}{q_{1} ! \ldots q_{k} !} \cdot \frac{1}{(2 !)^{q_{2}} \ldots(k !)^{q_{k}}} \quad \text { for } \quad q=\left(q_{1}, \ldots, q_{k}\right) .
\end{aligned}
$$

By this formula, if $\tilde{f} \in j_{0}^{k}(f)$ and $\tilde{g} \in j_{0}^{k}(g)$, then $\tilde{g} \circ \tilde{f} \in j_{0}^{k}(g \circ f)$. Therefore, there exists a unique map Co, defined on $J_{0}^{k}\left(C_{0,0}^{\infty}(E, F)\right) \times J_{0}^{k}\left(C_{0,0}^{\infty}(F, G)\right)$, such that for all $f \in C_{0,0}^{\infty}(E, F)$ and $g \in C_{0,0}^{\infty}(F, G), j_{0}^{k}(g \circ f)=\operatorname{Co}\left(j_{0}^{k}(f), j_{0}^{k}(g)\right)$. Faa-di-Bruno's formula also shows that the map Co is continuous.

If $f \in \operatorname{Diff}_{0}^{\infty}(E)$, then Faa-di-Bruno's formula gives an iterative formula for the derivatives of $f^{-1}$ at $0: D f^{-1}(0)=(D f(0))^{-1}$ and for each $k \geq 2$, $D^{k} f^{-1}(0)$ is equal to

$$
-\sum_{q \in \tilde{I}_{k}} \mathrm{C}_{k}^{q} D^{|q|} f^{-1}(0)\left(\left(D f(0) D f^{-1}(0)\right)^{\otimes q_{1}}, \ldots,\left(D^{k} f(0)\left(D f^{-1}(0)^{\otimes k}\right)^{\otimes q_{k}}\right)\right)
$$

BULLETIN DE LA SOCiÉtÉ MATHÉMATIQUe DE FRANCE 
where

$$
\tilde{I}_{k}=\left\{q=\left(q_{1}, \ldots, q_{k}\right) \in I_{k},|q|<k\right\} .
$$

Thus, if $\tilde{f} \in j_{0}^{k}(f)$ then $\tilde{f}^{-1} \in j_{0}^{k}\left(f^{-1}\right)$; there exists a unique map Inv, defined on $J_{0}^{k}\left(\operatorname{Diff}_{0}^{\infty}(E)\right)$ such that for every $f \in \operatorname{Diff}_{0}^{\infty}(E), j_{0}^{k}\left(f^{-1}\right)=\operatorname{Inv}\left(j_{0}^{k}(f)\right)$. The iterative formula shows that Inv is continuous.

A.2. Orthogonal parametrization of a submanifold at $\mathbf{0}$. - This subsection contains a description of a particular class of parametrizations for submanifolds of $\mathbb{R}^{N}$.

Definition A.2. - Let $S$ be a $C^{\infty}$ submanifold of $\mathbb{R}^{N}$ passing through 0 and let $f \in C_{0,0}^{\infty}\left(T_{0} S, \mathbb{R}^{N}\right)$ be a parametrization of $S$ at 0 . " $f$ is an orthogonal parametrization of $S$ at 0 " if $f \in C_{0}^{\infty}\left(T_{0} S, \mathbb{R}^{N}\right)$ and if the orthogonal projection of $f$ on the tangent space $T_{0} S$ of $S$ at 0 is equal to the identity on $T_{0} S$.

For example, consider the unit circle $S=\left\{(x, y) \in \mathbb{R}^{2},(x+1)^{2}+y^{2}=1\right\}$. Its tangent space at 0 is the vector space $\mathbb{R}(0,1)$. The map $\gamma$, defined by $\gamma((0, t))=\left(\sqrt{1-t^{2}}-1, t\right)$ for all $\left.t \in\right]-1,1[$, is an orthogonal parametrization of $S$ at 0 .

An orthogonal parametrization of $S$ at 0 can be constructed with the help of a parametrization $f \in C_{0,0}^{\infty}\left(E, \mathbb{R}^{N}\right)$ of $S$ at 0 as follows: let $p$ denote the orthogonal projection on $T_{0} S$. There exist a neighbourhood $U$ of 0 in $E$ and a neighbourhood $V$ of 0 in $T_{0} S$, such that $p \circ f$ is a diffeomorphism from $U$ onto $V$. Then the map $g$, defined on $V$ by $g(y):=f \circ(p \circ f)^{-1}(y)$ for all $y \in V$, is an orthogonal parametrization of $S$ at 0 . The map $g \circ p \in C_{0}^{\infty}\left(\mathbb{R}^{N}, \mathbb{R}^{N}\right)$ will be called the "orthogonal normalization of $f$ " and will be denoted by $n_{\perp}(f)$.

The following lemma states two properties of orthogonal normalizations:

Lemma A.3. - Let $E$ be a subspace of $\mathbb{R}^{N}$ and let $g_{1}, g_{2} \in \operatorname{Emb}_{0,0}^{\infty}\left(E, \mathbb{R}^{N}\right)$.

1) If $g_{1}$ and $g_{2}$ are two parametrizations of a $C^{\infty}$ submanifold $S$ of $\mathbb{R}^{N}$ at 0 , then their orthogonal normalizations coincide on a neighbourhood of 0 in $\mathbb{R}^{N}$.

2) There exists a unique map $N_{\perp}$, defined on $J_{0}^{k}\left(\operatorname{Emb}_{0,0}^{\infty}\left(E, \mathbb{R}^{N}\right)\right)$, such that for all $f \in \operatorname{Emb}_{0,0}^{\infty}\left(E, \mathbb{R}^{N}\right), N_{\perp}\left(j_{0}^{k}(f)\right)=j_{0}^{k}\left(n_{\perp}(f)\right)$. The map $N_{\perp}$ is continuous.

Proof. - 1) Let $p$ be the orthogonal projection of $\mathbb{R}^{N}$ onto $T_{x} S$. There exists a neighbourhood $U$, of 0 in $E$ such that:

- $g_{1}$ and $g_{2}$ are homeomorphisms from $U$ onto their images $V_{1} \cap S$ and $V_{2} \cap S$, where $V_{1}$ and $V_{2}$ are two neighbourhoods of 0 in $\mathbb{R}^{N}$;

- $p \circ g_{1}$ and $p \circ g_{2}$ are diffeomorphisms from $U$ onto their images.

TOME $129-2001-\mathrm{N}^{\mathrm{O}} 3$ 
Set $V=V_{1} \cap V_{2}, U_{i}=g_{i}^{-1}(U)$ for $i \in\{1,2\}$, and $W=p^{-1}\left(U_{1} \cap U_{2}\right)$. There exists a homeomorphism $h: U_{1} \mapsto U_{2}$, such that $g_{2} \circ h=g_{1}$ on $U_{1}$. Thus, the orthogonal normalizations of $g_{1}$ and $g_{2}$ are well-defined on $W$ and

$$
n_{\perp}\left(g_{1}\right)=g_{2} \circ h \circ\left(p \circ g_{2} \circ h\right)^{-1} \circ p=n_{\perp}\left(g_{2}\right) .
$$

2) Due to Lemma A.1, it remains to show that there exists a unique map $P$, defined on $J_{0}^{k}\left(C_{0,0}^{\infty}\left(E, \mathbb{R}^{N}\right)\right)$ satisfying $j_{0}^{k}(p(f))=P\left(j_{0}^{k}(f)\right)$ for all $f$ in $\operatorname{Emb}_{0,0}^{\infty}\left(E, \mathbb{R}^{N}\right)$, and to prove that $P$ is continuous.

First, if $\tilde{f}, f \in \operatorname{Emb}_{0,0}^{\infty}\left(E, \mathbb{R}^{N}\right)$ have a contact of order at least one, then $p(f)=p(\tilde{f})$. Therefore $P$ is well-defined. The fact that $P$ is continuous will be established by proving that if $f, \tilde{f} \in \operatorname{Emb}_{0,0}^{\infty}\left(E, \mathbb{R}^{N}\right)$ satisfy

$$
\|D f(0)-D \tilde{f}(0)\|<1,
$$

then $\|p(f)-p(\tilde{f})\| \leq\|D f(0)-D \tilde{f}(0)\|<1$. Actually, according to the following theorem, it suffices to prove that $\|(\mathrm{I}-p(\tilde{f})) p(f)\| \leq\|D f(0)-D \tilde{f}(0)\|$.

Theorem A.4 (T. Kato [4]). - Let $P$ and $Q$ be two orthogonal projections in an Euclidean space. Set

$$
\delta(P, Q):=\max \{|\langle x, y\rangle|, x \in \operatorname{Im}(P), y \in \operatorname{Im}(Q) \text { and }\|x\|=\|y\|=1\} .
$$

If $\operatorname{dim} \operatorname{Im}(P)=\operatorname{dim} \operatorname{Im}(Q)$ and $\delta(1-P, Q)<1$, then $\|P-Q\|=\delta(1-P, Q)$.

Let us note that $\delta(P, Q)=\|P Q\|=\|Q P\|$.

Let $\left(e_{1}, \ldots, e_{N}\right)$ be an orthonormal basis of $\mathbb{R}^{N}$ such that $\left(e_{1}, \ldots, e_{d}\right)$ is a basis of $F=D f(0)(E)$. Set $\epsilon_{i}=D f^{-1}(0) e_{i}$ for $i \in\{1, \ldots, d\}$. For a point $x$ in $\mathbb{R}^{N}$ :

$$
\begin{array}{r}
\left\|D \tilde{f}(0) \epsilon_{1} \wedge \cdots \wedge D \tilde{f}(0) \epsilon_{d} \wedge p(f)(x)\right\|=\left\|D \tilde{f}(0) \epsilon_{1} \wedge \cdots \wedge D \tilde{f}(0) \epsilon_{d}\right\| \\
\|(I-p(\tilde{f})) p(f)(x)\| .
\end{array}
$$

The decomposition $p(f)(x)=\sum_{i=1}^{d}\left\langle x, e_{i}\right\rangle D f(0) \epsilon_{i}$, gives:

$$
\begin{aligned}
& \left\|D \tilde{f}(0) \epsilon_{1} \wedge \cdots \wedge D \tilde{f}(0) \epsilon_{d} \wedge p(f)(x)\right\| \leq\left\|D \tilde{f}(0) \epsilon_{1} \wedge \cdots \wedge D \tilde{f}(0) \epsilon_{d}\right\| \\
& \left\|\sum_{i=1}^{d}\left\langle x, e_{i}\right\rangle\left(D f(0) \epsilon_{i}-D \tilde{f}(0) \epsilon_{i}\right)\right\| .
\end{aligned}
$$

It turns out that $\|(\mathrm{I}-p(\tilde{f})) p(f)\| \leq\|D f(0)-D \tilde{f}(0)\|$.

A.3. The $k$-jets of submanifolds of $\mathbb{R}^{N}$ at 0 . - As a consequence of Lemma A.3, one may introduce equivalence classes of submanifolds of $\mathbb{R}^{N}$ at 0 .

Lemma A.5. - Let $k$ be a positive integer.

BULLETIN DE LA SOCiÉtÉ MATHÉMATIQUe DE FRANCE 
1) Consider two submanifolds of $\mathbb{R}^{N}$ passing through 0 denoted by $S_{1}$ and $S_{2}$. The following two assertions are equivalent:

(i) There exist an integer $d \in\{1, \ldots, N\}$ and two applications $f_{1}, f_{2} \in \operatorname{Emb}_{0,0}^{\infty}\left(\mathbb{R}^{d}, \mathbb{R}^{N}\right)$ such that $f_{1}$ and $f_{2}$ are parametrizations at 0 of $S_{1}$ and $S_{2}$ respectively, and $j_{0}^{k}\left(f_{1}\right)=j_{0}^{k}\left(f_{2}\right)$.

(ii) The orthogonal normalization of any parametrization of $S_{1}$ and of $S_{2}$ have the same $k$-jet at 0 .

2) The relation on the set of $C^{\infty}$ submanifolds of $\mathbb{R}^{N}$ passing through 0 defined by, " $S_{1}$ and $S_{2}$ have a contact of order at least $k$ at 0 if $S_{1}$ and $S_{2}$ satisfy (i)", is an equivalence relation.

The equivalence class of a $C^{\infty}$ submanifold $S$ of $\mathbb{R}^{N}$ at 0 for this equivalence relation is denoted $j_{0}^{k}(S)$ and called the $k$-jet of $S$ at 0 . Let $\mathcal{J}_{0}^{k}\left(\mathbb{R}^{N}\right)$ denote the set of all $k$-jets at 0 of $C^{\infty}$ submanifolds of $\mathbb{R}^{N}$. The metric $d_{k}$ on the set $J_{0}^{k}\left(C_{0,0}^{\infty}\left(\mathbb{R}^{N}, \mathbb{R}^{N}\right)\right)$ yields a metric on $\mathcal{J}_{0}^{k}\left(\mathbb{R}^{N}\right)$ denoted by $\partial_{k}$ :

Definition A.6. - Let $S_{1}$ and $S_{2}$ be two $C^{\infty}$ submanifolds of $\mathbb{R}^{N}$ passing through 0. For $i \in\{1,2\}$, let $f_{i}$ denote an orthogonal parametrization of $S_{i}$ at 0 and let $p_{i}$ designate the orthogonal projection onto $T_{0} S_{i}$. The distance between the $k$-jets of $S_{1}$ and $S_{2}$ at 0 is defined by

$$
\partial_{k}\left(j_{0}^{k}\left(S_{1}\right), j_{0}^{k}\left(S_{2}\right)\right):=d_{k}\left(j_{0}^{k}\left(f_{1} \circ p_{1}\right), j_{0}^{k}\left(f_{2} \circ p_{2}\right)\right) .
$$

LEMmA A.7. - The metric space $\left(\mathcal{J}_{0}^{k}\left(\mathbb{R}^{N}\right), \partial_{k}\right)$ is complete. For each $d$ in $\{1, \ldots, N\}$, the set of all $k$-jets at 0 of d-dimensional submanifolds of $\mathbb{R}^{N}$ is a closed subset of $\left(\mathcal{J}_{0}^{k}\left(\mathbb{R}^{N}\right), \partial_{k}\right)$.

Proof. - Let $\left(j_{0}^{k}\left(S_{n}\right)\right)_{n}$ be a Cauchy sequence, let $\left(f_{n}\right)_{n}$ be the sequence of orthogonal parametrizations of the submanifolds $S_{n}$ at 0 and let $\left(p_{n}\right)_{n}$ be the sequence of orthogonal projections onto $T_{0} S_{n}$. Then, for all $j \in\{1, \ldots, k\}$, the sequence $\left(D^{j}\left(f_{n} \circ p_{n}\right)(0)\right)_{n}$ converges to a $j$-linear map denoted by $\alpha_{j}$. Set $P_{k}=\sum_{j=1}^{k} \alpha_{j} /(j !)$ and $E=\operatorname{Im}\left(\alpha_{1}\right)$. For every $n \in \mathbb{N}, D\left(f_{n} \circ p_{n}\right)(0)$ is equal to $p_{n}$ and $p_{n} \circ D^{j} f_{n}(0)$ vanishes on $T_{0} S_{n}$ for $j \in\{2, \ldots, k\}$. Therefore $\alpha_{1}$ is the orthogonal projection onto $E$ and $\alpha_{1} \circ P_{k}$ is the identity on $E$. In conclusion, there exists a neighbourhood $U$ of 0 in $E$ such that $P_{k}$ is a $C^{\infty}$ diffeomorphism from $U$ onto its image and the restriction of $P_{k}$ to $U$ is the orthogonal parametrization of the submanifold $S=P_{k}(U)$. This shows that $\left(j_{0}^{k}\left(S_{n}\right)\right)_{n}$ converges to $j_{0}^{k}(S)$.

Assume in addition that $S_{n}$ are $d$-dimensional submanifolds of $\mathbb{R}^{N}$. As the orthogonal projection $p_{n}$ onto $T_{0} S_{n}$ converges to the orthogonal projection onto $E$, then the dimension of $E$ is $d$.

REMARK A.8. - Let us note that if $\left(S_{n}\right)_{n}$ is a sequence of smooth submanifolds of $\mathbb{R}^{N}$ passing through 0 such that $\left(j_{0}^{k}\left(S_{n}\right)\right)_{n}$ converges to the $k$-jet of a 
submanifold $S$ at 0 , then the dimension of $T_{0} S_{n}$ is constant for $n$ large enough and equal to that of $T_{0} S$.

The following lemma gives another characterization of the convergence in $\mathcal{J}_{x}^{k}\left(\mathbb{R}^{N}\right)$ :

LEMmA A.9. - Let $S_{n}$, for all $n \in \mathbb{N}$, be $C^{\infty}$ d-dimensional submanifolds of $\mathbb{R}^{N}$ passing through 0 . The following two assertions are equivalent:

(i) There exist a map $f \in \operatorname{Emb}_{0,0}^{\infty}\left(\mathbb{R}^{d}, \mathbb{R}^{N}\right)$ and, for all $n \in \mathbb{N}$, a parametrization $f_{n} \in \operatorname{Emb}_{0,0}^{\infty}\left(\mathbb{R}^{d}, \mathbb{R}^{N}\right)$ of $S_{n}$ at 0 , such that $\left(j_{0}^{k}\left(f_{n}\right)\right)_{n}$ converges to $j_{0}^{k}(f)$.

(ii) $\left(j_{0}^{k}\left(S_{n}\right)\right)_{n}$ converges in $\left(\mathcal{J}_{0}^{k}\left(\mathbb{R}^{N}\right), \partial_{k}\right)$.

If assertion (i) holds, then there exists a neighbourhood $U$ of 0 in $\mathbb{R}^{d}$ such that $S:=f(U)$ is a d-dimensional submanifold and $\partial_{k}\left(j_{0}^{k}\left(S_{n}\right), j_{0}^{k}(S)\right)$ converges to 0 as $n$ tends to $\infty$.

Proof. - (i) $\Rightarrow$ (ii). The map $N_{\perp}$, defined on $J_{0}^{k}\left(\operatorname{Emb}_{0}^{\infty}\left(\mathbb{R}^{d}, \mathbb{R}^{N}\right)\right)$ by

$$
N_{\perp}\left(j_{0}^{k}(f)\right)=j_{0}^{k}\left(n_{\perp}(f)\right)
$$

for all $f \in \operatorname{Emb}_{0}^{\infty}\left(\mathbb{R}^{d}, \mathbb{R}^{N}\right)$, is continuous. Hence, (i) implies that the sequence $\partial_{k}\left(j_{0}^{k}\left(S_{n}\right), j_{0}^{k}(S)\right)$ converges to 0 as $n$ tends to $\infty$.

(ii) $\Rightarrow$ (i). For $n \in \mathbb{N}$, let $g_{n}$ denote the orthogonal parametrization of $S_{n}$ at 0 and let $p_{n}$ denote the orthogonal projection onto $T_{0} S_{n}$. Set $\alpha_{i}=\lim _{n \rightarrow+\infty} D^{i}\left(g_{n} \circ p_{n}\right)(0)$ and $P_{k}=\sum_{i=1}^{k} \alpha_{i} /(i !)$. In the proof of Lemma A.7, it has been shown that there exists a neighbourhood $U$ of 0 in $E=\operatorname{Im}\left(\alpha_{1}\right)$ such that $g:=P_{k \mid U}$ is the orthogonal parametrization of the submanifold $S=P_{k}(U)$. For all $n \in \mathbb{N}$, there exists a linear map $T_{n} \in O\left(\mathbb{R}^{N}\right)$ such that $T_{n}\left(\mathbb{R}^{d}\right)=T_{0} S_{n}$. One may suppose that the sequence $\left(T_{n}\right)_{n}$ converges to $T \in O\left(\mathbb{R}^{N}\right)$, otherwise one considers a subsequence. As $\left(p_{n}\right)$ converges to the orthogonal projection onto $T_{0} S, T\left(\mathbb{R}^{d}\right)=T_{0} S$. The restriction of $g \circ T$ and $g_{n} \circ T_{n}$ to a neighbourhood of 0 in $\mathbb{R}^{d}$ are parametrizations of $S$ and $S_{n}$ at 0 that belong to $\operatorname{Emb}_{0}^{\infty}\left(\mathbb{R}^{d}, \mathbb{R}^{N}\right)$. By Lemma A.1, the $k$-jet at 0 of $g_{n} \circ T_{n}$ converges to the $k$-jet at 0 of $g \circ T$.

A.4. Jets of submanifolds of a $C^{\infty}$ Riemannian manifold. - Let $\left(M,\langle\cdot, \cdot\rangle_{x}, x \in M\right)$ be a $C^{\infty}$ Riemannian manifold of dimension $N$. Equivalence classes of submanifolds of $M$ at a point $x$ can be defined by taking charts at $x$.

LEMmA A.10. - Let $S_{1}$ and $S_{2}$ be two submanifolds of $M$ passing through a point $x$.

1) If there exists a chart $(\zeta, U)$ of $M$ at $x$ such that the $k$-jets of $\zeta\left(S_{1} \cap U\right)$ and $\zeta\left(S_{2} \cap U\right)$ at $x$ are equal, then for any other chart $(\tilde{\zeta}, \tilde{U})$ of $M$ at $x$, the $k$-jets of $\tilde{\zeta}\left(S_{1} \cap \tilde{U}\right)$ and $\tilde{\zeta}\left(S_{2} \cap \tilde{U}\right)$ at $x$ are equal. 
2) The relation on the set of submanifolds of $M$ passing through $x$ defined by " $S_{1}$ and $S_{2}$ have a contact of order at least $k$ at $x$, if for every chart $(\zeta, U)$ of $M$ at $x, \zeta\left(S_{1} \cap U\right)$ and $\zeta\left(S_{2} \cap U\right)$ have a contact of order at least $k$ at $0 "$ is an equivalence relation.

Proof. - Let $(\zeta, U)$ and $(\tilde{\zeta}, \tilde{U})$ be two charts of $M$ at $x$. Assume that $j_{0}^{k}\left(\zeta\left(S_{1} \cap U\right)\right)=j_{0}^{k}\left(\zeta\left(S_{2} \cap U\right)\right)$. If $f_{i}$ is a parametrization of $\zeta\left(S_{1} \cap U\right)$ for $i \in\{1,2\}$ at 0 , then $\tilde{\zeta} \circ \zeta^{-1} \circ f_{i}$ is a parametrization of $\tilde{\zeta}\left(S_{1} \cap U \cap \tilde{U}\right)$ at 0 . Therefore, it suffices to use that $j_{0}^{k}\left(f_{1}\right)=j_{0}^{k}\left(f_{2}\right)$ implies

$$
j_{0}^{k}\left(\tilde{\zeta} \circ \zeta^{-1} \circ f_{1}\right)=j_{0}^{k}\left(\tilde{\zeta} \circ \zeta^{-1} \circ f_{2}\right) .
$$

The second statement is then trivial.

The class of a submanifold $S$ of $M$ for this equivalence relation is denoted by $j_{x}^{k}(S)$ and called the $k$-jet of $S$ at $x$. The set of $k$-jets at $x$ of all $C^{\infty}$ submanifolds of $M$ is denoted by $\mathcal{J}_{x}^{k}(M)$ and the union of the sets $\mathcal{J}_{x}^{k}(M)$ for all $x \in M$ is denoted by $\mathcal{J}^{k}(M)$.

If $M$ is the Euclidean space $\mathbb{R}^{N}$, then the simplest chart at a point $x$ is given by the translation by the vector $-x$ (denoted by $t_{-x}$ ). This shows that the contact between $S_{1}$ and $S_{2}$ at $x$ can still be defined with the help of parametrizations:

LEMMA A.11. - Let $x \in \mathbb{R}^{N}$ and let $S_{1}$ and $S_{2}$ be two d-dimensional submanifolds of $\mathbb{R}^{N}$ passing through $x . S_{1}$ and $S_{2}$ have a contact of order at least $k$ at $x$ if and only if there exist parametrizations of $S_{1}$ and $S_{2}$ at $x$ that belong to $\operatorname{Emb}_{0, x}\left(\mathbb{R}^{d}, \mathbb{R}^{N}\right)$ and have the same $k$-jet at 0 .

There are two ways to prove the convergence in $\mathcal{J}^{k}\left(\mathbb{R}^{N}\right)$ :

LEMmA A.12. - Let $\left(x_{n}\right)$ be a sequence of points of $\mathbb{R}^{N}$ and for every $n \in \mathbb{N}$, let $S_{n}$ be a d-dimensional submanifold of $\mathbb{R}^{N}$ passing through $x_{n}$. The following propositions (i) and (ii) are equivalent:

(i) for every $n \in \mathbb{N}$, there exists a parametrization $f_{n} \in \operatorname{Emb}_{0, x_{n}}^{\infty}\left(\mathbb{R}^{d}, \mathbb{R}^{N}\right)$ of $S_{n}$ at $x_{n}$ such that $\left(j_{x_{n}}^{k}\left(f_{n}\right)\right)_{n}$ converges;

(ii) $\left(x_{n}\right)_{n}$ converges and $\left(j_{0}^{k}\left(t_{-x_{n}}\left(S_{n}\right)\right)\right)_{n}$ converges in $\left(\mathcal{J}_{0}^{\infty}\left(\mathbb{R}^{N}\right), \partial_{k}\right)$.

If (i) and (ii) hold, the sequence $\left(j_{x_{n}}^{k}\left(S_{n}\right)\right)_{n}$ is said to converge.

Let us revert to the general case of a Riemannian manifold $M$. The following lemma assures that the convergence in $\mathcal{J}^{k}(M)$ can be defined by taking a chart:

LEMmA A.13. - Let $\left(x_{n}\right)_{n}$ be a sequence in $M$ and let, for every $n \in \mathbb{N}, S_{n}$ be a sequence of submanifolds of $M$ passing through $x_{n}$. Assume that $\left(x_{n}\right)_{n}$ converges to a point denoted by $x$. If there exists a chart $(\zeta, U)$ of $M$ at $x$ such that the $k$-jet of $\zeta\left(S_{n} \cap U\right)$ at $\zeta\left(x_{n}\right)$ converges, then there exists a submanifold

TOME $129-2001-\mathrm{N}^{\mathrm{O}} 3$ 
$S$ of $M$ at $x$ such that for any chart $(\zeta, U)$ of $M$ at $x$, the $k$-jet of $\zeta\left(S_{n} \cap U\right)$ at 0 converges to the $k$-jet of $\zeta(S \cap U)$ at 0 as $n$ tends to $+\infty$.

Proof. - Let $(\zeta, U)$ be a chart of $M$ at $x$ such that $\left(j_{\zeta\left(x_{n}\right)}^{k}\left(\zeta\left(S_{n} \cap U\right)\right)\right)_{n}$ converges. Let $S_{\zeta}$ denote a submanifold which belongs to the asymptotic $k$-jet. There exists a neighbourhood $V$ of 0 such that $S:=\zeta^{-1}\left(S_{\zeta} \cap V\right)$ defines a $C^{\infty}$ submanifold of $M$.

Let $(\tilde{\zeta}, \tilde{U})$ be another chart of $M$ at $x$. The convergence of

$$
\left(j_{\tilde{\zeta}\left(x_{n}\right)}^{k}\left(\tilde{\zeta}\left(S_{n} \cap \tilde{U}\right)\right)\right)_{n}
$$

to $j_{0}^{k}(\tilde{\zeta}(S \cap \tilde{U}))$ has to be proved. It follows from Remark A.8 that the submanifolds $S_{n}$, for all $n \in \mathbb{N}$, can be assumed to have the same dimension at $x_{n}$, denoted by $d$. There exists a parametrization $f_{n} \in \operatorname{Emb}_{0, \zeta\left(x_{n}\right)}^{\infty}\left(\mathbb{R}^{d}, \mathbb{R}^{N}\right)$ of $\zeta\left(S_{n}\right)$ at $\zeta\left(x_{n}\right)$ and a parametrization, $f \in \operatorname{Emb}_{0,0}^{\infty}\left(\mathbb{R}^{d}, \mathbb{R}^{N}\right)$, of $S_{\zeta}$ at $x$, such that $\left(j_{0}^{k}\left(f_{n}\right)\right)_{n}$ converges to $j_{0}^{k}(f)$. The map $\tilde{\zeta} \circ \zeta^{-1} \circ f_{n}$ belongs to $\operatorname{Emb}_{0, \tilde{\zeta}\left(x_{n}\right)}^{\infty}$ and is a parametrization of $\tilde{\zeta}\left(S_{n}\right)$ at $\tilde{\zeta}\left(x_{n}\right)$. As $\left(D^{j}\left(\tilde{\zeta} \circ \zeta^{-1}\right)\left(\zeta\left(x_{n}\right)\right)\right)_{n}$ converges to $D^{j}\left(\tilde{\zeta} \circ \zeta^{-1}\right)(0)$ and $\left(D^{j} f_{n}(0)\right)$ converges to $D^{j} f(0)$ for every $j \in\{1, \ldots, k\}$, Faa-di-Bruno's formula (30) shows that $\left(j_{\tilde{\zeta}\left(x_{n}\right)}^{k}\left(\tilde{\zeta} \circ \zeta^{-1} \circ f_{n}\right)\right)_{n}$ converges to $j_{0}^{k}\left(\tilde{\zeta} \circ \zeta^{-1} \circ f\right)$. It turns out that $\left(j_{\tilde{\zeta}\left(x_{n}\right)}^{k}\left(\tilde{\zeta}\left(S_{n} \cap \tilde{U}\right)\right)\right)_{n}$ converges to $j_{0}^{k}(\tilde{\zeta}(S \cap U))$.

\section{Appendix B}

\section{Proof of the continuity result (Corollary 1.21)}

This section contains a proof of the continuity result stated for the invariant $k$-jets associated to a smooth $\operatorname{RDS}\left(\varphi_{n}\right)_{n \in \mathbb{Z}}$ over $\theta$ on a $C^{\infty}$ Riemannian manifold $M$.

Let us recall that $\Phi(\omega, x)$ denotes the map $\operatorname{Exp}_{\varphi(\omega)(x)}^{-1} \circ \varphi(\omega) \circ \operatorname{Exp}_{x}$ that is well-defined on the $\delta(\omega, x)$-ball $B_{x}(0, \delta(\omega, x))$ of $T_{x} M$. For every $n \in \mathbb{N}$, there exists a positive measurable function $\delta_{n}$, such that for every $(\omega, x) \in \Omega \times M$, the map

$$
\Phi_{n}(\omega, x):=\operatorname{Exp}_{\varphi_{n}(\omega)(x)}^{-1} \circ \varphi_{n}(\omega) \circ \operatorname{Exp}_{x}
$$

is a $C^{\infty}$ diffeomorphism from $B_{x}\left(0, \delta_{n}(\omega, x)\right)$ onto its image and is equal to $\Phi\left(\tilde{\theta}^{n-1}(\omega, x)\right) \circ \cdots \circ \Phi(\omega, x)$. Let $\mathcal{W}_{n}(\omega, x)$ denote the $d$-dimensional submanifold $\operatorname{Exp}_{x}\left(E^{s}(\omega, x) \cap B_{x}\left(0, \delta_{n}(\omega, x)\right)\right)$ and let $\mathcal{U}_{n}(\omega, x)$ denote the image of $\mathcal{W}_{n}\left(\tilde{\theta}^{-n}(\omega, x)\right)$ by $\varphi_{n}\left(\theta^{-n}(\omega)\right)$. The proof of Corollary 1.21 proceeds in three steps. First, the application of Corollary 1.19 will give that $\left(j_{x}^{k}\left(\mathcal{U}_{n}(\omega, x)\right)\right)_{n}$ converges in probability to a $k$-jet, denoted by $\mathcal{S}_{1}^{(k)}(\omega, x)$, which is invariant by the RDS $\left(\varphi_{n}\right)$. Secondly, with the aid of the "local RDS on the bundle $T M$ " $\left(\Phi_{n}\right)$ over $\tilde{\theta}$, one shows that: 
- the $k$-jets of the manifolds $\tilde{\mathcal{U}}_{n}(\omega, x):=\operatorname{Exp}_{x}^{-1}\left(\mathcal{U}_{n}(\omega, x)\right)$ at 0 converge to the $k$-jet at 0 of a submanifold of $T_{x} M$ denoted by $\tilde{S}(\omega, x)$;

- if $\left(x_{n}\right)_{n}$ is a sequence in $\Lambda_{R, \epsilon}(\omega)$ that converges to $x$, then $\left(j_{0}^{k}\left(\tilde{S}\left(\omega, x_{n}\right)\right)\right)_{n}$ converges to $j_{0}^{k}(\tilde{S}(\omega, x))$.

Finally, one shows that the $k$-jet $\mathcal{S}^{(k)}(\omega, x):=j_{x}^{k}\left(\operatorname{Exp}_{x}(\tilde{S}(\omega, x))\right)$ is $\mu$-almost surely equal to $\mathcal{S}_{1}^{(k)}(\omega, x)$ and that, if $\left(x_{n}\right)_{n}$ is a sequence in $\Lambda_{R, \epsilon}(\omega)$ that converges to $x$, then $\left(\mathcal{S}^{(k)}\left(\omega, x_{n}\right)\right)_{n}$ converges to $\mathcal{S}^{(k)}(\omega, x)$.

First step. - Let $\Psi: T M \rightarrow M \times \mathbb{R}^{N}$ denote a bimeasurable bijection covering $\operatorname{id}_{M}$ such that for each $x \in M$, the restriction $\psi(x)$ of $\Psi$ to the fiber $T_{x} M$ is an isometry between $\left(T_{x} M,\|\cdot\|_{x}\right)$ and the Euclidean space $\left(\mathbb{R}^{N},|\cdot|\right)$. A construction of such a map is detailed in [1]. Let $\zeta(x)$ denote the map $\psi(x) \circ \operatorname{Exp}_{x}^{-1}$ for every $x \in M$. The map $\zeta$ belongs to $\mathcal{C}_{1}(M)$ and the map $\Phi_{\zeta}=\zeta \circ \varphi \circ \zeta^{-1}$ satisfies the assumptions of Corollary 1.19. It turns out that $\left(j_{x}^{k}\left(\mathcal{U}_{n}(\omega, x)\right)\right)_{n}$ converges in probability to a $k$-jet at $x$ denoted by $\mathcal{S}_{1}^{(k)}(\omega, x)$ and that $\mathcal{S}_{1}^{(k)}$ is invariant by the $\operatorname{RDS}\left(\varphi_{n}\right)_{n}$.

Second step. - Let $\tilde{\mathcal{U}}_{n}(\omega, x)$ denote the submanifold of $T_{x} M$ defined by $\operatorname{Exp}_{x}\left(\mathcal{U}_{n}(\omega, x)\right)=\Phi_{n}\left(\tilde{\theta}^{-n}(\omega, x)\right)\left(E^{s}\left(\tilde{\theta}^{-n}(\omega, x)\right) \cap B_{\varphi_{-n}(\omega)(x)}\left(0, \delta_{n}\left(\theta^{-n}(\omega)\right)\right)\right)$. The proof of the first assertion of Theorem 1.7 can be rewritten, word for word, by substituting $\Phi(\omega, x)$ for $\phi(\omega)$ and $\tilde{\theta}$ for $\theta$. This shows that $\left(j_{0}^{k}\left(\tilde{\mathcal{U}}_{n}\right)(\omega, x)\right)_{n}$ converges to the $k$-jet at 0 of a submanifold $\tilde{S}(\omega, x)$ of $T_{x} M$ for $\mu$-almost every $(\omega, x)$. More precisely, if $f_{n}$ denotes an orthogonal parametrization of $\tilde{\mathcal{U}}_{n}$ at 0 and if $\Pi$ denotes the orthogonal projection onto $E^{s}$, then for every $j \in\{1, \ldots, k\},\left(D^{j}\left(f_{n} \circ \Pi\right)(0)\right)_{n}$ converges $\mu$-almost surely to a map denoted by $\alpha_{j}$. The restriction $f$ of $\sum_{j=1}^{k} \alpha_{j} /(j !)$ to $E^{s}(\omega, x)$ is an orthogonal parametrization of $\tilde{S}(\omega, x)$ at 0 .

Let $\Omega_{k}$ denote the subset of points $\omega \in \Omega$ such that

$$
\sup _{n \in \mathbb{Z}, x \in M} \mathrm{e}^{-|n| \epsilon}\left\|D^{j} \Phi\left(\tilde{\theta}^{n}(\omega, x)\right)(0)\right\|_{\varphi_{n}(\omega)(x)}
$$

is finite for every $j \in\{2, \ldots, k\}$. By hypothesis, $\mathbb{P}\left(\Omega_{k}\right)=1$. Let $\epsilon$ be a positive number smaller than $\frac{1}{20} \lambda_{s}$ or $\frac{1}{20 k}\left(k \lambda_{s}-\lambda_{s+1}\right)$ depending on whether $\lambda_{s}>0$ or $\lambda_{s} \leq 0$. It follows from the definitions of $\Lambda_{R, \epsilon}$ and $\Omega_{k}$ that, for $\omega \in \Omega_{k}$, there exists a finite real $C(\omega)>0$ such that for every $x \in \Lambda_{R, \epsilon}(\omega)$

$$
\begin{aligned}
& \left\|D^{m} \Phi\left(\tilde{\theta}^{-j}(\omega, x)\right)(0)\right\|_{\varphi_{-j}(\omega)(x)} \leq C(\omega) \mathrm{e}^{j \epsilon} \quad \text { for } m \in\{2, \ldots, k\} \text { and } j \in \mathbb{N}, \\
& \left\|(\mathrm{I}-\Pi(\omega, x)) D \Phi_{j}\left(\tilde{\theta}^{-j}(\omega, x)\right)(0)\right\|_{\varphi_{-j}(\omega)(x)} \leq C(\omega) \mathrm{e}^{j\left(\lambda_{s+1}+3 \epsilon\right)} \quad \text { for } j \in \mathbb{N},
\end{aligned}
$$




$$
\begin{aligned}
& \left\|D \Phi_{-j}(\omega, x)(0) \Pi(\omega, x)\right\|_{x} \leq C(\omega) \mathrm{e}^{j\left(-\lambda_{s}+\epsilon\right)} \quad \text { for } j \in \mathbb{N}, \\
& \left\|D \Phi_{j-i}\left(\tilde{\theta}^{-j}(\omega, x)\right)(0)-D \Phi_{-i}(\omega, x)(0) \Pi(\omega, x) D \Phi_{j}\left(\tilde{\theta}^{-j}(\omega, x)\right)(0)\right\|_{\varphi_{-j}(\omega)(x)} \\
& \leq C(\omega) \mathrm{e}^{(j-i) \lambda_{s+1}+2(j+i) \epsilon}
\end{aligned}
$$

for $i, j \in \mathbb{N}^{*}$ such that $i \leq j$ and

$$
\left\|D \Phi_{-i}(\omega, x)(0) \Pi(\omega, x) D \Phi_{j}\left(\tilde{\theta}^{-j}(\omega, x)\right)(0)\right\|_{\varphi_{-j}(\omega)(x)} \leq C(\omega) \mathrm{e}^{(j-i) \lambda_{s}+3(i+j) \epsilon}
$$

for $i, j \in \mathbb{N}^{*}$ such that $j \leq i$.

These terms will replace, in this order, the maps $B^{(m)}\left(\theta^{-j}\right),(\mathrm{I}-\Pi) A_{j}\left(\theta^{-j}\right)$, $A_{-j} \Pi, \tilde{A}_{i, j}$ and $A_{-i} \Pi A_{j}\left(\theta^{-j}\right)$ of Section 3.2. By using these inequalities, instead of inequalities (16), (17), (18), (19) and (20) in Section 3.2, one obtains, instead of Proposition 3.8, that

$$
\left(\left\|D^{j}\left(f_{n}(\omega, x) \circ \Pi(\omega, x)\right)(0)-D^{j}(f(\omega, x) \circ \Pi(\omega, x))(0)\right\|_{x}\right)_{n}
$$

converges to 0 uniformly in $\Lambda_{R, \epsilon}(\omega)$, for each $\omega \in \Omega_{k}$ and $j \in\{2, \ldots, k\}$.

Consider a relatively compact chart $(\zeta, U)$ at a point $x \in \Lambda_{R, \epsilon}(\omega)$ in order to identify $T U$ with $\zeta(U) \times \mathbb{R}^{N}$ and let $f_{\zeta}$ denote the restriction of $T_{x} \zeta$ ० $f(\omega, x) \circ\left(T_{x} \zeta\right)^{-1}$ to a neighbourhood of 0 in $T_{x} \zeta\left(E^{s}(\omega, x)\right)\left(f_{\zeta}(\omega, x)\right.$ is a "parametrization" of $T_{x} \zeta(\tilde{S}(\omega, x))$ at 0$)$. The previous convergence result can be rewritten as follows: for each $\omega \in \Omega_{k}$ and $j \in\{2, \ldots, k\}$, the sequence $\left(D^{j}\left(T_{y} \zeta \circ f_{n}(\omega, y) \circ \Pi(\omega, y) \circ\left(T_{y} \zeta\right)^{-1}\right)(0)\right)_{n}$ converges uniformly for in $\Lambda_{R, \epsilon}(\omega) \cap U$ (with respect to the usual metric of $\mathbb{R}^{N}$ ). Assume furthermore that $\epsilon$ is smaller than $\left(\lambda_{i}-\lambda_{i+1}\right) / 4$ for every $i \in\{1, \ldots, r-1\}$. It follows from the continuity of $y \mapsto E^{s}(\omega, y)$ on $\Lambda_{R, \epsilon}(\omega)$ and the regularity of the maps Exp and $\varphi$ that the map $y \mapsto D^{j}\left(T_{y} \zeta \circ f_{n}(\omega, y) \circ \Pi(\omega, y) \circ\left(T_{y} \zeta\right)^{-1}\right)(0)$ is continuous at $x$ for every $j \in \mathbb{N}^{*}$ and $n \in \mathbb{N}$. Thus the map $y \mapsto D^{j} f_{\zeta}(\omega, y)(0)$ is continuous at $x$. This shows that, if $\left(x_{n}\right)$ is a sequence of points of $\Lambda_{R, \epsilon}(\omega)$ that converges to $x$, then $j_{0}^{k}\left(T_{x_{n}} \zeta\left(\tilde{S}\left(\omega, x_{n}\right)\right)\right)$ converges to $j_{0}^{k}\left(T_{x} \zeta(\tilde{S}(\omega, x))\right)$.

Third step. - Consider a point $(\omega, x) \in \Omega \times M$ such that the sequences $\left(j_{0}^{k}\left(\mathcal{U}_{n}(\omega, x)\right)\right)_{n}$ and $\left(j_{0}^{k}\left(\tilde{\mathcal{U}}_{n}(\omega, x)\right)\right)_{n}$ converge. Let $S(\omega, x)$ denote the submanifold of $M$ defined by $\operatorname{Exp}_{x}\left(\tilde{S}(\omega, x) \cap B_{x}(0, \delta(\omega, x))\right)$ and let $\mathcal{S}^{(k)}(\omega, x)$ denote its $k$-jet at 0 . Let us note that, if $(\zeta, U)$ is a chart at $x$, then

$$
j_{0}^{k}(\zeta(S(\omega, x)))=j_{0}^{k}\left(\zeta \circ \operatorname{Exp}_{x} \circ\left(T_{x} \zeta\right)^{-1} \circ T_{x} \zeta(\tilde{S}(\omega, x))\right) .
$$

It follows from the second step and the Faa-di-Bruno's formula that:

- The $k$-jet $j_{0}^{k}\left(\zeta \circ \operatorname{Exp}_{x} \circ\left(T_{x} \zeta\right)^{-1} \circ T_{x} \zeta\left(\mathcal{U}_{n}\right)\right)$ (which is equal to $j_{0}^{k}\left(\zeta\left(\mathcal{U}_{n}\right)\right)$ ) tends to $j_{0}^{k}(\zeta(S(\omega, x)))$ as $n$ tends to $+\infty$. This yields the equalilty $\mathcal{S}^{(k)}(\omega, x)=\mathcal{S}_{1}^{(k)}(\omega, x)$. 
- If $x \in \Lambda_{R, \epsilon}(\omega)$ and if $\left(x_{n}\right)$ is a sequence in $\Lambda_{R, \epsilon}(\omega)$ that converges to $x$, then the sequence $\left(j_{0}^{k}\left(\zeta \circ \operatorname{Exp}_{x_{n}} \circ\left(T_{x_{n}} \zeta\right)^{-1} \circ T_{x_{n}} \zeta\left(S\left(\omega, x_{n}\right)\right)\right)\right)_{n}$ converges to $j_{0}^{k}(\zeta(S(\omega, x)))$. Thus $\left(\mathcal{S}^{(k)}\left(\omega, x_{n}\right)\right)_{n}$ converges to $\mathcal{S}^{(k)}(\omega, x)$.

\section{BIBLIOGRAPHY}

[1] Arnold (L.) - Random dynamical systems, Springer, 1998.

[2] Cranston (M.) \& Le JAn (Y.) - Asymptotic curvature of random dynamical systems, in Stochastic Dynamics (Crauel (H.) \& Gundlach (M.), eds.), 1998.

[3] _ Geometric evolution under isotropic flow, Electronic Journal of Probability, t. 3 (1998), pp. 1-36.

[4] Kato (T.) - Perturbation theory for linear operators, 2nd ed., Springer, 1976.

[5] KIfER (Y.) - Ergodic theory of transformations, Progress in Probability and Statistics, vol. 10, Birkhäuser, 1986.

[6] Kingman (J.F.C.) - The ergodic theory of subadditive processes, J. Royal. Statist. Soc., t. B.30 (1968), pp. 499-510.

[7] Kunita (H.) - Stochastic flows and stochastic differential equations, Cambridge University Press, 1990.

[8] LE JAN (Y.) - Asymptotic properties of isotropic Brownian flows, in Spatial Stochastic Processes, Progress in Probability, Birkhäuser, 1991, pp. 219-232.

[9] _ A second order extension of Oseledets theorem, in Lyapunov exponents, Lect. Notes Math., vol. 1486, Springer, 1991, pp. 81-85.

[10] LEDRAPPIER (F.) - Quelques propriétés des exposants caractéristiques, in École d'été de Probabilité de Saint-Flour XII, Lect. Notes Math., vol. 1097, Springer, 1982, pp. 305-396.

[11] Oseledets (V.I.) - A multiplicative ergodic theorem. Lyapunov characteristic number for dynamical systems, Trans. Moscow Math. Soc., t. 19 (1968), pp. 197-231.

[12] Pesin (Y.B.) - Families of invariant manifolds corresponding to nonzero characteristic exponents, Math. USSR Izvestija, t. 10 (1976), pp. 12611305.

[13] - Characteristic Lyapunov exponents and smooth ergodic theory, Russian Math. Surveys, t. 32 (1977), pp. 55-114.

[14] Ruelle (D.) - Ergodic theory of differentiable dynamical systems, Publ. Math. I.H.E.S., t. 50 (1979), pp. 27-58.

[15] Characteristic exponents and invariant manifolds in Hilbert space, Annals of Math., t. 115 (1982), pp. 243-290. 